\title{
Far-field and Near-field Optical Properties Of Strongly Interacting Silver Nanocrystals
}

\author{
By
}

Daniel Prezgot

A thesis submitted to the Faculty of Graduate and Postdoctoral Affairs in partial fulfillment of the requirements for the degree of

\author{
Master of Science \\ in \\ Chemistry \\ Carleton University, \\ Ottawa, Ontario
}

(C) 2014

Daniel Prezgot 
Abstract

Noble metal nanocrystals are known for their remarkable optical properties that are caused by the result of their ability to support a localized surface plasmon resonance (LSPR). These optical properties can be observed in the far-field, through their optical extinction or in the near-field by a highly enhanced electric-field localised to the surface of the particles. The goal of this work was to study properties of supported silver nanocrystal ensembles as they interact with their substrate, neighbouring nanocubes and the resulting mutual interactions. Demonstrated is the ability to finely tune plasmonic properties of silver nanocube monolayers by controlling the interparticle spacing and the properties of the underlying substrate. Control over these properties is applied to the optimisation of substrates used for surface-enhanced Raman spectroscopy (SERS). Control over the plasmonic properties is achieved by directing the assembly of silver nanocube (AgNC) ensembles. The primary means to do so is by using the Langmuir-Blodgett technique to control nanoparticle surface density. These monolayers are placed on a number of substrates such as glass, silicon thin films, and titanium oxide thin films. Demonstrated in this work is the ability to shift plasmonic modes using interparticle interactions or particle-substrate interactions. The local electric field enhancement in these monolayers is investigated thoroughly by SERS, and demonstrated is the dependence of the enhancement on particle cluster size, charge transfer processes, and the location of the target molecule in the monolayer. By gaining insight into how these interactions affect the local electric field at the surface of the nanoparticles, lessons gained through this work could be applied towards the optimization of surface enhanced Raman spectroscopy. 


\section{Acknowledgements}

I would like to acknowledge my supervisor, Dr. Anatoli Ianoul, for providing me with the direction, inspiration, guidance, and comedy which has contributed to this work. I would like to thank him for his patience as I eat and sleep during time better spent in another manner. I would also like to acknowledge the rest of the Ianoul lab and its graduate students, Adam Bottomely, Devin O’Neill, Grace Idiong, Alyssa Staff, and Amy Won as well as the numerous lesser beings who have cycled through our group such as Iryna Buznytska, Vlad Pripotnev and Irving Rosas-Brugada for their assistance, comradery, and constant distraction.

I would like to acknowledge the assistance provided by Dr. Jianqun Wang and the Carleton University nano-imaging facility for the TEM characterization, as well as Rob Vandusen, Adam Bottomley and the Carleton University Microfabrication Facility for the fabrication of the thin-films used in this work.

I would like to acknowledge the personal funding provided by the Carleton University, the GSA, and Fluorsense Inc. Additionally I would like to acknowledge NSERC, CFI, and Spartan Bioscience for the funding provided to the Ianoul lab.

I would like to acknowledge my good friends Eric Watson and Cody Crosby, for being just that. Also a certain A.B. who has already received enough acknowledgement...

I would like to thank my parents, Zofia and Marek Prezgot, without their support my achievements to date would never have been possible.

And lastly my thanks go to the rest of the good people of the Carleton Chemistry department. I look forward to their continued company and education, though I dread continuing to walk the halls of Steacie. I can only hope that one day I will be free of them. 


\section{Table of Contents}

1 Theory of Plasmonics, Plasmonic Interactions, and the Synthesis and Assembly of Silver

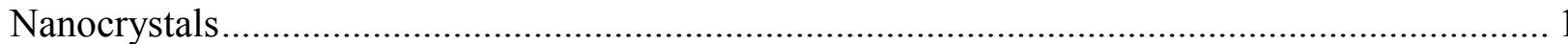

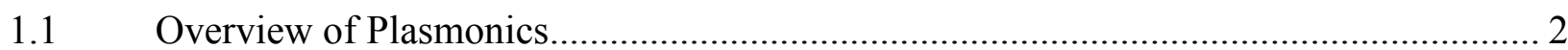

1.1.1 When can a LSPR occur? .......................................................................... 3

1.1.2 Sensitivity of LSPR to environment ..................................................................... 4

1.1.3 The effect of size and shape of the nanoparticle on the LSPR properties ................ 5

1.2 Coupling of Localized Surface Plasmon Resonances ...................................................... 8

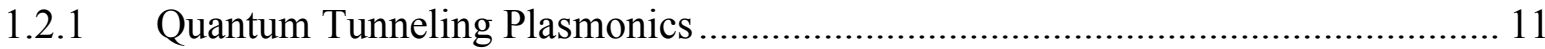

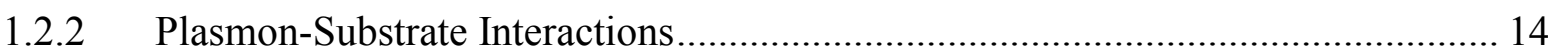

1.3 Synthesis of Silver nanostructures ………................................................................. 17

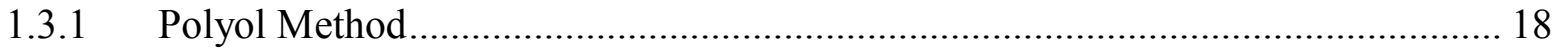

1.3.2 Reduction Mechanism of Ag in the Polyol Method ............................................. 18

1.3.3 Nucleation of Nanoparticles ................................................................................. 19

1.3.4 Shape Controlled Growth and the Function of the Capping Agent ........................ 21

1.3.5 Seed selection through Oxidative Etching ......................................................... 22

1.3.6 Kinetically Controlled Selection of Seeds .......................................................... 23

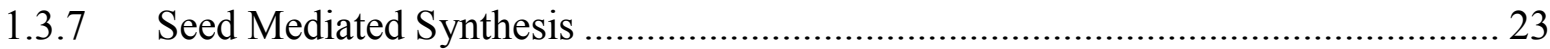

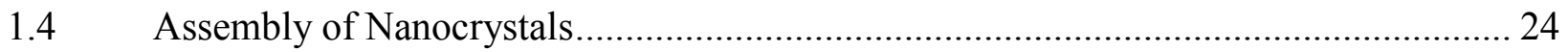

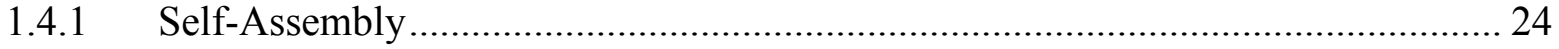

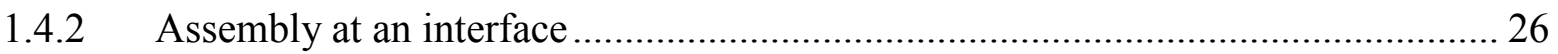

1.4.3 The Langmuir-Blodgett Technique.................................................................... 27

1.5 Surface Enhanced Raman Spectroscopy (SERS) ........................................................ 30

1.5.1 The SERS Enhancement Factor ……………………………………………..... 31

1.5.2 Electromagnetic (EM) Mechanism of SERS enhancement .................................. 34

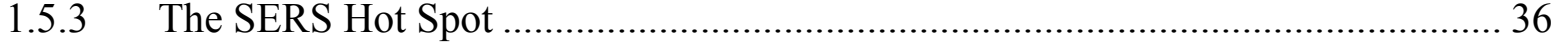

1.5.4 Non EM-Enhancement: Chemical Enhancement ………………………............. 39

1.5.5 Resonance Raman Effects................................................................................ 40

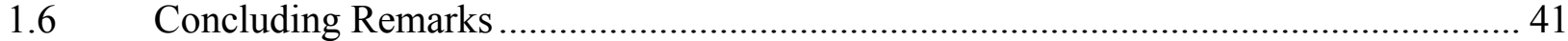

2 Strongly Interacting Silver Nanocubes on Silicon Substrates .............................................. 42

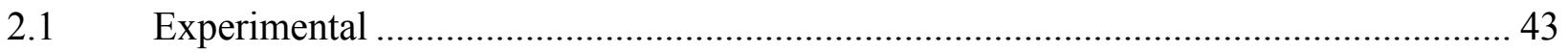




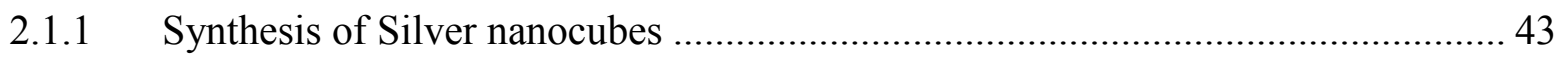

2.1.2 Langmuir - Blodgett Silver Nanocube Monolayers ………………………............ 44

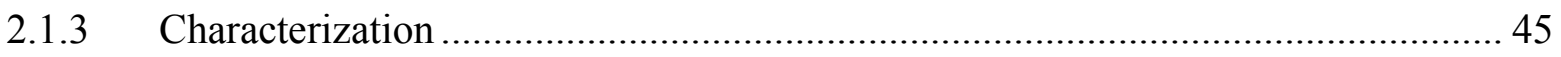

2.1.4 Surface - Enhanced Raman Spectroscopy ............................................................. 46

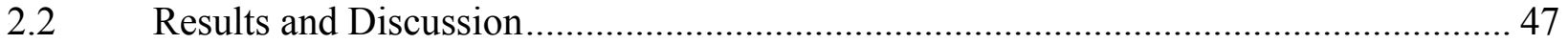

2.2.1 Optical Properties of Strongly Interacting Nanocubes on Silicon Substrates......... 47

2.2.2 Surface-Enhanced Raman Spectroscopy ………………………………….......... 51

2.2.3 Aging of the Silicon Substrates and Re-evaluation of Some Observations............ 55

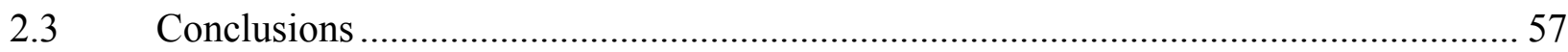

3 Lessons in the Assembly of Silver Nanocubes.................................................................. 58

3.1 Coalescence of Silver Nanocubes in Langmuir-Blodgett Monolayers ........................... 59

3.2 Surface Modification and Self-Assembly of Nanocubes by Alkanethiols...................... 62

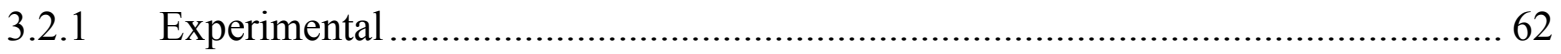

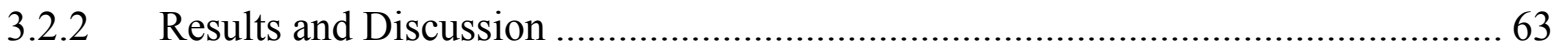

4 Interacting Silver Nanocubes on High-Refractive Index Oxide Substrates ............................. 66

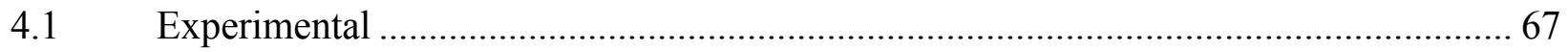

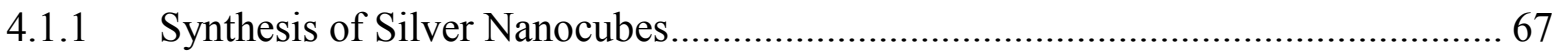

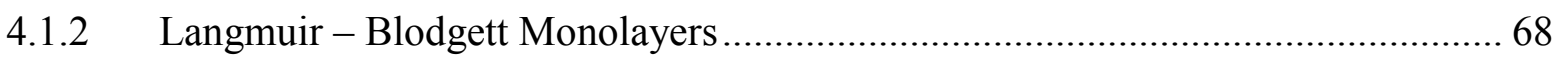

4.1.3 Post-modification of AgNC monolayers with benzenethiol (BT) .......................... 69

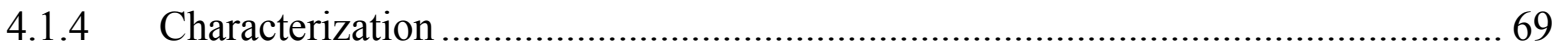

4.1.5 Surface-Enhanced Raman Spectroscopy …………………………………...... 70

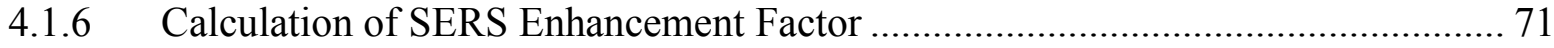

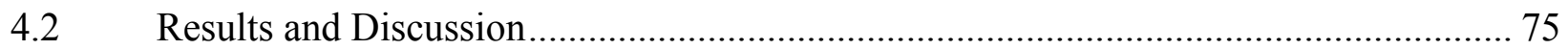

4.2.1 Optical properties of interacting silver nanocubes on Titanium Oxide .................. 75

4.2.2 Surface-Enhanced Raman Spectroscopy ........................................................... 76

4.2.3 Correlation between Near-field Enhancements with the Far-field Optics............... 82

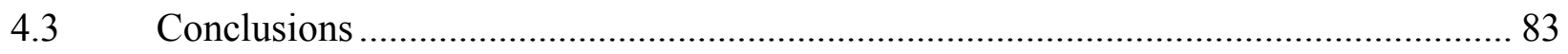

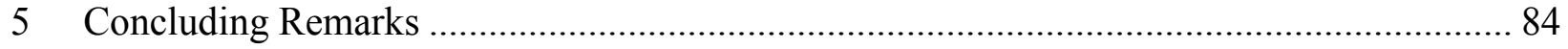

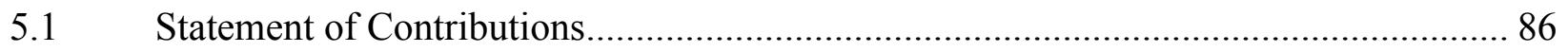




\section{Table of Figures}

Figure 1.1 - Schematic illustration of a dipolar localized surface plasmon resonance (LSPR) .... 2 Figure 1.2 - Schematic illustration of longitudinal (left) and transverse (right) coupling modes in a nanoparticle dimer.

Figure 1.3 - Plasmon hybridization scheme of an interacting nanoparticle dimer...................... 9 Figure 1.4 - Schematic illustration of the classical regime $\left(\mathrm{d}_{\mathrm{CR}}\right)$, quantum regime $\left(\mathrm{d}_{\mathrm{QR}}\right)$, and conductive contact regime $\left(\mathrm{d}_{\mathrm{CC}}\right)$.

Figure 1.5 - Displays the theoretical (a,d) plasmon energies, (b,e) far-field extinction crosssection, and (c,f) E-field enhancement in the gap between two small metal nanoparticles, modelled both classically and with a quantum-corrected model

Figure 1.6 - a) Plasmon hybridization scheme of a nanocube interacting with a dielectric substrate and electric field intensity of the hybridized modes

Figure 1.7 - Model describing the nucleation and growth of nanocrystals based on atomic

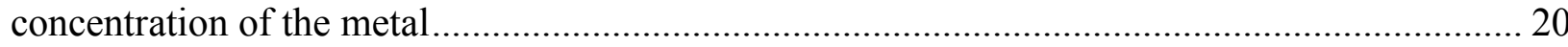

Figure 1.8 - Possible reaction paths in the polyol synthesis of Ag nanostructures ..................... 21 Figure 1.9 - Langmuir-Blodgett isotherm of a silver nanocube monolayer with TEM images of monolayers deposited at each analogue phase. 28

Figure 1.10 - Photographs of a silver-nanocube monolayer on dispersed at the air/water interface on a Langmuir-Blodgett trough

Figure 1.11 - Typical long-tail probability distribution of single-molecule enhancement factors

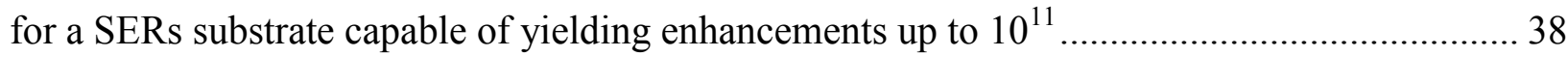

Figure 2.1 - Schematic of the Raman spectrometer used in this chapter. ................................... 46 Figure 2.2 - A) UV/Vis extinction spectrum of colloidal 40nms nanocubes suspended in ethanol and B) a transmission electron micrograph of an individual nanocube

Figure 2.3 - A) Atomic force microscopy low-pressure $(1 \mathrm{mN} / \mathrm{m})$ Langmuir-Blodgett monolayer of 40nm silver nanocubes deposited on glass B) TEM image of a similar monolayer of 30nm

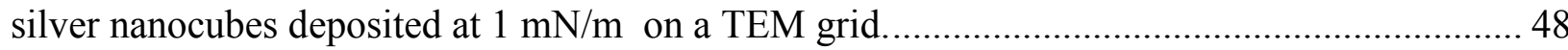

Figure 2.4 - A) UV/Visible extinction spectra on glass of silver nanocube monolayers deposited at various surface pressures. B) TEM image from a similar set of monolayers, exhibiting subnanometer junctions and conductive contact.

Figure 2.5 - A) UV/Visible spectroscopy of silver nanocube monolayers deposited on silicon at various surface pressures and B) UV/Visible spectroscopy of silver nanocube monolayers deposited on varying thicknesses of silicon.

Figure 2.6 - SERS Spectra of Rhodamine B and PVP obtained in this experiment with 458nm excitation

Figure 2.7 - Histograms of normalised average intensity of Raman scattering observed at each excitation wavelength..

Figure 2.8 - Comparison of the intensity of SERS on glass against on $25 \mathrm{~nm}$ silicon thin film and SERS Spectra recorded at $458 \mathrm{~nm}$ of a AgNC monolayer deposited at $4.5 \mathrm{mN} / \mathrm{m}$. 54 
Figure 2.9 - Silver nanocube monolayers deposited at $3 \mathrm{mN} / \mathrm{m}$ on varying thicknesses of Si after the slides had been aged in air for several months............................................................. 56

Figure 3.1 - Spontaneous coalescence of nanocube assemblies in Langmuir films.................. 59

Figure 3.2 - Coalescence and stability of different sized nanocubes. ................................... 61

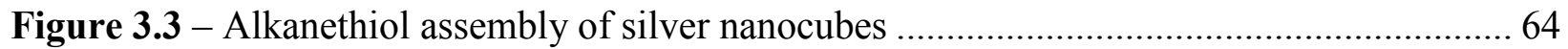

Figure 4.1 - Schematic diagram of the Raman Microscopy setup used in this chapter ............. 70

Figure 4.2 - UV/Visible extinction spectra for a monolayer deposited at $6 \mathrm{mN} / \mathrm{m}$ on varying

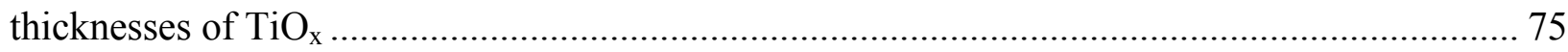

Figure 4.3 - A - C) TEM images of monolayers produced at 1.5, 6, and $15 \mathrm{mN} / \mathrm{m}$. D) UV-Vis spectra of aforementioned monolayers on Glass and $\mathrm{E}$ ) on a $10 \mathrm{~nm} \mathrm{TiO}_{\mathrm{x}}$ film ...................... 76 Figure 4.4 - A) Schematic showing the design of the monolayer. B) SERS Enhancement Factor as a function of wavelength for both Raman reporters; DPPE-RhB and Benzenethiol (BT), C) and D) SERS spectra collected at 488nm are displayed for both RhB and BT...................... 77 Figure 4.5 - UV/Vis extinction spectra of the monolayers deposited at $1.5 \mathrm{mN} / \mathrm{m}$, overlayed with DPPE-RhB SERS Enhancement Factors (points), excitation lines (458nm, 488nm, 514nm, $568 \mathrm{~nm})$, and a sample Raman spectrum collected at each excitation wavelength .................... 78 Figure 4.6 - UV/Vis extinction spectra of the monolayers deposited at $1.5 \mathrm{mN} / \mathrm{m}$, overlayed with BT SERS Enhancement Factors (points), excitation lines (458nm, 488nm, 514nm, 568nm, $647 \mathrm{~nm}$ ) and a sample Raman spectrum collected at each excitation wavelength

Figure 4.7 - A - D) TEM images of monolayers produced at $1.5 \mathrm{mN} / \mathrm{m}, 4 \mathrm{mN} / \mathrm{m}, 8 \mathrm{mN} / \mathrm{m}$, and $13 \mathrm{mN} / \mathrm{m}$. E) and F) SERS EF as a function of particle density for $514 \mathrm{~nm}$ and $568 \mathrm{~nm}$ excitation wavelengths

Figure 4.8 - Transmission, reflection, and absorption spectra of silver nanocube monolayers on $10 \mathrm{~nm}^{\mathrm{TiO}_{x}}$ 


\section{List of Abbreviations and Symbols}

LSPR: Localized surface plasmon resonance

SERS: Surface-Enhanced Raman Spectroscopy

SPP: Surface Plasmon Polariton

AgNP: Silver Nanoparticle

AgNC: Silver Nanocube

$\lambda$ : Wavelength

$\omega:$ Frequency

\&: Dielectric constant

$\alpha$ : Polarizability

RIS: Refractive index sensitivity

FOM: Figure of Merit

$\boldsymbol{E}$ : Electric Field

D: Dipolar mode

Q: Quadrupolar mode

BDP: Bonding Dipolar Mode

D-D: Dipole-dipole coupling mode (same as BDP)

CTP: Charge transfer plasmon

EG: Ethylene Glycol

PVP: Polyvinylpyrrolidone

LB: Langmuir-Blodgett

SERS EF: SERS Enhancement Factor

$\frac{d \sigma_{j}}{d \Omega}, \boldsymbol{\beta}$ : Differential Raman cross section

Rh6G: Rhodamine 6G

RhB: Rhodamine B

DOPC: dioleoyl phosphocholine

DPPE: dipalmitoyl phosphoethanolamine

DPPE-RhB: dipalmitoyl phosphoethanolamine - Rhodamine B

PVD: Physical Vapour Deposition

AFM: Atomic Force Microscopy

TEM: Transmission Electron Microscopy

HDT: Hexadecanethiol

DDT: Dodecanethiol

PT: Propanethiol

BT: Benzenethiol 


\section{Theory of Plasmonics, Plasmonic Interactions, and the Synthesis and Assembly of Silver Nanocrystals}

The following section will provide an extensive review into the properties of localised surface plasmon resonances (LSPRs) and how they interact with their environment and with each other. The study of such interactions is the focus of this work. Specifically the work will focus on the coupling of silver nanocubes, and how their near-field and far-field optical properties can be adjusted by their surrounding environment. The far-field optical properties refer to the resonances that can be established with incident electromagnetic radiation, particularly visible light in this case, while the near-field refers to the localised electric field enhancement exhibited by these nanocrystals. This field enhancement is of fundamental importance to a number of applications, though the focus will primarily on surface-enhanced Raman spectroscopy (SERS).

As such the theory behind the synthesis and assembly of silver nanocrystals is introduced. A number of techniques have developed which allow precise control over the size and shape of colloidal silver nanocrystals. Specifically the focus will be on the use of the silver nanocube, a simple reproducible geometry which interacts with its environment in unique ways. A description is given for assembly strategies of silver nanocrystals, as we attempt to control their properties and observe unique phenomena through their assembly.

SERS is not only an application that has arisen as the result of such properties, but also a tool that can be used to study them. Thus in this work is described the origin of SERS and the SERS effect, so that we may understand how SERS can be used to study plasmonic assemblies. 


\subsection{Overview of Plasmonics}

Surface plasmons are unique waves that propagate along the surface of a conductor, usually metals. These waves are the result of the interaction between free electrons of a conductor and incident light. In this interaction, the electrons in the conduction band of a metal oscillate collectively in resonance with the electric field component of the light as a response to the force exerted by it. This produces a wave confined to the surface of the material, formally referred to as a surface plasmon polariton (SPP). ${ }^{1}$

When the dimensions of a nanostructure are smaller than the wavelength of incident light, such as in a nanoparticle, the plasmon oscillates locally around the entire particle and it is termed a localised surface plasmon. The displacement of the finite number of electrons in the particle cause charge separation, which experience a restoring force creating an oscillation with a native frequency, which when in resonance with incident light results in a maximum in optical extinction. ${ }^{2}$ The charge separation induced by this localised surface plasmon resonance (LSPR) generates an electric field at the surface which is enhanced relative to the incident field as displayed in Figure 1.1. ${ }^{2,3}$

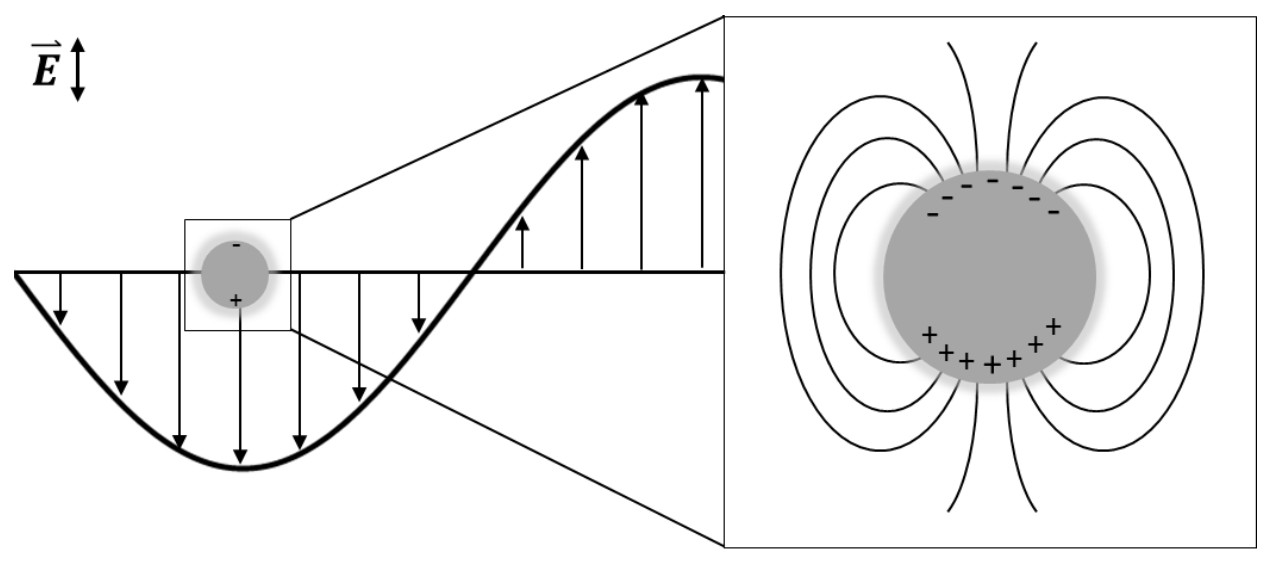

Figure 1.1 - Schematic illustration of a dipolar localized surface plasmon resonance (LSPR). Displacement of the conducting electrons by the electric field component of incident EM radiation produces a resonance which results in optical extinction, as well as an electric field localised to the surface of the nanoparticle which is enhanced relative to the incident field. 


\subsubsection{When can a LSPR occur?}

The Mie theory is a set of solutions to Maxwell's equations that describe the scattering of electromagnetic radiation by a sphere and can be used to describe the extinction cross section of a metal sphere as follows:

$$
C_{e x t}=\frac{24 \pi^{2} \alpha^{3} \varepsilon_{m}^{3 / 2}}{\lambda}\left[\frac{\varepsilon_{i}}{\left(\varepsilon_{r}+2 \varepsilon_{m}\right)^{2}+\varepsilon_{i}{ }^{2}}\right]
$$

where $a$ is the polarizability of the metal, $\lambda$ is the wavelength of incident light, $\varepsilon_{m}$ is the dielectric constant of the medium, and $\varepsilon_{r}$ and $\varepsilon_{i}$ are the real and imaginary components of the wavelength dependent dielectric function $\left(\varepsilon(\lambda)=\varepsilon_{r}(\lambda)+i \varepsilon_{i}(\lambda)\right)$ of the material of the nanoparticle. The real component describes the polarizability of the material while the imaginary component describes loss and absorption by the metal. From the above equation we can see that the resonance condition (maximum $C_{\text {ext }}$ ) is met when $\varepsilon_{r} \approx-2 \varepsilon_{m}$, and $\varepsilon_{i}$ is small. Note that the factor of 2 in front of $\varepsilon_{m}$ in the denominator is specific to a sphere, and may differ with other particle shapes. ${ }^{4}$ In general, it has been shown a high quality plasmon resonance arises when the magnitude of $\varepsilon_{r}$ is large and $\varepsilon_{i}$ is small. This qualitatively explains why Ag and Au are known to have strong surface plasmon resonances in the visible region. Ag displays a negative $\varepsilon_{\mathrm{r}}$ for wavelengths greater than $\sim 320 \mathrm{~nm}$ whereas Au displays such above $\sim 480 \mathrm{~nm}$. Ag steadily has a low $\varepsilon_{i}$ at wavelengths longer than $\sim 320 \mathrm{~nm}$, however Au shows a broad transition between high $\varepsilon_{i}$ and low $\varepsilon_{i}$ between $450-650 \mathrm{~nm}$, and begins increasing again past $750 \mathrm{~nm}$. This results in broader resonances and lower extinction efficiency in Au with respect to Ag. Thus Ag has the highest quality plasmons in the visible region of all metals, making it the ideal material for visible plasmonics. ${ }^{5}$ The drawbacks to using $\mathrm{Ag}$ as opposed to $\mathrm{Au}$ however is its increased 
susceptibility to oxidation in comparison to $\mathrm{Au}$, and its lack of biocompatibility, a property of $\mathrm{Au}$ which makes it ideal for biomedical applications. ${ }^{6}$

\subsubsection{Sensitivity of LSPR to environment}

Both SPs and LSPRs have been shown to be highly sensitive to the refractive index of their surrounding environment, leading to their prominent use in sensing applications.

The natural oscillating frequency of free electrons, or the plasma frequency, noting that by definition a plasmon is the quantization of a plasma wave, can be described by: ${ }^{2}$

$$
\omega_{p}=\sqrt{\frac{n e^{2}}{m \varepsilon_{0}}}
$$

where $\varepsilon_{0}$ is the permittivity of free space, $n$ is the electron density, and $e$ and $m$ are the effective charge and mass of an electron respectively. With this description a basic approximation of a surface plasmon frequency can be derived from the form of $\varepsilon_{r}$ from the Drude Model:

$$
\varepsilon_{r}=1-\frac{\omega_{p}^{2}}{\omega^{2}+\gamma^{2}}
$$

Where $\omega_{\mathrm{p}}$ is the bulk plasma frequency as described in equation (1), $\omega$ is the frequency of incident radiation, and $\gamma$ is the damping parameter. At visible and near-IR frequencies $\gamma \ll \omega$, and using the resonance condition, $\varepsilon_{\mathrm{r}}=-2 \varepsilon_{\mathrm{m}}$, this dependency can be described by: ${ }^{7}$

$$
\omega_{\max }=\frac{\omega_{p}}{\sqrt{2 \varepsilon_{m}+1}}
$$

Where $\omega_{\max }$ is the peak LSPR frequency, $\omega_{p}$ is the bulk plasmon frequency, and $\varepsilon_{m}$ is the dielectric constant of the medium. More conveniently expressed in terms of wavelength $(\lambda)$ and refractive index of the medium $\left(\varepsilon_{m}=n_{m}{ }^{2}\right)$ 


$$
\lambda_{\max }=\lambda_{p} \sqrt{2 n_{m}^{2}+1}
$$

This predicts a redshift in the plasmon peak position with increasing refractive index of the environment. While the relationship between $\lambda_{\max }$ and $n_{m}$ is not strictly linear, under small ranges of $n_{m}$ encountered in practical experiments it is approximately linear.

The sensing ability of any plasmonic mode can be evaluated by the magnitude of its red shift in comparison to the refractive index change of the environment. Therefore the refractive index sensitivity, RIS, of a LSPR is usually reported as peak shift per refractive index unit (nm/RIU):

$$
R I S=\frac{\mathrm{d} \lambda}{\mathrm{d} n}
$$

The precision to which the spectral shift can be observed depends on the width of the peak in question. It is thus popular to evaluate the sensing capability of using a Figure of Merit (FOM), which divides the RIS by the spectral line-width $(\Delta \lambda)$ or full width at half maximum of the peak: ${ }^{8}$

$$
F O M=\frac{R I S}{\Delta \lambda}
$$

\subsubsection{The effect of size and shape of the nanoparticle on the LSPR properties}

It should be noted that equations $1-5$ are derived from simple approximations which only hold true for ideal small metallic spheres $(<10 \mathrm{~nm})$. The Drude model from which they are derived do not consider the natural frequency of the oscillator, ignoring the restoring force which

dampens and red-shifts the resonant frequency of plasmon in larger nanostructures. ${ }^{9}$ Altering the shape of a nanoparticle will yield additional plasmonic modes to the simple dipolar resonance 
that has been discussed so far. ${ }^{10}$ Additionally, shaped nanoparticles have unique near-field enhancement properties which are of particular interest in sensing applications. Shape-controlled synthesis of metallic nanostructures has become a well-developed science ${ }^{10,11}$ and exploiting the unique properties that arise from altering the dimensions of nanocrystals can be of great benefit to the potential application of plasmonic nanostructures.

When increasing the size of a spherical nanoparticle the first observable effect is the redshifting and broadening of LSPR modes with increasing particle size. ${ }^{12}$ As particle size increases in relation to the incident wavelength, the electron cloud experiences increasing depolarization, damping the electron oscillation to lower energies. ${ }^{13}$ It is thus possible to tune LSPR position by control of a particle's size. The next observable effect is the appearance of higher order multipolar plasmonic modes. In small spherical nanoparticles $(<30 \mathrm{~nm})$ distribution of surface charges is homogeneous yielding only one possible plasmon resonance, a dipolar mode with angular momentum $l=1 .{ }^{12,14}$. As the size of the particle increases, multipolar charge distributions can manifest new plasmonic resonances. Of most importance is the second order $(l$ =2) quadrupolar mode, which becomes a significant feature in the extinction spectra of larger particles. ${ }^{15}$ Yet even higher order modes can exist, though highly dependent on the ability of the shape of the nanoparticle to support these multipolar resonances. Higher order LSPRs are always located at smaller wavelengths than the dipolar mode, which is further red-shifted by the presence of the high order electric field distributions. ${ }^{12}$ It is important to note that higher order modes may not necessarily display a net dipole moment, and may not resonate with incident light. These so-called "dark modes" are still of importance as their charge distribution can heavily influence the near-field properties of the nanostructures. ${ }^{16,17}$ 
Shape also plays an important role in the extinction properties of nanoparticles. The existence of sharp edges and corners facilitates the existence of higher order modes, even in smaller particles. ${ }^{12}$ In shaped particles charges are not distributed homogenously at the surface but rather are accumulated at the edges and corners, such that extra multipolar charges are induced. As such shaped particles, such as cubes or octahedrons, have clear, distinct identifiable extinction spectra characterised by the presence of high order modes at unique spectral positions. For example, silver nanocubes display a distinct octupolar resonance at $\sim 350 \mathrm{~nm} .^{18,19}$ The width and position of these higher order modes are indicative of the sharpness or conversely amount of truncation at the corners and edges. ${ }^{12}$ For a given plasmon mode, truncated particles exhibit a broader, higher energy resonance than its sharp-edged counterpart.

Elongated nanoparticles, such as ellipsoids, rods, or wires, possess plasmon resonances that are split between longitudinal and transverse modes. The position of the transverse modes are characteristic of the structures width and cross-sectional shape, while the longitudinal modes can be tuned by altering the nanocrystal's length. ${ }^{12}$ Thus elongated structures, such as nanorods, are particularly attractive in applications where controlling the position of the resonance is important, particularly in the red and near - IR regions of the spectrum, where these resonances are likely to reside for Ag and Au nanostructures.

An important consequence of the inhomogeneous charge distribution in shaped metal nanoparticles is the effect on their near-field electromagnetic enhancement properties. Charge accumulation at the edges and corners of nanoparticles with sharp features result in areas of exceptionally high electric field intensity. ${ }^{14,20}$ Geometries such as cubes, polyhedrons, and triangular plates are highly desired in field-enhanced applications such as surface enhanced Raman spectroscopy due to their capability to exhibit strong local electric field enhancement. ${ }^{21-}$ 
24 This also results in shaped particles exhibiting exceptionally high local refractive index sensitivity in comparison to spherical particles. $^{2}$

\subsection{Coupling of Localized Surface Plasmon Resonances}

Colloidal chemists have long noted drastic colour changes associated with the aggregation of nanoparticles. ${ }^{25,26}$ The far-field and near-field optical properties of aggregates or pairs of nanoparticles differ greatly from their individual counterparts. The main observable difference is the appearance of a red-shifted LSPR in coupled nanoparticles. This results in dramatic colour changes of the colloid, in the case of gold nanoparticles a clear red to blue colour transition is observed. ${ }^{27}$ This phenomenon has been used effectively in various bio-sensing assays where this dramatic and easily observable colour change allows for the detection of trace amounts of chemicals or biomolecules in solution. ${ }^{28}$ While these colour changes can be used in sensing applications and plasmonic rulers, ${ }^{29}$ strongly coupled nanoparticles find their most prominent use in field-enhanced applications such as SERS due to the high electric field intensity produced at the junctions. ${ }^{30}$

The simplest description of this interaction is the coupling of two nearby oscillating dipoles to form a new resonant frequency. Depending on the orientation of the incident electric field relative to the particles, the creation of transverse or longitudinal coupling modes relative to the dimer axis are possible. These dipoles may interact in either a parallel or anti-parallel fashion, however only the parallel oscillations will exhibit a dipole moment, and constitutes a "bright mode" which will interact with incident light. ${ }^{31}$ Conversely the anti-parallel modes are referred to as "dark modes" as they are not observable in the optical extinction spectra. The bright transverse mode will be slightly blue-shifted of the original dipole, while the longitudinal mode will be heavily red-shifted. These modes are illustrated in Figure 1.2, though note that the 
interaction of the transverse mode is weak, and does not yield further electric field enhancement, thus future discussion will be focused on the longitudinal coupling mode.

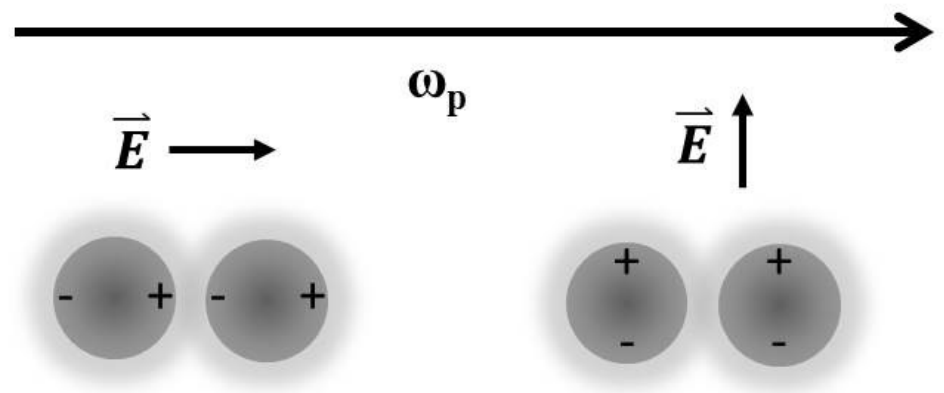

Figure 1.2 - Schematic illustration of longitudinal (left) and transverse (right) coupling modes in a nanoparticle dimer

A popular theoretical model which can be used to describe the interaction of plasmons is the plasmon hybridisation theory put forth by Nordlander et al. ${ }^{32}$ This model describes the mixing of interacting plasmon modes to form a new, hybridized mode. This simple model is essentially the electromagnetic analogue of molecular orbital theory, and can be used to intuitively predict or describe plasmonic interactions. ${ }^{33}$

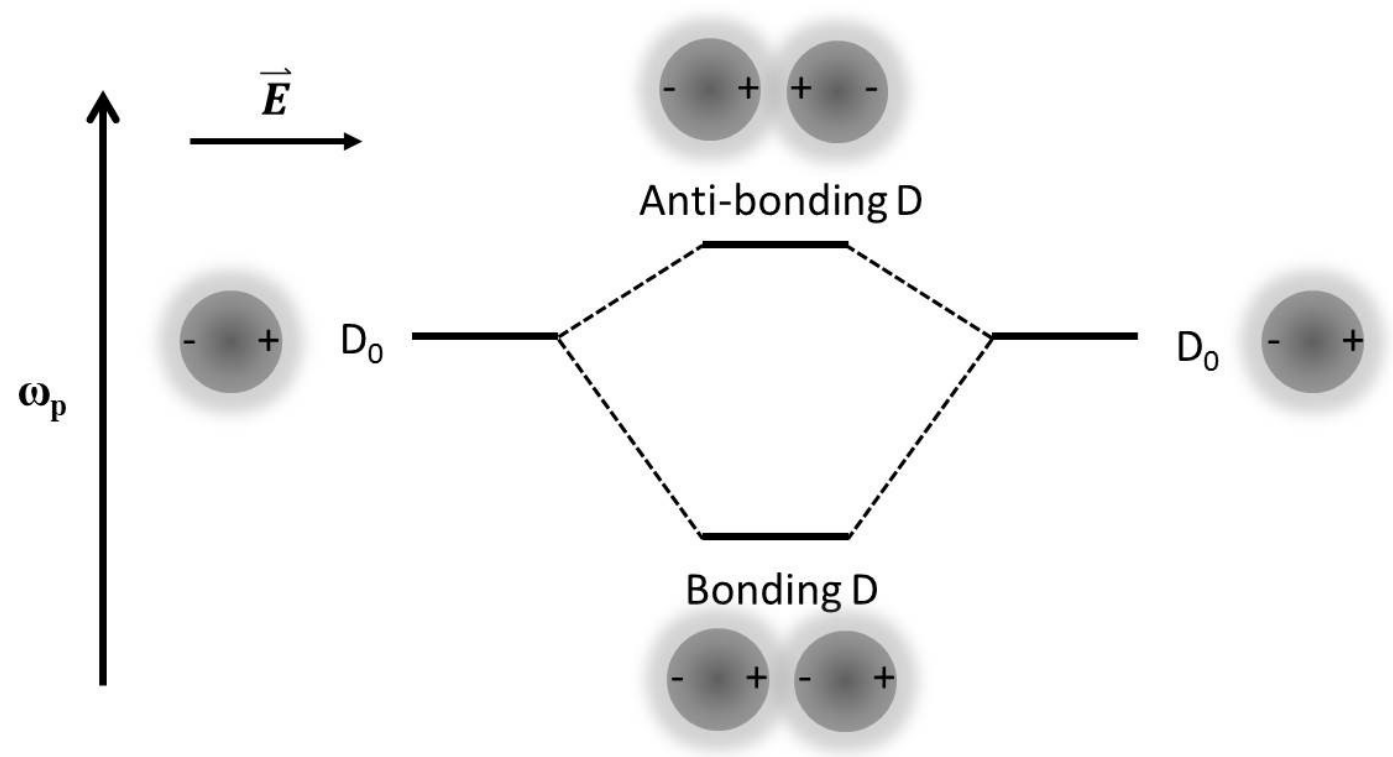

Figure 1.3 - Plasmon hybridization scheme of dipolar modes of an interacting nanoparticle dimer. Two interacting modes will mix to form an asymmetrically coupled anti-bonding mode and symmetrically coupled bonding mode. The anti-bonding mode will display no net dipole moment and will not resonant with incident radiation whereas the bonding mode will produce a strong resonance red-shifted of the original dipolar mode. 
Figure 1.3 shows a plasmon hybridisation scheme between two dipolar plasmonic modes in a metal nanoparticle. These modes will mix to form a high energy anti-bonding mode, and lower energy bonding mode. However the anti-bonding mode has virtually no net dipole moment, and is not easily excited by incident light, and constitutes a dark plasmon. The bonding mode is a bright plasmon however, and will result in a strong visible LSPR mode red-shifted of the original dipole. The energies of these modes are distance dependent, the degree of splitting increases as the interaction increases, proportional to $1 / \mathrm{d}^{3}$. The splitting is asymmetric however, with the bonding mode red-shifting at a much faster rate than the blue-shift of the anti-bonding mode. $^{32}$ The bonding dipolar mode (BDP) is thus very sensitive to interparticle distance, and its spectral position relative to the non-interacting dipole is indicative of the strength of the interaction between adjacent nanoparticles.

A consequence of notable importance in the coupling of two nanoparticles is the extraordinarily large electromagnetic enhancement located between the interacting pair. For the coupled oscillations where incident light is parallel to the interparticle access as has been discussed so far, charge density in the junction becomes very high. Opposing charges in the interparticle gap are allowed to get arbitrarily close, thus any point in the gap experiences a correspondingly large electric field. ${ }^{34}$ The field intensity experienced in these junctions tends to be $2-3$ orders of magnitude higher than that at the surface of an individual nanoparticle. ${ }^{30}$ 


\subsubsection{Quantum Tunneling Plasmonics}

According to the previous description, one would expect the interaction energy of plasmons in a nanoparticle dimer to increase exponentially with decreasing distance. ${ }^{32}$ What would be observed is the ever increasing red-shift in the bonding dipolar plasmon as the nanoparticles approach each other, until they are in direct contact. ${ }^{35,36}$ Once conductive overlap is established, a new charge transfer plasmon appears where conduction electrons are able to flow back and forth between the nanoparticle pair, acting as a single plasmonic mode. ${ }^{37}$ This mode replaces the bonding dipolar mode and originally appears near its position then subsequently blue shifts with increasing conductive overlap. The appearance of additional charge transfer modes may also be observed. ${ }^{38}$

Classical predictions describe an abrupt, discontinuous transition between the bonding dipolar (BDP) and charge transfer plasmon (CTP) modes, with an associated sudden shift in the plasmon resonance and reversal of the distance-dependent trend in plasmon shift (right column in Figure 1.5). ${ }^{39}$ There is a clear conceptual gap in this matter when using a classical description alone. The classical description breaks down at gap sizes less than $1 \mathrm{~nm}$ and does not coincide with experimental observations. ${ }^{38}$ In this regime it can become possible for electrons to tunnel between adjacent nanoparticles. Additionally, finite electron density in the interparticle cavity may screen plasmonic interactions responsible for the strong redshift observed in coupled nanostructures. ${ }^{40}$ Due to these effects, the bonding dipolar plasmon does not redshift to the degree predicted by classical theory, and the onset of the charge transfer plasmon occurs before conductive contact can be made, at gap distances of about $0.5 \mathrm{~nm}$ or less..$^{38,40,41}$ 


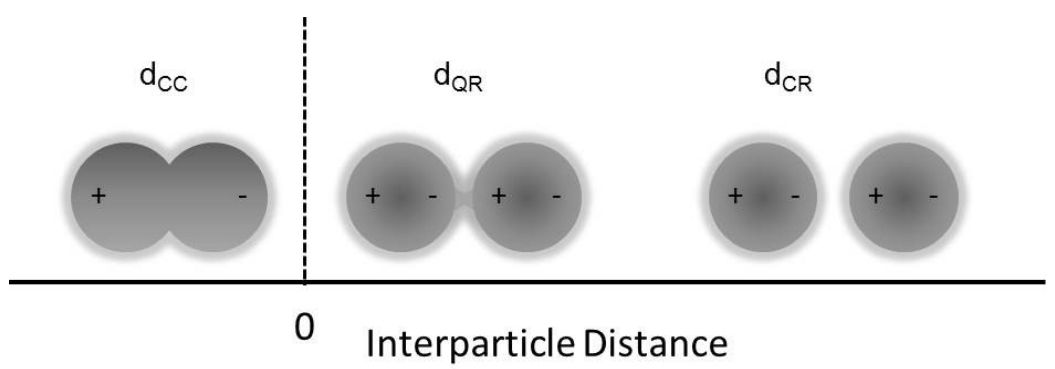

Figure 1.4 - Schematic illustration of the classical regime $\left(\mathrm{d}_{\mathrm{CR}}\right)$, quantum regime $\left(\mathrm{d}_{\mathrm{QR}}\right)$, and conductive contact regime $\left(\mathrm{d}_{\mathrm{CC}}\right)$

The onset of electron tunneling and the associated observations occur gradually as the junction between plasmonic structures reduces in size. Three distinct regimes exist as nanoparticles are placed closer together, illustrated in Figure 1.4, the classical or non-contact regime, the quantum tunneling regime, and the conductive contact regime. The precise distances at which these regimes occur depend on the plasmonic material, size, geometry and dielectric environment, however they will be described for typical distances calculated and observed for small silver or gold nanoparticles. ${ }^{40,38,42}$ At distances greater than $5 \AA$, no significant electron transfer can occur and this system can be modeled with classical electrodynamics. The bonding dipolar mode redshifts monotonically, and the localized electric field enhancement increases with reduced gap distance. ${ }^{43,44}$ At distances less than $5 \AA$ the tunneling probability of electrons begins to become significant. Initially, the bonding dipolar mode no longer shifts monotonically as the particles are brought closer together. As the distance closes to less than $\sim 2 \AA$ the potential barrier drops below the Fermi level allowing electrons to freely flow between nanoparticles. Electron density can now exist in the junction, replacing the bonding plasmons with charge transfer plasmons, which blue-shift with decreasing interparticle distance. This constitutes the charge transfer regime. Charge transfer plasmons are plasmonic modes that span both particles but behave as if they are a single, elongated non-spherical particle. ${ }^{42}$ The energies and optical extinctions of the BDP - CTP transition can be observed in Figure 1.5 (a-c). 

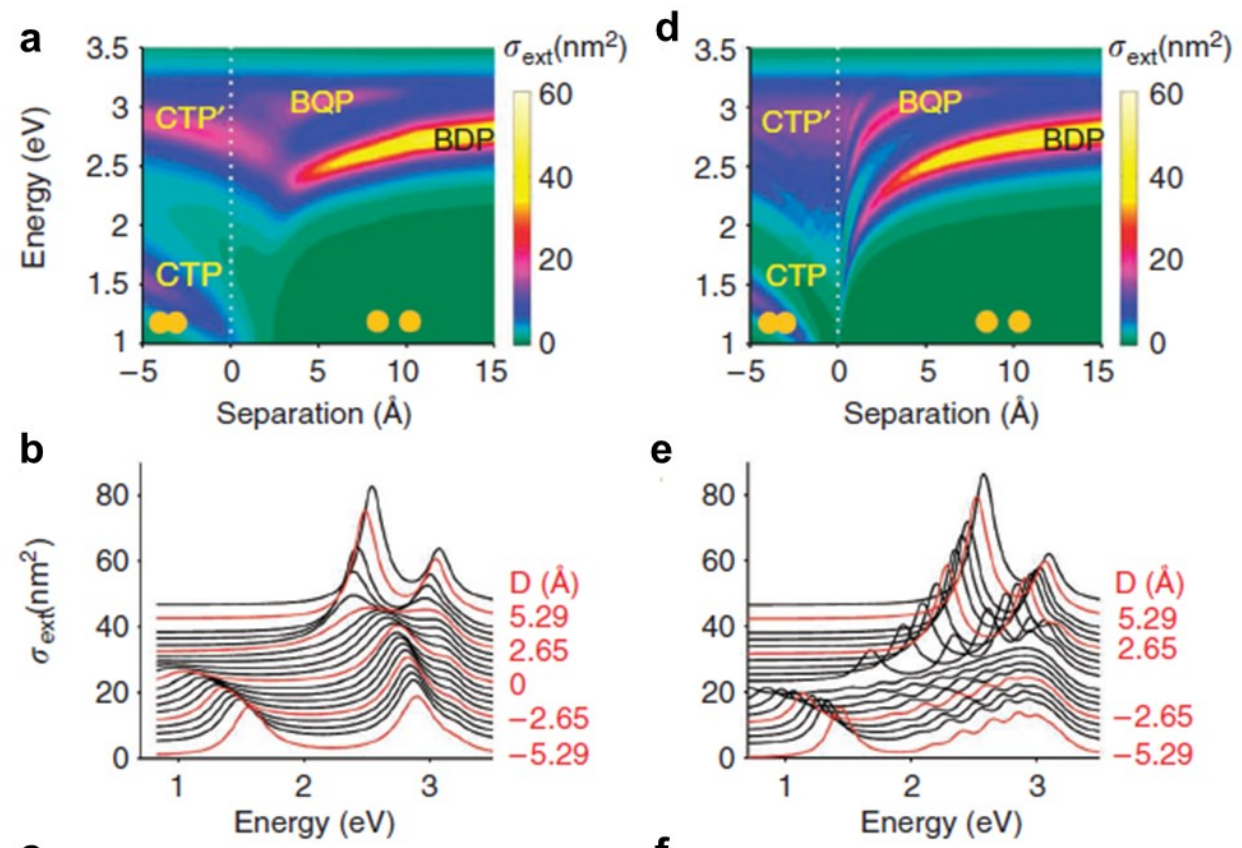

e
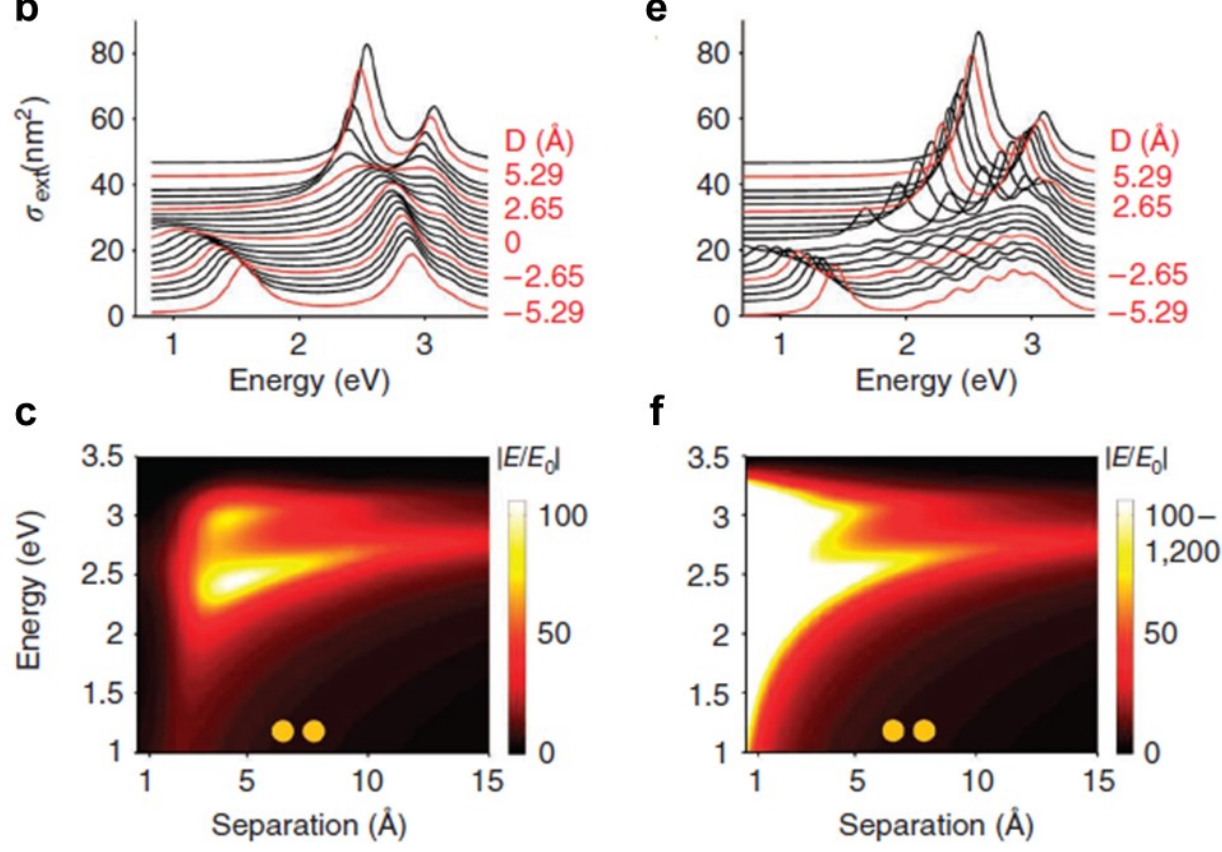

Figure 1.5 - Displays the theoretical (a,d) plasmon energies, (b,e) far-field extinction cross-section, and (c,f) E-field enhancement in the gap between two $2.17 \mathrm{~nm} \mathrm{Na}$ nanoparticles separated by distance R. (a-d) is modelled with a quantum corrected model, while (d-f) is modelled classically. Classical models predict an abrupt, discontinuous transition between bonding and charge-transfer plasmon modes, whereas the quantum corrected model displays a more gradual shift in plasmon energies in the quantum regime. Additionally E-field enhancement in the junction decreases significantly in the quantum regime at very small particle separations. Reproduced with permission from $\left({ }^{39}\right)$

The electric field enhancement in the nanoparticle junction is likewise affected by the tunneling of electrons between them as displayed in the bottom row of Figure 1.5. Classical models predict the electric field enhancement is increased as the gap between nanoparticles becomes smaller. ${ }^{39,40,44}$ Classical models, however, cannot correctly model field enhancements in the quantum tunneling regime. The onset of electron transfer neutralizes the high charge densities on opposite sides of the junction, resulting in drastic decreases in field enhancement. ${ }^{40,41}$ This is predicted to result in a severe drop in SERS intensity. ${ }^{45}$ 


\subsubsection{Plasmon-Substrate Interactions}

For many applications, plasmonic nanoparticles are required to be supported by a solid substrate. The sensitivity a nanoparticle's LSPR to its environment provides a unique and important interaction with the substrate which can be taken advantage of in sensing applications. The presence of a substrate breaks the symmetry of the nanoparticle's environment which has a profound effect on the plasmonic modes present. ${ }^{18,46}$

A metallic nanoparticle close to a substrate will induce surface image charges in the substrate, caused by the charge density of the plasmon. The magnitude of the substrate image charge will be screened by the factor $\left(\varepsilon_{s}-1\right) /\left(\varepsilon_{s}+1\right)$, where $\varepsilon_{s}$ is the permittivity of the substrate, a perfect image being obtained as $\varepsilon_{S} \rightarrow \infty .{ }^{46}$ This weak interaction between the plasmon and its image charge is sufficient to lift the degeneracy of the plasmon modes of the nanoparticle, producing new modes which are red-shifted or blue-shifted of the original position. ${ }^{18,47}$ While a number of theoretical approaches have been used to describe this system, ${ }^{48}$ the plasmon hybridisation model once again provides an intuitive way to understand the system. $^{32,47}$

As displayed in a previous section, plasmon hybridization describes the interaction of neighbouring plasmons by the creation of hybrid bonding and anti-bonding combinations of the original plasmon. ${ }^{32}$ In addition to the describing the interacting nanoparticle dimers can be applied to a variety of systems such as a nanoparticle and metal film, ${ }^{47}$ nanoparticle and metallic shell, ${ }^{33}$ and in the current case of a nanoparticle over a dielectric substrate. ${ }^{18,46}$

This approach consists of two steps; first as a nanoparticle approaches a surface the spherical symmetry of its plasmonic modes are broken and the degeneracy is lifted, producing 
$2 l+1$ modes, where $l$ is the order of the mode. The symmetry breaking induced by the substrate then facilitates the hybridisation between different order modes. When modes introduce a similar image charge distribution on the surface, the substrate mediates mixing of these modes, producing new hybrid modes. When a shaped nanoparticle, such as a cube, is used as opposed to a spherical particle, charges induced in the substrate occupy a larger area, resulting in a stronger interaction. Additionally a greater multitude of interactions may occur owing to the increased number of plasmon modes present in a cube. The most important modes in hybridization however are the dipolar mode and normally non-resonant quadrupolar mode, as these share a similar surface charge distribution and the dipolar mode is the most optically active. An additional advantage of the silver nanocube is that the dipolar and quadrupolar mode are nearly degenerate, they differ very little in energy and thus allow for a greater degree of hybridization in the new mixed modes. ${ }^{18}$

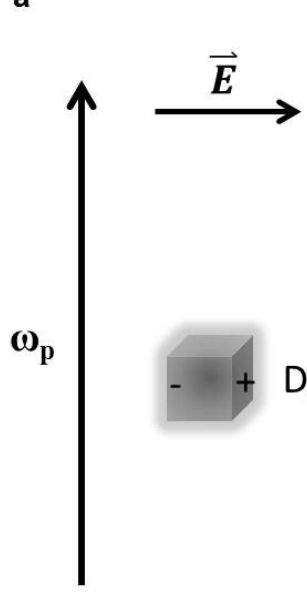

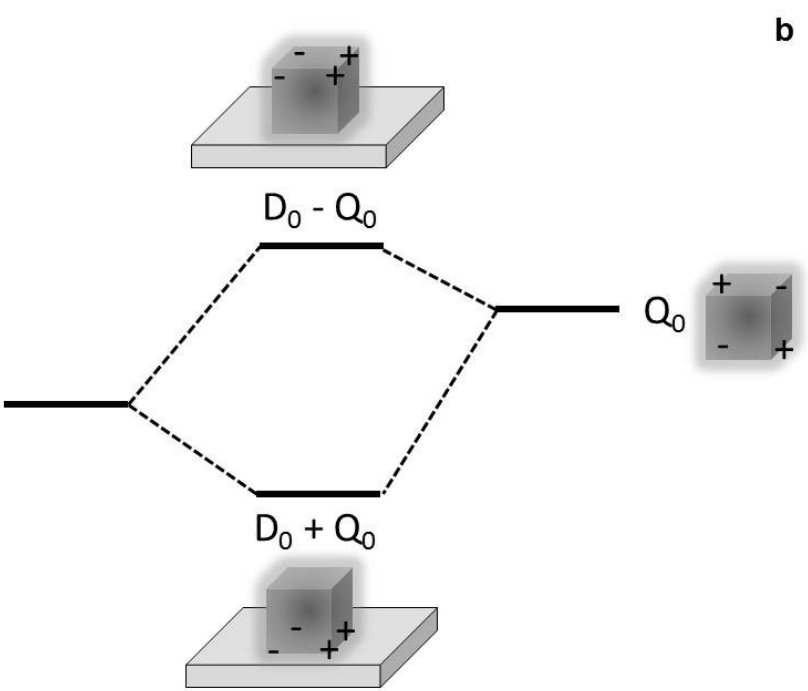

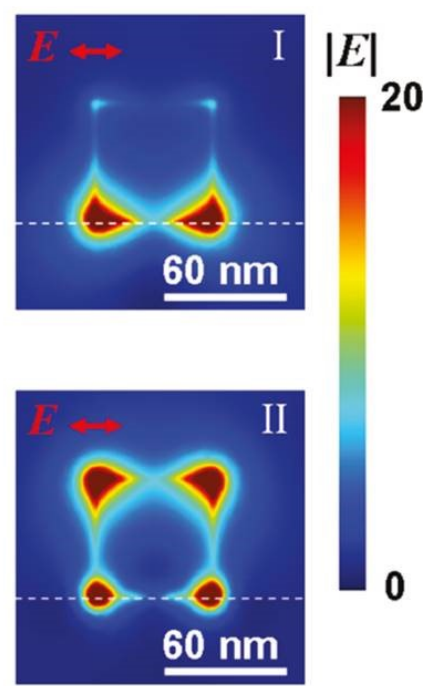

Figure 1.6 - a) Plasmon hybridization scheme of a nanocube interacting with a dielectric substrate. The substrate mediates interactions between the $\mathrm{D}_{0}$ and $\mathrm{Q}_{0}$ modes, producing a bonding $\left(\mathrm{D}_{0}+\mathrm{Q}_{0}\right)$ and antibonding $\left(\mathrm{D}_{0}-\mathrm{Q}_{0}\right)$ combination of mixed modes. The $\mathrm{D}_{0}+\mathrm{Q}_{0}(\mathrm{D})$ mode is primarily of dipolar nature and exists near the substrate, while the $\mathrm{D}_{0}-\mathrm{Q}_{0}(\mathrm{Q})$ mode is primarily quadrupolar in nature and exists away from the substrate. b) electric field intensity of the I) hybridized D mode and II) hybridized Q mode, displaying the spatial separation of the hybridized modes. Figure adapted with permission from $\left({ }^{18}\right)$, (C) ACS 2011 
Figure 1.6 displays the mixing of the original dipolar $\left(\mathrm{D}_{0}\right)$ and quadrupolar $\left(\mathrm{Q}_{0}\right)$ modes, resulting in bonding $\left(\mathrm{D}_{0}+\mathrm{Q}_{0}\right)$ and anti-bonding $\left(\mathrm{D}_{0}-\mathrm{Q}_{0}\right)$ modes. The bonding mode originates primarily from the $\mathrm{D}_{0}$ mode and is formally referred to as the hybridized dipolar (D) mode, while the anti-bonding mode is mainly $\mathrm{Q}_{0}$ and referred to as the hybridized quadrupolar $(\mathrm{Q})$ mode. As the interaction with the substrate increases, the hybrid D mode will show more $\mathrm{Q}_{0}$ character and vice versa for the hybridized Q mode. ${ }^{18}$ This is a useful consequence of the mode mixing, as since the $\mathrm{Q}_{0}$ mode is typically dark, the imparting of dipolar character to it through hybridization allows it to be excitable by incident radiation. ${ }^{46}$ An important consequence of this mode mixing is that these modes display different localizations of their charge density. The hybridized D mode has most its charges localised to the high refractive index environment, typically a substrate, while the Q mode exists distal from the substrate. ${ }^{18,49}$ This has important ramifications in sensing applications, as the $\mathrm{D}$ mode becomes sensitive to the refractive index of the substrate, while the Q mode becomes particularly sensitive to the surrounding environment. ${ }^{2,18,49}$ Further enhancement of the refractive index sensitivity of the Q mode can be made by increasing the refractive index of the substrate..$^{50,51}$ This increases the degree of hybridisation and spatial separation of the hybrid modes, allowing for increased refractive index sensitivity of the silver nanocubes, or other supported nanostructures. ${ }^{2,49-52}$ 


\subsection{Synthesis of Silver nanostructures}

Over the past decade, the shape controlled synthesis of silver nanocrystals has developed significantly. The field has advanced far past the synthesis of simple spherical particles, silver

nanocrystals can now be synthesized in virtually every conceivable geometric shape. ${ }^{10,53}$ A wide variety of methods to synthesize these particles accompany the wide variety of shapes and sizes that can be produced, but they all share a common approach. They all typically involve the use of a silver salt precursor, $\mathrm{AgNO}_{3}$ being the most common, a reducing agent, and a capping agent that may act as a stabilizer and/or shape-directing agent.

One of the oldest and most popular approaches to synthesizing silver nanoparticles is the citrate reduction method. ${ }^{54}$ In this method a set amount of sodium citrate is added to a boiling aqueous solution of $\mathrm{AgNO}_{3}$, where citrate acts as both the reducing agent and stabilizing agent. ${ }^{55}$ This method remains popular to this day due to the relative ease and simplicity of the reaction, but the produced colloids tend to be polydisperse in size or shape and thus not suited to extensive ensemble studies in plasmonics. ${ }^{55}$

A similar, simple approach is the reduction of $\mathrm{AgNO}_{3}$ by oleylamine (cis-1-amino-9octadecene). Like in the citrate reduction, oleylamine acts as both the reducing agent and stabilizer, and can even be used as the solvent, though organic solvents such as $n$-hexane, or liquid paraffin can be employed. ${ }^{56,57}$ The quick reduction of $\mathrm{Ag}$ at high temperature $\left(150^{\circ} \mathrm{C}-\right.$ $180^{\circ} \mathrm{C}$ ), in this synthesis yields highly monodisperse but small nanoparticles due to the high density of nuclei that are rapidly produced and grown, with Ostwald ripening serving to narrow the size distribution of the particles. ${ }^{57}$ 


\subsubsection{Polyol Method}

While the above methods of producing silver nanoparticles are capable of easily producing simple silver colloids, they are limited in their control of size and/or shape of the resultant nanocrystals. One of the most versatile methods in colloidal nanoparticle synthesis is the so-called polyol synthesis. In this method a preheated polyol, typically ethylene glycol (EG), is used both as the solvent and principle reducing agent in the action. ${ }^{10}$ By varying reaction conditions such as temperature, capping agent, reagent concentrations, and presence of trace ions it is possible to generate a large variety of shapes and sizes of silver nanostructures. ${ }^{10}$ As such it is the most popular method for generating silver nanostructures with controlled shape including nanospheres, bipyramids, polyhedrons, nanowires, and most importantly to this investigation, silver nanocubes. ${ }^{10,11}$

\subsubsection{Reduction Mechanism of Ag in the Polyol Method}

As with most silver colloid syntheses, this reaction begins with the reduction of $\mathrm{Ag}^{+}$from a silver precursor. While ethylene glycol alone can be used to reduce the Ag precursor at an elevated temperature, closer investigation suggested the primary reducing agent could be

glycoaldehyde produced by the in-situ oxidation of ethylene glycol. ${ }^{58}$ This compound is formed when ethylene glycol is heated in the presence of $\mathrm{O}_{2}$ naturally dissolved in the EG:

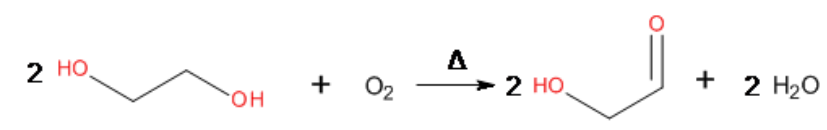

Ag can be subsequently reduced by the produced glycoaldehyde:

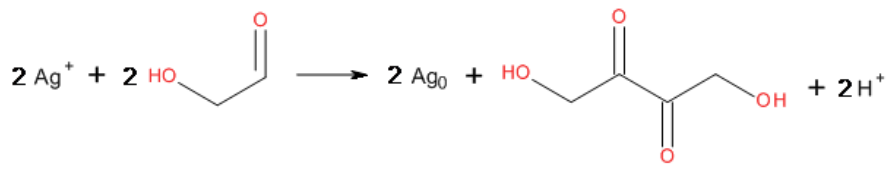


The production of glycolaldehyde is temperature and atmosphere sensitive, this mechanism thus explains why the same sensitivity is observed in the polyol synthesis of silver nanostructures. Note that this is not the only mechanism for reduction of $\mathrm{Ag}$ in this environment. Reduction of Ag still occurs in the absence of glycolaldehyde, albeit at reduced rates. The initial belief was that reduction of $\mathrm{Ag}$ occurred through the production of acetaldehyde from EG, though the presence of acetaldehyde has not been confirmed at the typical temperatures of the polyol synthesis. ${ }^{58}$ Nevertheless, no matter by what mechanism it may be, the polyol process has been demonstrated for the reduction of a number of metals, allowing for the formation of nuclei and subsequent growth of nanocrystals.

\subsubsection{Nucleation of Nanoparticles}

The nucleation of colloidal silver nanocrystals begin with the continuous reduction of a supply of $\mathrm{Ag}^{+}$ions to $\mathrm{Ag}^{0}$. The solution becomes quickly saturated with $\operatorname{Ag}^{0}\left(C_{s}\right)$ but cannot spontaneously produce solid nuclei until a critical concentration $\left(C_{n u}\right)$ due to the energy cost associated with this entropy change of the system. ${ }^{59}$ Once this concentration is met, stable nuclei are formed. Continued growth of the nuclei can now readily occur at a lower concentration of $\mathrm{Ag}^{0}$. Nucleation and growth continue simultaneously as two relatively separate processes, as long as the $\mathrm{Ag}^{0}$ concentration remains above $C_{s}$. This condition is usually met under normal reaction conditions as solubilized $\mathrm{Ag}^{0}$ is supplied by the continued reduction of the much more soluble, and thus plentiful, $\mathrm{Ag}^{+}$ions. If the $\mathrm{Ag}^{0}$ concentration drops below saturation, growth will continue only on existing nuclei. This model of describing the nucleation of nanocrystals is summarized in Figure 1.7. 


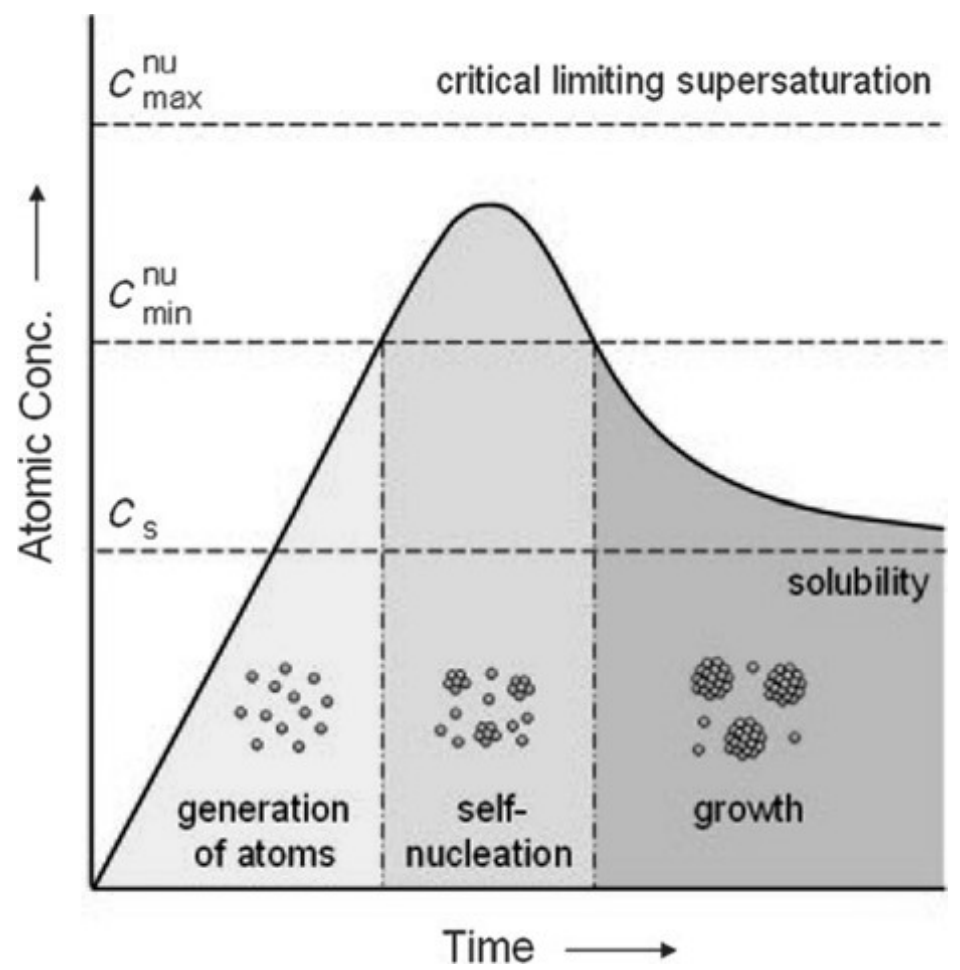

Figure 1.7 - Model describing the nucleation and growth of nanocrystals based on atomic concentration of the metal. Adapted with permission from $\left({ }^{59}\right)$, C ACS 1950, reproduced with permission from $\left({ }^{60}\right)$

The initial nuclei formed in the synthesis are small $(\sim 2 \mathrm{~nm})$ and are able to fluctuate between single crystal and twinned crystal morphologies. ${ }^{61}$ As the nuclei grow in size, the morphological fluctuations reduce until their structure becomes locked into the form either a single crystal, singly twinned, or multiply twinned crystal. Though they take longer to form, defects in twinned seeds enable a lower surface energy and will become the most abundant morphology in the system, especially the multiply twinned dodecahedron. ${ }^{62}$ As displayed in Figure 1.8 the type of nuclei initiating the growth ultimately determines the final morphology of the silver nanocrystal. There exist two paths in controlling the shape of a growing nanocrystal:

1) Selection of the desired seed morphology by control of the reaction kinetics or selective etching of undesired nuclei

2) Using structure-directing agents that alter the surface free energy of specific crystal facets 


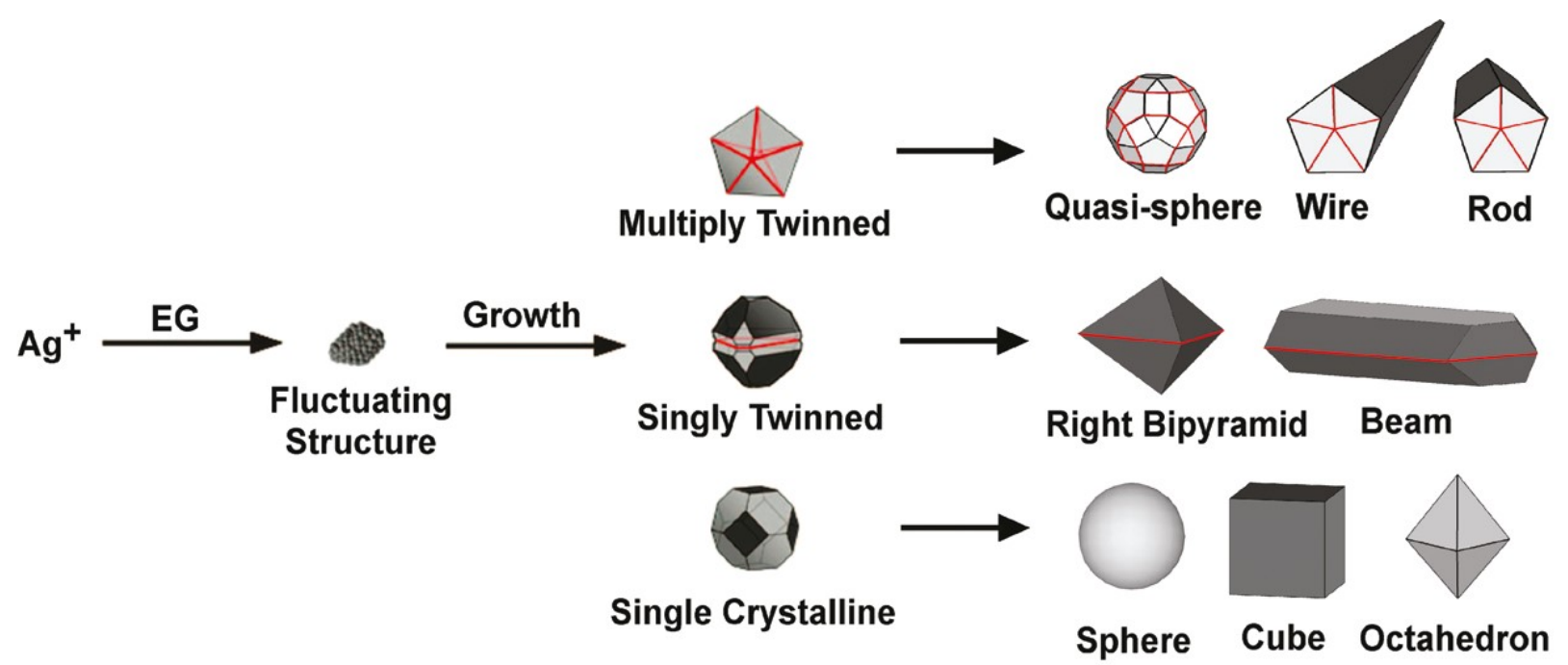

Figure 1.8 - Possible reaction paths in the polyol synthesis of Ag nanostructures. Initial nuclei formed after the reduction of $\mathrm{Ag}^{+}$may fluctuate between different morphologies until growth to a critical size at which their morphology is locked into a single crystalline, singly twinned, or multiply twinned seed. The resultant seeds may grow into many different morphologies depending on choice of structure directing agent and presence of certain trace ions. Reproduced with permission from $\left({ }^{10}\right)$ (C) ACS 2011

\subsubsection{Shape Controlled Growth and the Function of the Capping Agent}

Colloidal nanoparticles require some sort of surfactant or capping agent to prevent their spontaneous aggregation. A capping agent may also serve an additional function, to direct the shape of resultant nanoparticle. This is done by selective binding to a specific crystal facet, passivating the surface and lowering the growth rate along that crystal plane. For example it has been shown that PVP and $\mathrm{Br}^{-}$ions will selectively bind to the $\{100\}$ facet of $\mathrm{Ag}$ and other fcc metals. ${ }^{63,64}$ Conversely, citrate has been shown to bind selectively to the $\{111\}$ facet of Ag. ${ }^{63,65}$ The surface energies of crystal facets in fcc metals follow the order $\gamma\{111\}<\gamma\{100\}<\gamma\{110\}$, and growth of these crystals will typically be dominated by a mixture of $\{111\}$ and $\{100\}$ facets. When a facet is passivated by a surface species, for example the $\{100\}$ facet, incoming metal atoms will preferentially bind to the non-passivated $\{111\}$ facet during growth. These adatoms will then migrate to the face edges, resulting in elongation of the $\{100\}$ facets, which in single crystal seeds leads to the formation of nanocubes. ${ }^{66}$ Conversely passivation of the $\{111\}$ facet, 
such as when using citrate, will produce octahedrons for single crystal seeds. ${ }^{63}$ Further morphologies can be produced if anisotropic growth can be induced, such as nanobars, ${ }^{67}$ and nanowires. ${ }^{68}$ While not all the mechanisms for anisotropic growth are well understood, ${ }^{11}$ multiply twinned seeds have been shown to preferentially grow into nanorods and nanowires with pentagonal cross section when using a $\{100\}$ facet pacifier, such as $\mathrm{Br}^{-}$or $\mathrm{PVP}^{69}$ Due to the high strain on surface atoms located away from the center of the dodecahedral face, growth will preferentially occur parallel to the center axis providing there is passivation of the $\{100\}$ facet. $^{69}$

\subsubsection{Seed selection through Oxidative Etching}

One possible method to control the shape of nanoparticles during growth is to limit which morphology of seed is available for growth. In 2004, Xia et al. demonstrated that the combination of $\mathrm{Cl}^{-}$and $\mathrm{O}_{2}$ in solution can be used to selectively etch twinned seeds. ${ }^{70}$ This combination has been shown to be able to dissolve silver nanocrystal nuclei. It is believed the chloride acts as a coordinating ligand which facilitates oxidation of the silver by dissolved oxygen. ${ }^{70}$ The dissolvation of silver nuclei is selective towards singly twinned and multiply twinned seeds as these geometries often contain defects which serve as energetic sites for the chloride coordination. It has been well demonstrated that the use of trace amounts of $\mathrm{NaCl}$ or $\mathrm{HCl}$ produce singly crystal particles, resulting in a higher population of crystals with cubic morphology when PVP is used as a capping agent. ${ }^{70}$ By substituting $\mathrm{NaCl}$ with $\mathrm{NaBr}$, singly twinned seeds are selected, resulting in a right bipyramid morphpolgy. ${ }^{71}$ The use of Br ${ }^{-}$allows for the selective etching of multiply twinned seeds but not singly twinned seeds, and has been shown to act as a weaker ligand in the oxidative etching system than $\mathrm{Cl}^{-}$. 


\subsubsection{Kinetically Controlled Selection of Seeds}

It has been demonstrated that trace amounts of of $\mathrm{S}^{2-}$ in the polyol synthesis significantly increase the reaction rate. ${ }^{72}$ This is caused by two effects: first, due to the strong interactions of sulfide species with silver, $\mathrm{Ag}_{2} \mathrm{~S}$ nuclei rapidly form, quickly allowing sites for further growth of the silver nanocrystals. Secondly, $\mathrm{Ag}_{2} \mathrm{~S}$ nanoparticles have been shown to catalyze the reduction of $\mathrm{Ag}^{+}$by drastically reducing the reduction potential of $\mathrm{Ag}$ compared to that of free $\mathrm{Ag}^{+}{ }^{73}$ When a trace amount of $\mathrm{Na}_{2} \mathrm{~S}$ or NaHS is added to the polyol synthesis in the presence of PVP, a high yield of single crystal silver nanocubes are obtained, even without the presence of an oxidative etchant. This is the result of kinetic control over the selection of seeds. Due to the increased strain on these structures caused by the twin defect, they cannot grow as rapidly as single seeds. Thus rapid growth will be selective towards the production of nanocrystals with single seed morphology. Likewise, drastically reducing the reaction rate, such as through the use of a weak reducing agent, will make the reaction selective towards multiply twinned seeds, producing shapes that are not typically favoured by thermodynamics, such as plates. $^{74}$

\subsubsection{Seed Mediated Synthesis}

A problem that occurs particularly in the growth of larger nanocrystals, is difficulty in controlling the monodispersity of both size and shape of the final particles. A possible means to remedy this effect is through the seed-mediated synthesis of the nanocrystals. ${ }^{75}$ In this sort of synthesis, pre-synthesized nanoparticles are injected into the reaction just prior to the addition of additional silver precursor. Further growth of these nanoparticles occurs without the necessity of the lengthy nucleation step. This method can be used to grow new samples with a larger size, ${ }^{75}$ different shape, ${ }^{76}$ or improve monodispersity through Ostwald ripening. ${ }^{77}$ 


\subsection{Assembly of Nanocrystals}

While the properties of individual nanocrystals are interesting on their own, control over their assembly on a solid substrate is useful for both the study of their properties and for their design in potential applications. One of the most important properties to control, and the most pertinent to this work, is control of the interparticle distance. The plasmonic properties of coupled nanostructures are of particular interest in the application of surface-enhanced Raman spectroscopy, where the many-fold increase in electric field intensity in the junction can lead to enormous enhancement of Raman scattering. Thus the ability to engineer gaps in nanocrystal assemblies is of the utmost importance. While there are numerous strategies for the assembly of plasmonic nanomaterials, this work will focus primarily on self-assembly, and the technique used most in this work, the Langmuir-Blodgett technique.

\subsubsection{Self-Assembly}

The most common way of organizing plasmonic nanocrystals is through self-assembly due to its simplicity and versatility. Self-assembly relies on the utilisation and careful control of the forces between nanoparticles and their environment. With sufficient control and design of the nanoparticle surfaces and environments, assemblies including dimers, ${ }^{78}$ linear chains, ${ }^{79}$ 2D lattices $^{80}$ and 3D superstructures ${ }^{81}$ can be formed spontaneously.

Self-assembly is the spontaneous organisation of nanocrystals driven by the minimization of free energy in the system (i.e. minimizing $\Delta G=\Delta H-T \Delta S$ ). The enthalpy $(\Delta H)$ component of the system represents the interactions between nanoparticles while the entropy $(\Delta S)$ represents the free volume of each nanoparticle. The inter-nanoparticle interactions, typically governed by van der Waals forces, tend to be attractive, while entropy may contribute to either attractive or repulsive forces. The predominant entropic contribution is in the form of steric repulsion 
contributed from the capping agents of colloidal nanoparticles. Entropy can contribute to attractive forces as well however, for example when the average inter-particle distance is smaller than the dimension of the particles In this instance a transition from the disordered phase to the ordered phase occurs in order to gain more free volume for each particle, tending to form closepacked arrays of nanoparticles dependent on their shape. ${ }^{82}$

The van der Waals forces between metal nanoparticles are the most significant interaction that occurs between them, especially magnified by the ease of formation of induced dipoles. If left unchecked this interaction would form disordered aggregation of the nanoparticles, hence why colloidal nanoparticles utilize stabilizing surface molecules to protect from this. ${ }^{82}$ In addition to providing the steric repulsion necessary to keep colloidal nanoparticles stable, these surface molecules also induce surface charges which result in electrostatic interactions are dependent on $\mathrm{pH}$, ionic strength and electrolyte presence. ${ }^{83,84}$ Control over the surface charge of nanocrystals provides a possible route to controlled self-assembly on nanocrystal ensembles. The capability of post-synthetic modification of the surface molecules on a nanoparticle with alternate molecules, macromolecules/polymers, and biomolecules also allow the utilization of a variety of different interactions to control nanoparticle assembly, such as hydrogen bonding, covalent bonds, Watson-Crick base pairs, and other bio-specific interactions. ${ }^{82}$ Another important means of self-assembly is through the use of solvency forces, specifically poor solvency or the "hydrophobic" or "solvophobic" forces. ${ }^{85}$ Introducing nanoparticles to an environment where their surface molecules are poorly soluble will maximize their entropy by forcing the aggregation of nanoparticles.

This last class of interactions represents one of the most well established routes of selfassembly of noble metal nanoparticles. ${ }^{86}$ Utilisation of self-assembled monolayers (SAMs) of 
alkanethiols on the surface of gold or silver nanoparticles provides a simple route for the assembly of continuous 2D lattices of nanoparticles. ${ }^{80,87}$ Thiols are the functional group of choice for replacing surface molecules on noble metal molecules, as the covalent nature of the $\mathrm{Ag}-\mathrm{S}$ or $\mathrm{Au}-\mathrm{S}$ bond is significantly more energetic than the weak physical or electrostatic forces that keep the native stabilizers bound to the nanoparticle surface. The Ag and Au possess the capacity to form to form well-ordered SAMs of alkanethiols, ${ }^{86}$ leading to exceptionally strong interactions between nanoparticles, especially those with large, flat facets. ${ }^{88}$ This sort of system provides excellent control, as by altering the chain length of the alkanethiol it is possible to tune interparticle distances. ${ }^{87}$ Furthermore, multicomponent functionalization allows routes to unique assemblies, such as the demonstration of selectively functionalizing the face of $\mathrm{Ag}$ nanocubes with hydrophobic and hydrophilic SAMs, allowing for formation of dimers, tetramers and other unique configurations. ${ }^{89}$

\subsubsection{Assembly at an interface}

While self-assembly is often performed in solution or by solvent evaporation, assembly at an interface such as the liquid/air or liquid/liquid interface can allow for greater control of the assembly process. Such an interface acts as a "soft substrate" giving the particles sufficient mobility to arrange themselves before being transferred to a solid substrate. Rearrangement of the nanoparticles at the interface can either be done via self-assembly, such as with alkanethiol capped nanoparticles, ${ }^{80,90}$ by increasing the surface concentration of nanoparticles to invoke a disordered to ordered phase transition, ${ }^{91}$ or by applying an external shear force, as in the Langmuir-Blodgett technique. ${ }^{92}$ 


\subsubsection{The Langmuir-Blodgett Technique}

Originally intended for the study of amphipathic molecules, the Langmuir-Blodgett (LB) technique involves the dispersion of a monolayer at the air/water interface followed by its manipulation by a movable barrier and deposition on a solid support (Figure 1.10). This technique which has now been widely applied to use with hard nanomaterials, ${ }^{92}$ offers excellent control to the packing of a monolayer through the application of lateral surface pressure. The Langmuir-Blodgett technique is the preferred assembly approach used in this work due to the exceptional control it provides over the optical properties of plasmonic nanomaterials. ${ }^{93}$ Noble metal nanoparticles are well-suited to the technique as they frequently employ organic stabilizing molecules which are often sufficiently hydrophobic for dispersal at the air/water interface. Silver nanocubes are particularly good example of this, as their native stabilizer, polyvinylpyrrolidone (PVP), has a good affinity for water, because of the polar pyrrolidone moiety, but the long carbon chain is sufficiently hydrophobic for use with LB, producing a well-dispersed monolayer which experiences little to no spontaneous aggregation upon solvent evaporation. ${ }^{94}$ However some aggregation does occur upon compression of these monolayers as even the weak hydrophobic forces are sufficient to induce this between the large facets of the nanocubes.

Langmuir-Blodgett monolayers are controlled by an isothermal compression, where the linear surface pressure is monitored by a Wilhelmy plate. The LB isotherm, an example of which is displayed in Figure 1.9, is often described in terms of analogue phases. The gas-phase is characterised by a zero or near-zero pressure and slope in the isotherm, in which the particles have large free volumes and do not interact much with each other. As the pressure increases, the liquid phase is approached and the isotherm develops a gradual slope as interparticle interactions increase, though the particles are still relatively mobile. There is sometimes a phase transition 
between the liquid-expanded and liquid-condensed phase, related to reorganisation of the particles as their average free volumes approach their dimensions, observed as a plateau in the slope in the isotherm. At high surface pressures the mobility and free volumes of the particles become extremely limited, and they enter the solid phase which is characterized by a near vertical slope in the isotherm. The example presented in Figure 1.9 represents the LB isotherm of PVP capped AgNC, however the monolayer's behaviour, isotherm, and final morphology is a consequence of the interparticle forces in the system, and can be modified by changing the particle's size, geometry, capping agent, and other materials in or on the subphase.

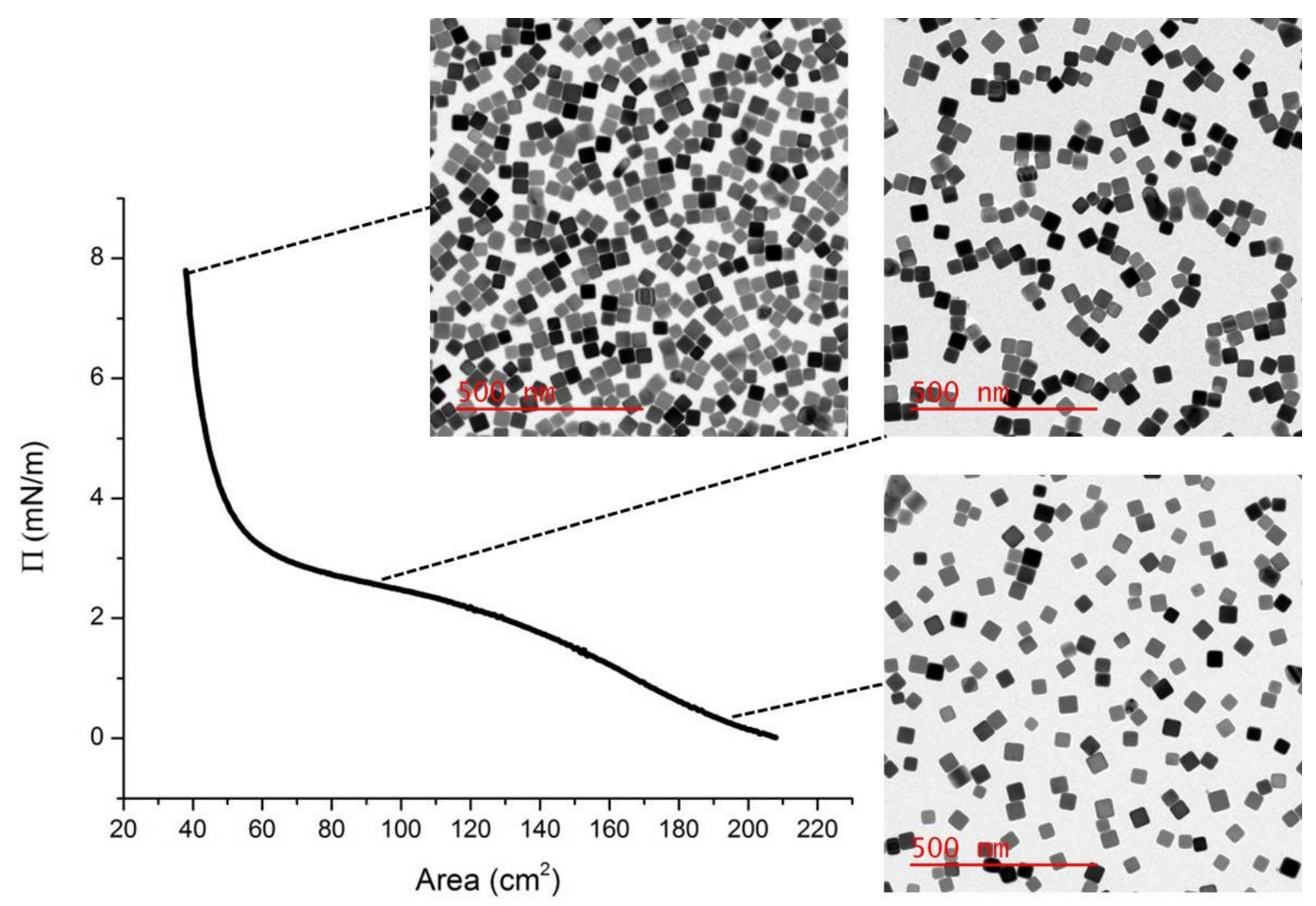

Figure 1.9 - Langmuir-Blodgett isotherm (linear surface pressure, $\Pi$ v.s. physical area of the trough) of a silver nanocube monolayer. As the trough barrier is compressed the surface pressure and packing density of the monolayer increases. The monolayer's state may be described by analogue-phases which can be indicated by the slope of the isotherm. TEM images of monolayers deposited at the solid, liquid, and gaseous analogue phases are displayed. 
Deposition of the resultant monolayer onto a solid substrate is accomplished by vertical dipping. Typical deposition is done by vertical lifting of the substrate after it has been submerged, with the material being deposited as the substrate is slowly lifted out of the meniscus. This type of deposition only properly applies to hydrophilic substrates due to the requirement of a positive meniscus. Alternatively the Langmuir-Schaefer technique can be employed, in which the Langmuir film is horizontally lifted off by the substrate. This method is more suitable when the monolayer and substrate are hydrophobic.

The control of Langmuir-Blodgett monolayers is not limited to just the isothermal compression of the Langmuir film. The resultant nanoparticle monolayers can be patterned by post-deposition lithography ${ }^{95}$ or control of the assembly through the addition of hydrophobic or amphipathic molecules to the monolayer. ${ }^{96,97}$ Our monolayers make use of a passive phospholipid spacer which has been shown to reduce aggregation of silver nanocubes due to its affinity to PVP and natural existence in the liquid-expanded phase. ${ }^{51}$
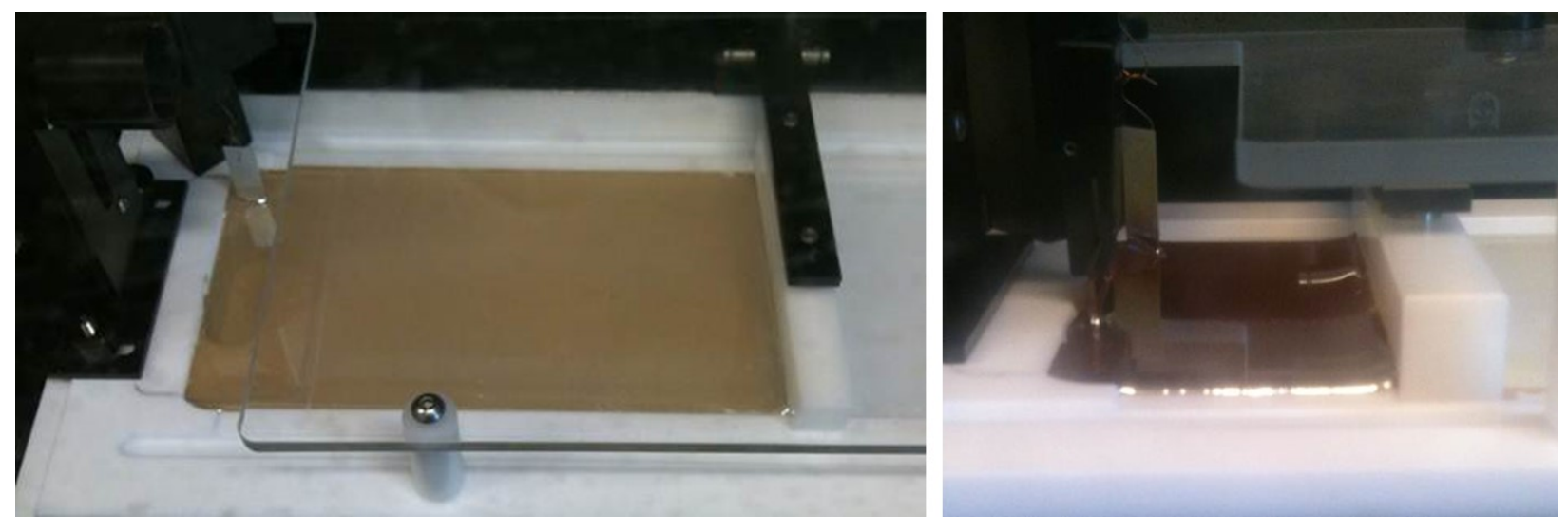

Figure 1.10 - Photographs of a silver-nanocube monolayer on dispersed at the air/water interface on a LangmuirBlodgett trough. The left monolayer is a well-dispersed monolayer in the liquid phase. As the monolayer is compressed the packing density is increased, resulting in an opaque metallic-looking monolayer. 


\subsection{Surface Enhanced Raman Spectroscopy (SERS)}

Surface-enhanced Raman spectroscopy (SERS) is a technique that falls under a family of field-enhanced phenomena, including surface-enhanced second harmonic generation, ${ }^{98}$ infrared absorption, ${ }^{99}$ and fluourescence. ${ }^{100}$ All of these techniques are reliant on the near-field optical enhancement near the surface of plasmonic nanostructures.

Though not recognized at the time, the first observation of SERS was reported by Fleischmann, Hendra and Mcquillan in 1974 when using Raman spectroscopy to study the surface of a roughened silver electrode. ${ }^{101}$ The intense Raman signal of pyridine adsorbed to the surface of the electrode was initially attributed to the increased surface area by the electrode roughening procedure. ${ }^{102}$ In similar experiments Van Duyne and Jeanmaire reported Raman signals $10^{5}-10^{6}$ times higher than expected for monolayer of absorbed pyridine due to some unknown property of the electrode surface. ${ }^{103}$ Similar independent observations in by other groups of the period led to the understanding that the surface was enhancing the Raman scattering itself, this effect later being coined surface-enhanced Raman scattering (SERS). ${ }^{102}$ While promising, SERS did not develop initially to its full potential due to practical obstacles and gaps in theory and understanding. A limited few materials were shown to exhibit strong SERS, namely $\mathrm{Ag}, \mathrm{Au}$, and $\mathrm{Cu}$, which required surface roughness on the order of nanometers to a couple hundred nanometers to be effective, which was difficult to fabricate. Raman spectroscopy instrumentation was also not nearly as sophisticated and efficient as modern Raman instrumentation. ${ }^{102}$ It was not until the observation of single molecule SERS in 1997 independently by Nie et al. and Kneipp et al. that interest in the technique was reinvigorated. ${ }^{104,105}$ By this point the origins of the surface enhancement were becoming well understood, the primary mechanism being due to enhanced local electric fields at the surface 
caused by the presence of a LSPR. ${ }^{106}$ Now with the understanding of the origin of the SERS phenomenon and the demonstration of its potential, research in SERS has become a popular, multidisciplinary field of research, and has proven to be a versatile analytical technique with numerous applications and unexplored potential.

\subsubsection{The SERS Enhancement Factor}

One of the difficulties in the early days of SERS research was establishing the precise magnitude of the effect. The initial observations of single molecule SERS claimed enhancement on the order of $10^{14}$, and for some time it was assumed that enhancements on this order were necessary for single-molecule observations. ${ }^{105}$ More recent, rigorous treatment of the SERS enhancement factor have revealed that EFs on the order of $10^{7}-10^{8}$ are sufficient for single molecule SERS. ${ }^{107}$ Even to this day there is wide variability in EFs in the literature that arises from the way it is defined and estimated in practice. In this section we will fundamentally define the SERS EF and reveal where the variability in its interpretation arises.

The formal definition of the SERS enhancement factor is the factor by which the intensity of the Raman scattering from an individual molecule is enhanced at the surface against in solution:

$$
S E R S E F=\frac{I_{S E R S}}{I_{\text {Raman }}}
$$

where $I_{S E R S}$ and $I_{\text {Raman }}$ is the average Raman intensity per molecule in each respective case. To fundamentally examine where this enhancement arises from, we turn our attention to what determine the intensity of Raman scattering from a molecule. The specific intensity of a Raman signal can be described by the following equation: 


$$
I_{s}=I_{0} \frac{d \sigma_{j}}{d \Omega} D(d z) A_{D} \Omega K
$$

where $I_{0}$ is the intensity of the excitation source, typically expressed as power density (photons $\left.\mathrm{cm}^{-2} \mathrm{~s}^{-1}\right), D(d z)$ is the number density of scatters, i.e. concentration or surface concentration of molecules, $d \sigma_{j} / d \Omega$ is the differential Raman cross section (sometimes denoted as $\beta$, expressed in $\mathrm{cm}^{2}$ molecule $\mathrm{sr}^{-1}$ ) of the $j$ th vibrational mode. $A_{D}$ and $\Omega$ are the area of collection $\left(\mathrm{cm}^{2}\right)$ and the solid angle of collection ( $\mathrm{sr}$ ) respectively. $K$ is a constant specific to instrumental parameters such as depth of field, transmission though the optics, detection efficiency and so forth. The number density, collection volume, and other instrumental parameters cannot contribute to enhancement themselves, while $I_{0}$ refers to the intensity of the incident light in the far-field. Therefore the observation of SERS enhancement is the apparent increase in Raman crosssection:

$$
S E R S E F={\frac{d \sigma_{j}}{d \Omega_{S E R S}}} /{\frac{d \sigma_{j}}{d \Omega_{\text {Raman }}}}
$$

Thus by comparing the SERS cross section of the molecule with the Raman cross section one can determine the enhancement factor. This can be done by comparing Raman intensity against a standard with a known enhancement factor, ${ }^{108}$ or by more complex means such as vibrational pumping. ${ }^{109}$ For the majority of SERS applications and experiments however, one is dealing with average SERS signals in an ensemble measurement. The rigorous calculation of the SERS cross section may not be practical or accurate. The most widely used definition of the SERS EF is: ${ }^{110}$

$$
\text { SERS EF }=\frac{I_{\text {SERS }} / N_{\text {SERS }}}{I_{\text {Raman }} / N_{\text {Raman }}}
$$


Essentially we have returned to the basic definition of SERS enhancement, the ratio of average Raman intensities per molecule, though what differs is that in practice the interpretation of equation (12) is subjective to the experimenter. How the molecules have been deposited on the SERS substrate, how the experimenter considers the substrate and what is used as the Raman reference will ultimately define $N_{S E R S}$ and $N_{\text {Raman }}$. This represents one of the fundamental problems with this representation of the enhancement factor. For example in the determination of $N_{S E R S}$ does one consider only molecules adsorbed to the metals or to the entire 2D planar surface? Or in the case of $N_{\text {Raman }}$, which is typically taken from a liquid sample, how is the scattering volume estimated? If the treatment of these terms vary significantly between experiments, artificial variations in the EF may be separated by as much as 2 orders of magnitude. ${ }^{110}$

Even in this work there exist variations into how we define and treat the SERS EF in consideration of practical experimental conditions. A rigorous definition of how SERS EFs are calculated in this work is available in Chapter 3 as they become relevant, however it is important to note that the SERS EF in principle is semi-qualitative, and is used to compare trends in observed results as opposed to an absolute quantity which can invariantly define the quality of a SERS substrate. 


\subsubsection{Electromagnetic (EM) Mechanism of SERS enhancement}

SERS differs from normal Raman spectroscopy primarily in its requirement for the presence of a metal nanostructure. While SERS has been demonstrated on a number of metal surfaces, such as $\mathrm{Ag}, \mathrm{Au}, \mathrm{Cu}, \mathrm{Li}, \mathrm{Na}, \mathrm{K}, \mathrm{In}, \mathrm{Pt}$, and more, it has always been found that the best enhancement occurs when using $\mathrm{Ag}$ or $\mathrm{Au}$ as the substrate material. ${ }^{102,106}$ Considering that most Raman instrumentation uses visible light excitation, and that these two metals are known for the strong plasmons in the visible spectrum, it is no surprise that the presence of an LSPR is the main prerequisite for strong SERS enhancement. Indeed the primary source of enhancement in SERS is due to the enhanced local electric fields at the surface of a plasmonic nanostructure. Maximizing this EM enhancement is the goal when designing and fabricating SERS substrates, and in many cases the SERS enhancement factor itself is essentially a measure of the average Efield enhancement of the system. ${ }^{3,106,111}$

The electric field enhancement originates from the interaction of incident radiation with a surface plasmon resonance. The magnitude of the induced dipole of the plasmon, $\mu_{\text {metal }}$, is determined by the polarizability of the metal, $\alpha_{\text {metal }}$, and the strength of the incident electric field, $E_{0}: \quad \mu_{\text {metal }}=\alpha_{\text {metal }} E_{0}$. This dipole of course resonates with the angular frequency of the incident light, $\omega_{0}$, and is capable of re-emitting radiation at the same frequency. This elastic scattering caused by the coherent interaction with the incident electric field, $E_{0}$, results in a spatial distribution in field charges in which light intensity is depleted from space surrounding the nanoparticle (measured as far-field extinction) but enhanced in localized areas near it (i.e. near-field). ${ }^{34}$ This produces on an average enhanced field, $E_{\text {loc }}$, localized around the surface of the particle, which is enhanced by some factor relative to the original incident field, $E_{0}$. 
Now we look at the origins of Raman scattering to demonstrate how enhancement ensues this interaction. In a very similar manner as described above, the local electric field may induce a dipole in a molecule, its magnitude being described in the same manner by: $\mu_{\text {molecule }}=$ $\alpha_{\text {molecule }} E_{\text {loc }}$. Likewise a molecule may also respond to incident light of frequency, $\omega_{0}$, by elastic scattering, or Rayleigh scattering, of radiation at the same frequency. While this applies to the molecule as whole, a molecule will contain a total of $j=3 \mathrm{~N}-6$ normal vibrational modes for non-linear molecules or $j=3 \mathrm{~N}-5$ modes for linear molecules. Interaction with one of these

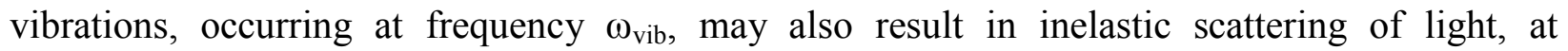
frequencies $\left(\omega_{0}-\omega_{\mathrm{vib}}\right)$ or $\left(\omega_{0}+\omega_{\mathrm{vib}}\right)$ which correspond to Stokes and anti-Stakes Raman scattering, respectively. Considering the time dependent electric field strength, $E=$ $E_{0} \cos 2 \pi \omega_{0} t$, and vibrational normal modes, $Q_{j}=Q_{0, j} \cos 2 \pi \omega_{v i b, j} t$, the polarizabilty description can be expanded to: ${ }^{112}$

$$
\mu=\alpha E_{l o c} \cos 2 \pi \omega_{0} t+E_{l o c} Q_{0, j}\left(\frac{\delta \alpha}{\delta Q_{j}}\right) \frac{\cos 2 \pi\left(\omega_{0}+\omega_{v i b, j}\right) t+\cos 2 \pi\left(\omega_{0}-\omega_{v i b, j}\right)}{2}
$$

From this equation we can note: Raman-active vibrations will only occur in oscillations where there is a change in polarizability $(\delta \alpha / \delta Q \neq 0)$. The intensity of the scattered light will be proportional to the polarizability of the vibrational mode, though note $\frac{\delta \alpha}{\delta Q_{j}} \alpha$ is still generally much smaller than $\alpha$, and Raman scattering is much weaker than Rayleigh Scattering. Most pertinent to this discussion however, is that the scattered light is proportional to the local electric field, $E_{l o c}$, near the molecule. Since a molecule is arbitrarily small in comparison to a metal nanoparticle, it benefits from the ability to experience the enhanced local electric field near the 
nanoparticle surface, leading to the many orders of magnitude increase in Raman scattering cross section.

The Raman scattered light may likewise be further enhanced by the nanoparticle by being elastically scattered into the far-field. The SERS intensity is thus dependent on the local field enhancement of both the incoming $\left(\omega_{0}\right)$ and outgoing $\left(\omega_{0}-\omega_{v i b}\right)$ electric field: $I_{S E R S}=$

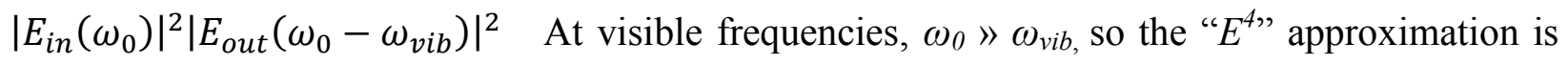
widely used to describe SERS intensity, where SERSEF $\approx\left|E\left(\omega_{0}\right)\right|^{4}$ is described by the enhancement of the electric field at that frequency. In practice, $\omega_{0}$ and $\omega_{0}-\omega_{v i b}$ are different enough to merit consideration, especially in the red and near-IR where they may be separated by tens of nanometers, or where plasmon resonances are particularly sharp.

\subsubsection{The SERS Hot Spot}

Even in early observations of SERS it was readily apparent that SERS-active surfaces displayed non-uniform enhancement. In fact it has been noted that only a minority of molecules absorbed to metal surfaces participate in the bulk of the enhanced signal. ${ }^{113,114}$ On a SERS active substrate, some localized spots exhibit significantly higher SERS activity than others. Moskovits, Shalaev and coworkers theoretically examined and directly observed these highly active areas by near-field optical microscopy and coined them as "hot spots". 115,116,117 These hot spots tend to occur in the gap between adjacent nanostructures. This is why SERS is seldom reported for individual nanoparticles, but often for aggregates, clusters, well defined arrays and dimers of nanoparticles.

The mutual interaction of the plasmon dipoles of adjacent nanostructures leads to the an increased magnitude in the induced dipoles and in the electric field intensity between them. 
While the typical $E^{4}$ electric field enhancement at the surface of a nanoparticle is on the order of the $10^{3}$, theoretical calculations predict enhancements as high as $10^{11}$ in the junction between adjacent nanoparticles with a $1 \mathrm{~nm}$ separation. ${ }^{118}$

The enhancement of $10^{11}$ is not a typical enhancement in nanoparticle junction however, but refers to the point of maximum enhancement. Recall that SERS hot spots are very localized, the ability to observe an enhancement of this magnitude is dependent on the probability of a molecule being at the hotspot. Considering a substrate with random placement of the target molecules, the probability of having any given SERS enhancement factor follows a long tail probability distribution as given in Figure $1.11 .^{107}$ Thus it is far more likely to record a signal from a molecule at regions of moderate enhancement than those at the hotspots. However due to the nature of the massive enhancement at the hot spot, signals from molecules near it will be account for a disproportionately large fraction of the collected SERS signal. Experimental investigations of this parity was done by Dlott and coworkers by measuring SERS of benzenethiol on silver-coated silica nanospheres sequentially after numerous high-intensity laser pulses were used photobleach the most enhanced molecules. ${ }^{119}$ They demonstrated that $90 \%$ of the BT molecules only contributed to $15 \%$ of the collected signal, while less than $1 \%$ of the molecules generated about $70 \%$ of the recorded Raman scattering. Or even more impressive is the fact that almost a quarter, 24\%, of the signal was generated by just 63 out of a million of the BT molecules experiencing a SERS enhancement of $>10^{9}$. Note, however, that the average enhancement factor of the ensemble prior to photobleaching was on the order of $10^{5}-10^{6}$. 


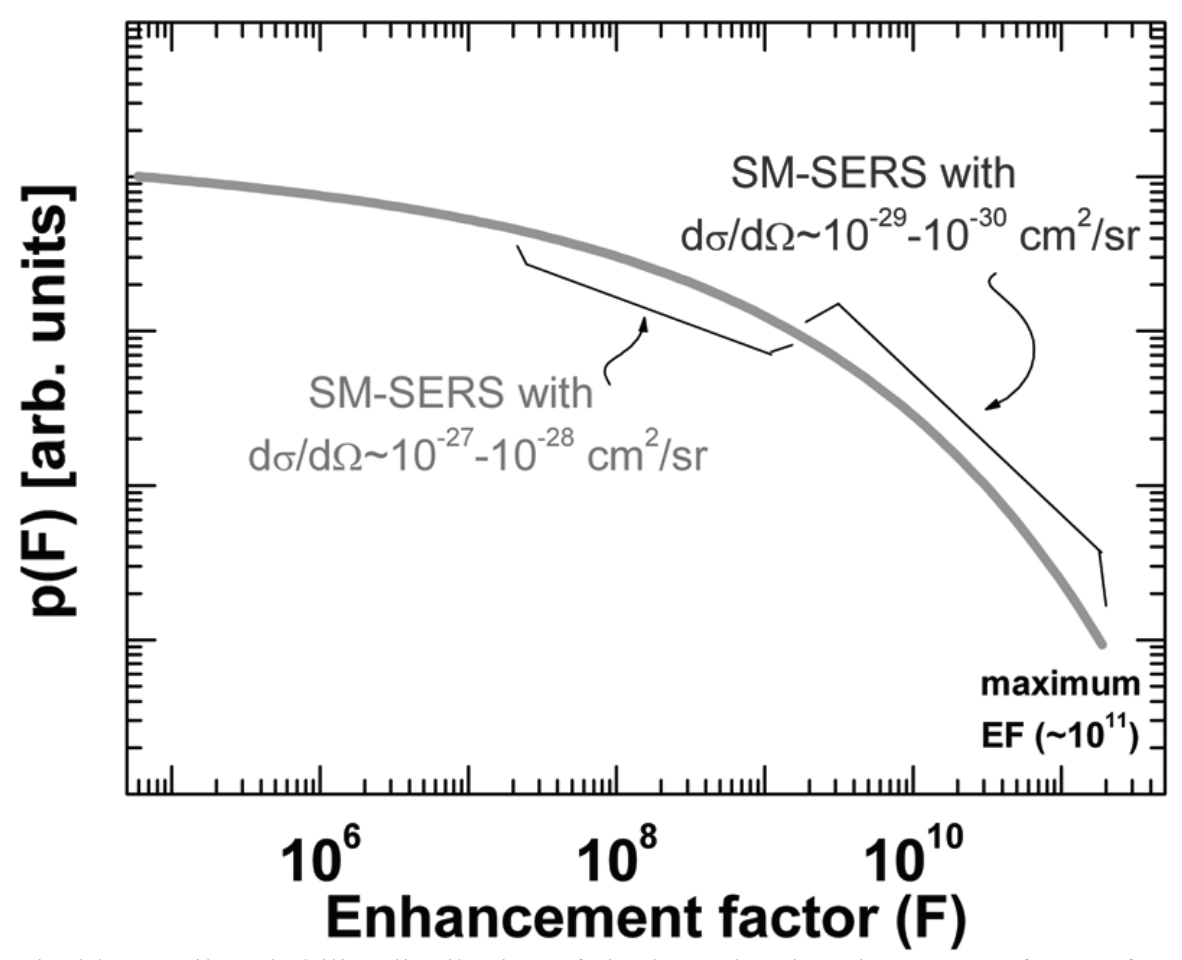

Figure 1.11 - Typical long-tail probability distribution of single-molecule enhancement factors for a SERs substrate capable of yielding enhancements up to $10^{11}$. The single-molecule regime is indicated with respect to the differential cross-section of the Raman vibration. Reproduced with permission from $\left({ }^{107}\right)$, (C) ACS 2009

This displays the importance of getting target molecules into the hotspots of the SERS substrates but what also must be considered is the number of molecules being measured. The above example demonstrates how using a large sampling volume of molecules makes it extremely likely to measure SERS from the hot spot, but significantly lowers the average enhancement factor of the ensemble. Conversely, most reported examples of single molecule SERS estimate 1 or less molecules in the scattering volume. ${ }^{120}$ While the reported enhancement factors in these cases are impressive, they are also impractical as recording such an event is extremely unlikely. ${ }^{121}$ In fact the average SERS events recorded (or too weak to be recorded) will dominate ensemble measurements, leading to a very low probability of recording a strongly enhancing event, and very large statistical variation in the results. As the number of molecules in the scattering volume increases the probability of observing an extreme enhancing event diminishes, but the statistical distribution of the SERS intensity improves. ${ }^{121}$ Thus by controlling 
the number of molecules on the substrate the experimenter can decide between a set of consistent, but average SERS enhancement factors, or combing through statistically invariant results for the maximal SERS enhancement or single-molecule detection events.

\subsubsection{Non EM-Enhancement: Chemical Enhancement}

The large SERS enhancement factors, up to $10^{14}$, initially reported for single molecule SERS, though impressive, cannot be explained by the electromagnetic enhancement mechanism alone. Theory predicts this sort of enhancement can only lead to a maximum of $10^{11}$, at very localized points in the junction between nanostructures. ${ }^{118}$ Thus at the time it was believed "chemical enhancement" effects are required to bridge the gap between the highest enhancement factors and those predicted by theory. A number of theories have developed in regards to the sources of this additional enhancement, typically involving charge transfer processes or new resonances induced by the chemisorption of the analyte to a metal. ${ }^{34,122}$ Additional evidence for the presence of these effects exist in the form of large differences in enhancement between similar physisorbed and chemisorbed compounds, such as benzene verse benzenethiol, ${ }^{123}$ observation of surface enhancement where plasmons are weak, such as on $\mathrm{Pt},{ }^{124}$ and direct EELS measurement of low energy charge transfer bands of molecules chemisorbed to Ag. ${ }^{125}$

It has been suggested that charge-transfer states formed by the chemisorption by some compounds to the metal surface can yield Raman enhancement on the order of 10-100 providing these charge transfer bands are at least partially resonant with incident light. ${ }^{126}$ This magnitude of enhancement is not observed on all metals however, and thus coupling with a plasmon is required for the necessary enhancement. ${ }^{122}$ It has been shown theoretically that the coupling of a plasmon mode to the charge transfer events can increase the Raman cross section of certain vibrational modes. ${ }^{123}$ While the necessity of the chemical enhancement mechanism to explain 
single molecule SERS has been debunked, ${ }^{122}$ these effects are still observable and may yet contribute notable increases to enhancement of Raman scattering and are important to consider in the design SERS experiments and application.

\subsubsection{Resonance Raman Effects}

What is often attributed to another source of chemical enhancement is the so-called resonance-Raman effect that occurs when the incident radiation is in resonance with an electronic transition of the molecule. Thus popular SERS labels, such as Rhodamine 6G, are often fluorophores, as matching the exciting light source with the absorption band of the fluorphore leads to very intense SERS. ${ }^{120}$ This effect results in a naturally large Raman cross section of the molecule, ${ }^{127}$ even in solution, and should not be confused with the surface enhancement effect. $^{122}$ This may not be apparent in solution measurements as the strong fluorescence of such compounds may make Raman spectra difficult to observe, unless the fluorescence is quenched, which tends to happen when the molecule is adsorbed onto a metal surface. ${ }^{128}$ So while molecules such as Rhodamine $6 \mathrm{G}$ do make excellent reporters in SERS, their naturally high Raman cross section should not be confused with an enhanced cross section. In fact this very error was made when Nie and Emory first reported single molecule SERS, as they did not calculate the SERS EF by the current conventional means but by estimating the SERS cross section against a typical Raman cross section on the order of $10^{-30} \mathrm{~cm}^{2}$ as opposed to the $10^{-24} \mathrm{~cm}^{2}$ cross section of Rh6G. ${ }^{120,110}$ This places the actual enhancement factor of this experiment close to $10^{8}$ as opposed to $10^{14}$, indicating the EM mechanism of enhancement alone is in fact sufficient for single molecule detection. ${ }^{122,110}$ 


\subsection{Concluding Remarks}

The extensive review of plasmonic interactions in this section was necessary to demonstrate the complexity of interactions available in nanocube ensembles used in this work. The future chapters will attempt to rationalize observations obtained from optical characterization and SERS experiments using the plasmonic interactions presented here. Due to the shear multitude of possible plasmonic interactions that can occur in these systems, it can be difficult to pin down the source of any observation to be the result any singular cause.

The experimental techniques used in this work have been described here in detail. The control that can be attained in the synthesis of silver nanocrystals is considerably advanced, so this work uses the established techniques to design and utilize nanocrystals with our choice of size and shape. In particular the focus will be on silver nanocubes of $\sim 40 \mathrm{~nm}$ in edge length. This size and geometry was chosen as it is an easy to produce, stable nanocrystal that displays multiple plasmonic modes of different order, but the resonances are still sharp, distinguishable and thus easy to interpret. The assembly of these nanocubes is directed through a number of means to study the interactions between each other and their substrate, and likewise how these interactions affect one another.

Lastly the multitude of effects that result in the surface-enhancement of Raman spectroscopy is examined. In this work SERS is mostly used as a tool to probe the near-field properties of these nanocube assemblies. Thus an understanding of how certain properties of the nanocrystals, their assembly, and the molecules being observed with SERS is a natural requirement to interpret the results. 


\section{Strongly Interacting Silver Nanocubes on Silicon Substrates}

It had been previously demonstrated that by modulating the thickness of a thin film of material with a high refractive index, for example silicon, excellent control over the plasmonic properties of supported silver nanocubes could be achieved. ${ }^{50,51}$ The ability to shift select plasmon resonances to a new position proved to be useful in increasing the sensitivity of the hybridized quadrupolar mode to the surrounding environment. The nanocube proved to be an ideal geometry for this application due to strong interactions with the surface, the presence of multiple orders of LSPR modes, and the ability to spatially separate them. ${ }^{52}$ It was then speculated that this ability to tune the plasmon resonances may in fact prove to benefit another rapidly advancing and popular plasmonic-based sensing platform; surface-enhanced Raman spectroscopy. The hypothesis was that by shifting the plasmon resonances of an existing system it may be possible to put them in-line with Raman excitations and emissions, yielding significant enhancement over systems where the resonances are not-so well aligned. Initial results suggested this just might be the case, ${ }^{51}$ so an in-depth thorough investigation into the matter was launched. $^{129}$

This chapter will describe the effects on optical properties of silver nanocubes as their interaction with the substrate, and with each other are modulated. These properties are tuned by the Langmuir-Blodgett deposition of nanocubes at various lateral surface pressures, to control the degree of inter-particle coupling, and deposited on various thicknesses of silicon thin film, to tune the interaction with their substrate. SERS is used as a tool to probe the near-field optical properties, to determine if there is merit in this approach for SERS applications. 


\subsection{Experimental}

\subsubsection{Synthesis of Silver nanocubes}

Materials: Silver nitrate $\left(\mathrm{AgNO}_{3}, 99+\%\right)$, polyvinylpyrrolidone (PVP, $\mathrm{Mw} \approx 55000$ ), anhydrous $99.8 \%$ ethylene glycol (EG), $\mathrm{Na}_{2} \mathrm{~S}$ and $\mathrm{NaCl}$ were purchased from Sigma-Aldrich and used as obtained.

Silver nanocubes were synthesized based on a modified approach to established methods. ${ }^{130}$ In a typical synthesis $35 \mathrm{~mL}$ of ethylene glycol (EG) was heated to $150^{\circ} \mathrm{C}$ in a $100 \mathrm{~mL}$ round bottom flask and allowed to heat for $1 \mathrm{~h}$. Syringes were then used to inject $2.5 \mathrm{~mL}$ of $0.8 \mathrm{mg} / \mathrm{mL}$ polyvinylpyrrolidone (PVP, Mw 55 000, Sigma Aldrich) solution. After 2 minutes, 200 $\mu \mathrm{L}$ of $3 \mathrm{mM}$ sodium sulfide and after an additional 2 minutes $1.5 \mathrm{~mL}$ of $282 \mathrm{mM}$ silver nitrate was injected dropwise (at $0.5 \mathrm{~mL} / \mathrm{min}$ if by syringe pump). The reaction was monitored by UV-Vis spectroscopy, periodically taking small aliquots out of the reaction flask and dispersing in an ethanol-filled cuvette. The reaction was typically quickly seeded by small silver nanoparticles, characterised by a dark brown collar and a resonance $\sim 400 \mathrm{~nm}$, which rapidly grew after about $10-15$ minutes. The reaction was quenched in an ice-bath after the appearance of a distinct mode at $350 \mathrm{~nm}$ and when the dipole had reached 430nm (10-20 minutes). This synthesis is scalable to some degree, and was often performed at double scale, simply by doubling the volume of EG and of the injected reagent solutions.

Upon cooling, the reaction flask was diluted with ethanol, and the sample was centrifuged, redispersed in ethanol, and further centrifuged several times at $13000 \mathrm{~g}$ 's. After 5 rinses the sample is stored in ethanol. 


\subsubsection{Langmuir - Blodgett Silver Nanocube Monolayers}

Materials: 1,2-Dipalmitoyl-sn-glycero-3-phosphoethanolamine-N-(lissamine rhodamine B sulfonyl) (ammonium salt, DPPE-RhB) and 1,2-dioleoyl-sn-glycero-3-phosphocholine (powder, DOPC) were purchased from Avanti Polar Lipids as a powder and used as obtained. Silicon thin films were fabricated by electron beam physical vapour deposition (PVD) on to glass by the Carleton University Microfabrication Facility. The silicon thin-film substrates were stored in a container under ambient conditions until use. Prior to deposition of the nanocube monolayers, the substrates were rinsed in methanol under sonication for 10 minutes.

A Nima 311D Langmuir-Blodgett trough filled with Milli Q water $(18.2 \mathrm{M} \Omega \bullet \mathrm{cm})$ was used to prepare the silver nanocube monolayers. A sample of silver nanocubes was re-dispersed in $200 \mu \mathrm{L}$ of chloroform. These samples originate from an ethanol-suspended $2 \mathrm{~mL}$ sample displaying an extinction of 0.250 to 0.500 at the maximum of the dipolar peak $(430-445 \mathrm{~nm})$, corresponding to an estimated $\sim 10^{12}$ nanocubes. To the $200 \mu \mathrm{L}$ sample of nanocubes, an amount of dioleoylphosphatidylcholine (DOPC) was added to act as a passive lipid spacer and to promote stability of the cubes at high surface pressures. This amount was frequently adjusted between experiments, but was typically between $2 \mathrm{uL}$ to $10 \mathrm{uL}$ of $1 \mathrm{mg} / \mathrm{mL}$ DOPC in ethanol. It was found a value of about 1000 - 2000 lipid molecules per nanocube was ideal for promoting the desired effects while not significantly altering the pressure/area isotherm and monolayer behaviour. In addition to the lipid spacer, a Rhodamine B labelled lipid (DPPE-RhB), was sometimes used as Raman label for SERS experiments. Again the amount of this varied between experiments, the details of which will be discussed later, the ratio of DPPE-RhB/DOPC added in this experiment was $10 \%$ by mass. 
The nanocube-lipid solution was deposited onto the water surface using a microsyringe dropwise, and left for 20 minutes to allow for the chloroform to evaporate. A pressure/area isotherm was run by allowing the barrier to compress the layer at $40 \mathrm{~cm}^{2} / \mathrm{min}$, and multiple compression/expansion cycles were run for the purpose of annealing the monolayer. The monolayers were deposited onto substrates by vertical dipping, retracting the substrate at $2 \mathrm{~mm} / \mathrm{min}$. The substrates were glass slides half-coated with a thin film of silicon. Since the substrates were stored under ambient conditions, an oxide layer had formed to make the surface chemistries of each side similar allowing for the deposited monolayer to be identical in density on each surface. The deposited monolayers were removed from the back side of the substrate via mechanical abrasion by a methanol-soaked wipe. The samples were stored in a container under ambient conditions.

\subsubsection{Characterization}

UV-Vis extinction spectra of the colloidal nanocubes in ethanol and of deposited silver nanocube monolayers were recorded using a Shimadzu UV-2450 UV-Vis spectrophotometer. Baseline spectra of the underlying substrate, such as the silicon thin film, were subtracted off the extinction spectra of the AgNC monolayers. Topography measurements of the deposited monolayers were done using Ntegra (NTMDT) atomic force microscope in semi contact mode in air at $23^{\circ} \mathrm{C}$ with $512 \times 512$ points per image. A $100 \times 100 \mu \mathrm{m}^{2}$ scanner (Ntegra) and silicon cantilevers with a reflective gold coating were used $(1.75 \mathrm{~N} / \mathrm{m}$ spring constant, $70 \mathrm{kHz}$ resonant frequency, NSG03, NT-MDT). Transmission Electron Micrographs were collected on a FEI Tecnai G2 F20 TEM at the Carleton University Nano-imaging Facility. 


\subsubsection{Surface - Enhanced Raman Spectroscopy}

Surface enhanced Raman spectra were collected using a single grating monochromator (Jobin Yvon, focal length $640 \mathrm{~mm}, 1600$ lines/mm) with a liquid N2 cooled CCD camera (Princeton Instruments). Excitation was done using an $\mathrm{Ar} / \mathrm{Kr}$ ion laser (Coherent) at wavelengths 458nm, $488 \mathrm{~nm}, 514 \mathrm{~nm}, 568 \mathrm{~nm}$, and $647 \mathrm{~nm}$. For each measurement 3 spectra were taken with a $30 \mathrm{~s}$ accumulation time, and each measurement was repeated on $3-9$ different areas of each substrate. SERS spectra were collected by WinSpec/32 software, processed in GRAMS/AI spectral data processing software, and cyclohexane was used for Raman shift calibration.

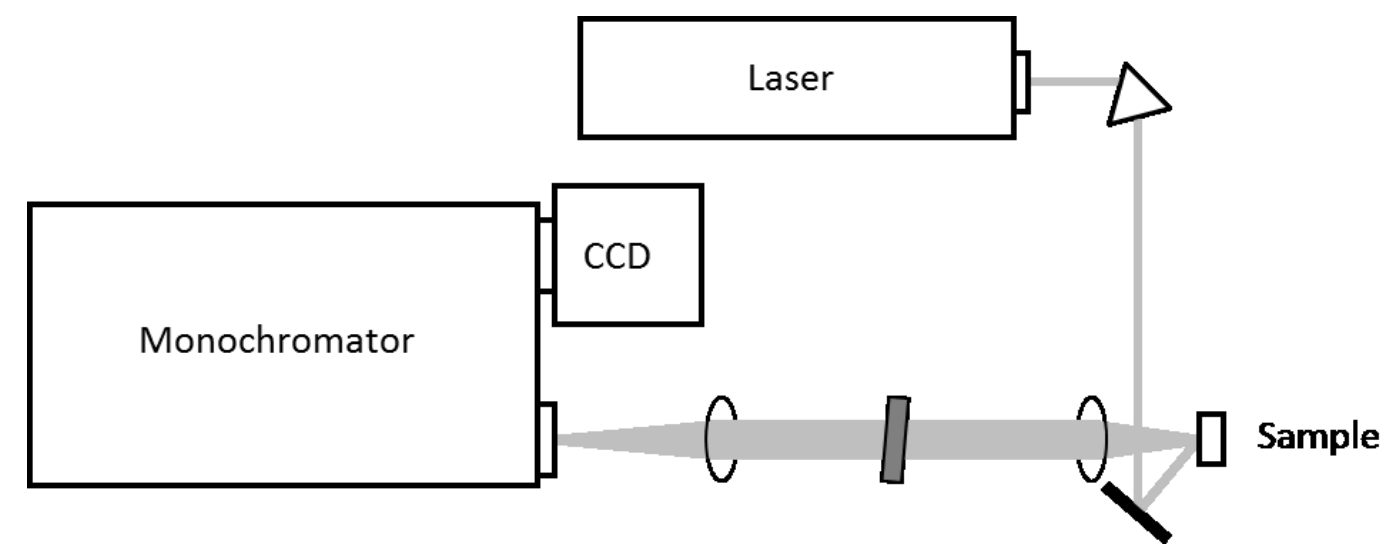

Figure 2.1- Schematic of the Raman spectrometer used in this experiment. The sample is placed on a 3-axis translatable stage. Collection is performed by $45^{\circ}$ scattering relative to the exciting beam. Spectra are collected using a single grating monochromator with a focal length of $640 \mathrm{~nm}$ and a liquid $\mathrm{N}_{2}$ cooled CCD. 


\subsection{Results and Discussion}

\subsubsection{Optical Properties of Strongly Interacting Nanocubes on Silicon Substrates}

Silver nanocubes with an edge length of $40 \mathrm{~nm}$ were prepared for this experiment, confirmed by AFM topography. The extinction spectrum of the colloid is presented in Figure 2.2 and is characterized by a dipolar mode at $440 \mathrm{~nm}$, a characteristic octupolar mode at $350 \mathrm{~nm}$, and in this instance the nanocubes are of sufficient size for a "bright" quadrupolar mode at $\sim 390 \mathrm{~nm}$.

A

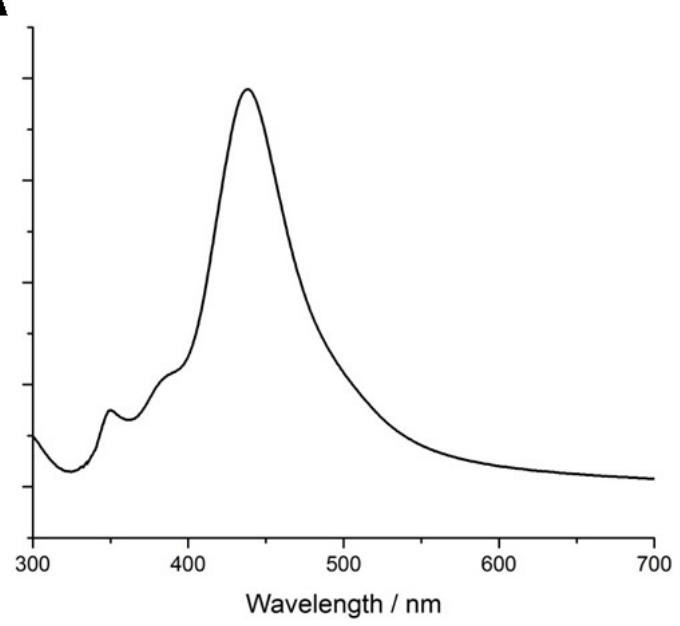

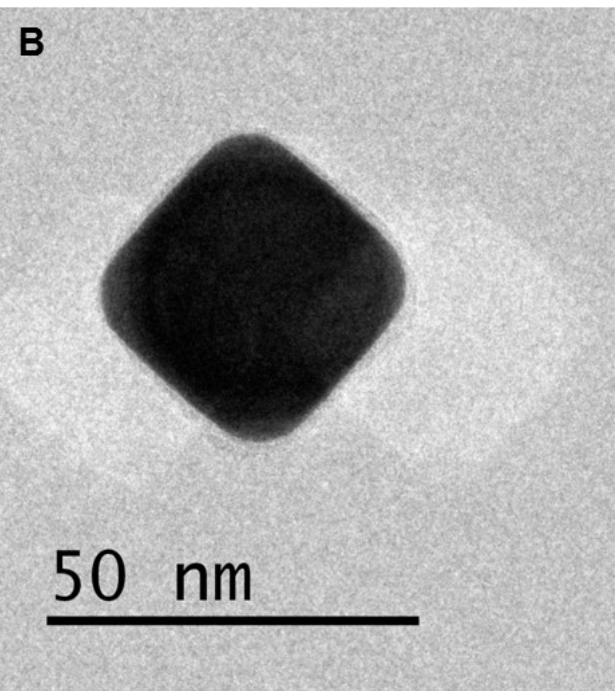

Figure 2.2 - A) UV/Vis extinction spectrum of colloidal 40nms nanocubes suspended in ethanol and B) a transmission electron micrograph of an individual nanocube

Silver nanocube monolayers were deposited on glass slides which were half coated with a $25 \mathrm{~nm}$ silicon thin film. The monolayers were deposited on several slides, each at a different lateral surface pressure. Langmuir-Blodgett monolayers tend to have excellent long-range order, so the monolayer deposited on both the glass and silicon thin film were consistent. The presence of a native oxide layer on the silicon thin films ensure the surface chemistries are similar enough to facilitate this near-identical deposition. The deposition of comparable monolayers on both surfaces was confirmed by observation of similar particle densities by AFM. These films were deposited at $0.3 \mathrm{mN} / \mathrm{m}, 1 \mathrm{mN} / \mathrm{m}, 4.5 \mathrm{mN} / \mathrm{m}, 7 \mathrm{mN} / \mathrm{m}$, and $20 \mathrm{mN} / \mathrm{m}$. The topology of $1 \mathrm{mN} / \mathrm{m}$ 
monolayers are displayed in Figure 2.3. As the surface pressure increases the interparticle spacing between nanocubes in these monolayers decreases drastically until a near-solid layer of nanocubes is presented at the highest pressures. The degree of aggregation also increases at the solid pressures. At about $20 \mathrm{mN} / \mathrm{m}$ the collapse point of the monolayer was reached, leading to high levels of aggregates and multilayer stacking of nanocubes.
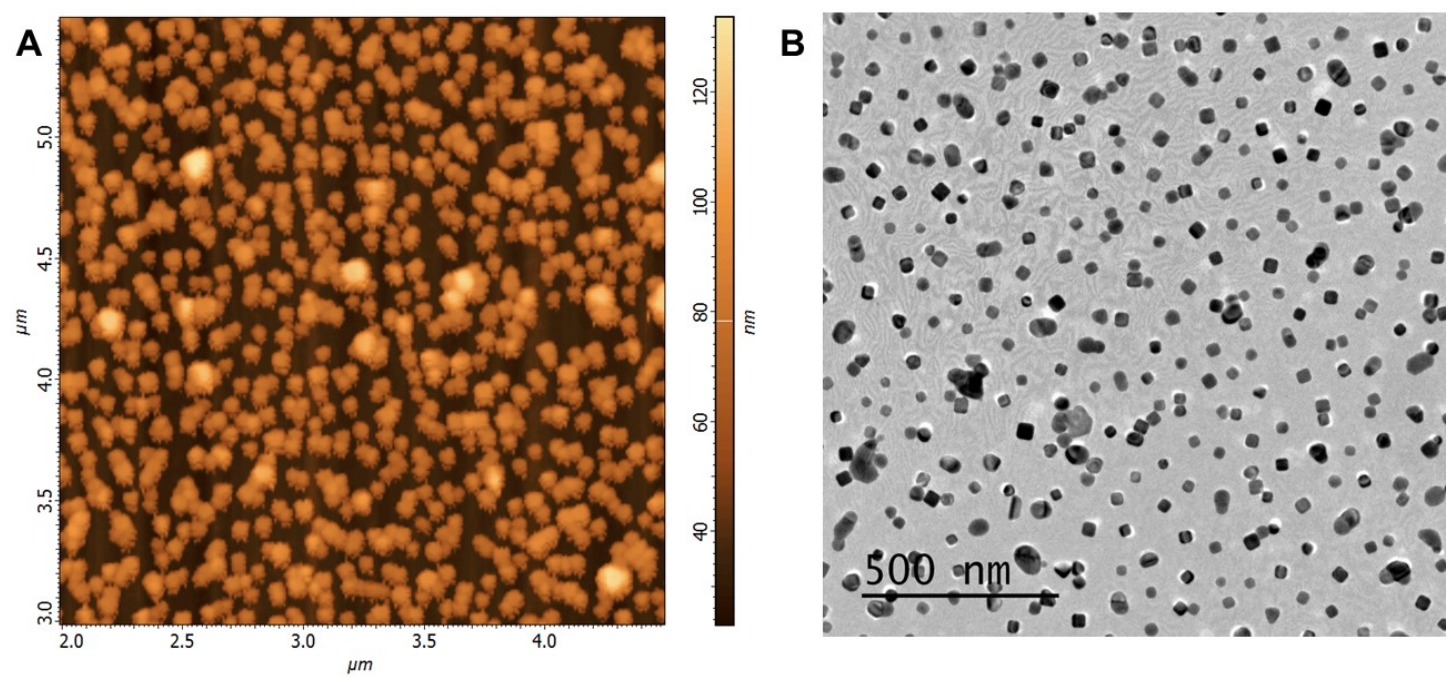

Figure 2.3 - A) Atomic force microscopy low-pressure $(1 \mathrm{mN} / \mathrm{m})$ Langmuir-Blodgett monolayer of 40nm silver nanocubes deposited on glass B) TEM image of a similar monolayer of 30nm silver nanocubes deposited at $1 \mathrm{mN} / \mathrm{m}$ on a TEM grid.

The extinction spectra on glass in Figure 2.4A are characterized by the presence of the dipolar peak at $405 \mathrm{~nm}$, a quadrupolar mode at $380 \mathrm{~nm}$ and a higher order mode characteristic of nanocubes at $350 \mathrm{~nm}$. As the surface pressure of the monolayers increase, transverse plasmonic interactions broaden the D and Q modes until they are indistinguishable and the onset of dipoledipole coupling can be observed in a large band in the red region of the spectrum. The D-D coupling band, at $\sim 600-700 \mathrm{~nm}$, redshifts with increasing interaction between the nanocubes, until reaching the highest pressure substrate at $20 \mathrm{mN} / \mathrm{m}$. This substrate displays a sudden $40 \mathrm{~nm}$ blue-shift of the D-D coupling mode indicating a possible weakening of the coupling interaction despite the increased density of the monolayer. It is speculated that these monolayers contain sites of conductive contact or sufficiently low interparticle spacing to allow for quantum 
tunneling between nanocubes. Indeed the presence of sub-nanometer gaps have been observed in similar monolayers, as shown in Figure 2.4B. The increase in population of these gaps at high pressures may explain the blue-shifting of the coupling mode and reduced E-field enhancement observed in these monolayers. Additionally, as will be displayed in further detail in the following chapter, sintering and coagulation of the nanocubes is possible at high pressure LB monolayers, further complicating interpretation of this result. One of the initial goals of the work had been to detect quantum-tunneling in an ensemble measurement of the nanocubes, however it is difficult to differentiate between interacting cubes in the quantum regime and conductive contact regime in the ensemble.
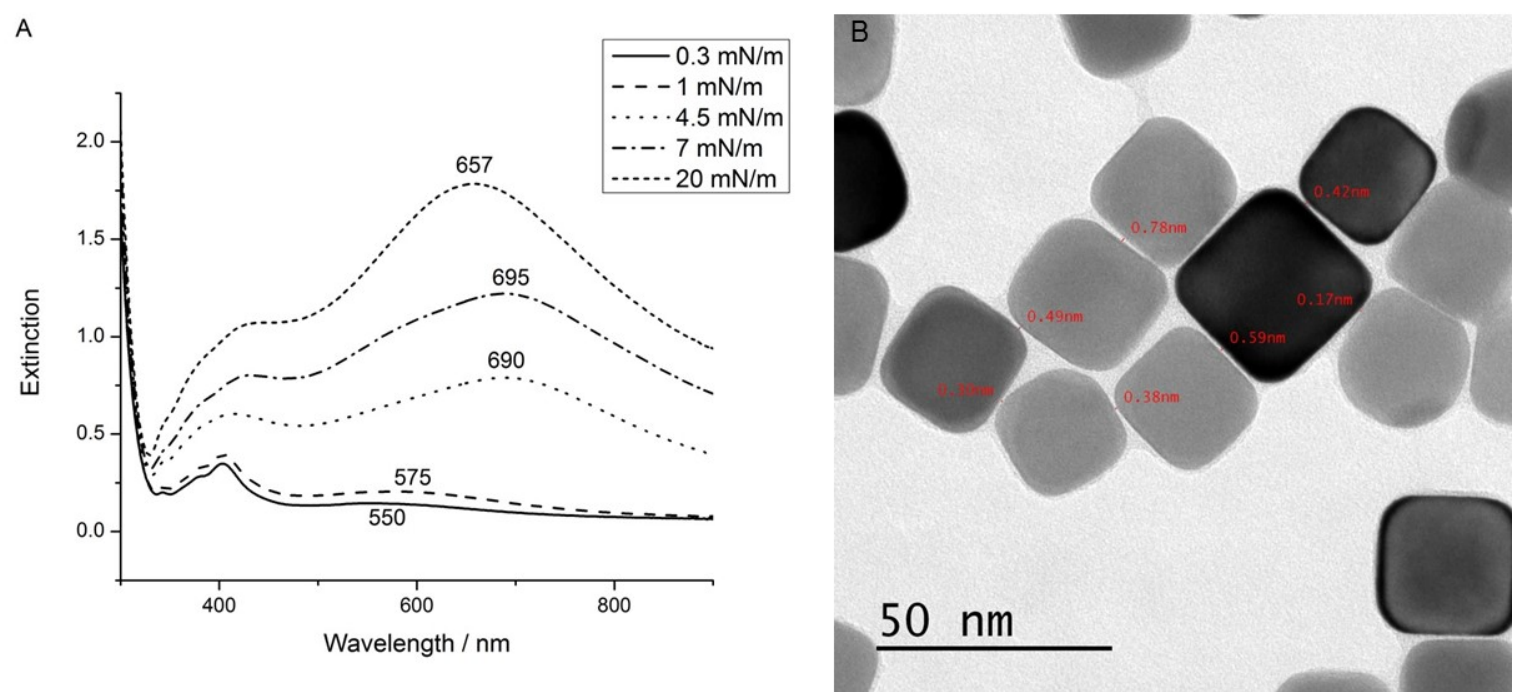

Figure $2.4-$ A) UV/Visible extinction spectra on glass of silver nanocube monolayers deposited at various surface pressures. As the particle density and hence average interparticle distance increases a red-shift in the D-D coupling band (longest wavelength peak) is observed. At the highest density monolayer, a blue-shift in the spectrum is observed. B) TEM image from a similar set of monolayers, exhibiting sub-nanometer junctions and conductive contact. These narrow junctions may be responsible for the blue-shift observed in the highest density monolayer which is a result of charge-transfer processes between adjacent nanoparticles.

The extinction spectra on $25 \mathrm{~nm} \mathrm{Si}$, shown in Figure 2.5 , are in many ways similar to those on glass, but with some notable differences. As one would expect, the substrate induced hybridisation of the plasmon modes causes splitting of the D and Q modes, blue-shifting and redshifting them respectively. Furthermore, the bonding dipolar plasmons are blue-shifted and 
narrowed with respect to their counterparts on glass. Lastly the blue-shift of the D-D mode observed at $20 \mathrm{mN} / \mathrm{m}$ on glass does not occur on Si.
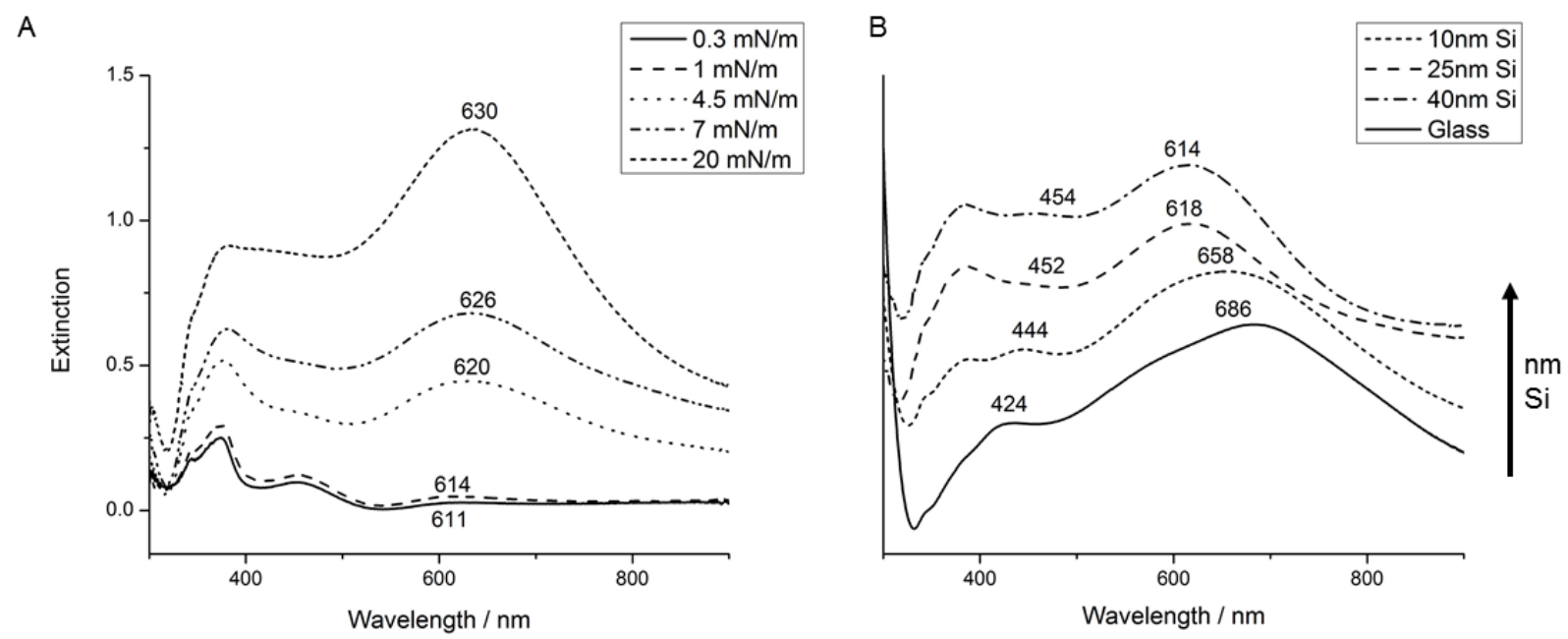

Figure 2.5 - A) UV/Visible spectroscopy of silver nanocube monolayers deposited on silicon at various surface pressures. A continuous red-shfit of the D-D coupling mode is observed as the AgNC density increases. These peaks are significantly blue-shifted and narrowed with respect to their glass counterparts in Figure 2.4A B) UV/Visible spectroscopy of silver nanocube monolayers deposited on varying thicknesses of silicon. As the thickness of the silicon thin film is increased, the D mode red-shifts but the D-D mode blue-shifts and narrows.

The effects of the silicon substrate were further explored by depositing $4 \mathrm{mN} / \mathrm{m}$ monolayers on silicon thin films of varying thickness. Figure 2.5 displays the resultant UV-Vis extinction spectra. As the thickness of Si increases, the D-D coupling band is blue-shifted and narrowed. This is counter-intuitive to the common trend of dipolar LSPRs red-shifting with increased refractive index of the environment. These observations are believed to be due to the presence of a substrate-mediated charge transfer process. ${ }^{131}$ Such a process would explain the blue-shift of the bonding dipolar mode, reduced E-field enhancement noted in the following section, and the negligible effect of charge transfer processes previously observed in the $20 \mathrm{mN} / \mathrm{m}$ monolayer as this is suspected to already be occurring. 


\subsubsection{Surface-Enhanced Raman Spectroscopy}

Surface enhanced Raman spectroscopy was used as a tool to probe the electric field enhancements yielded by the previously described silver nanocube monolayers. SERS was done at $458 \mathrm{~nm}, 488 \mathrm{~nm}, 514 \mathrm{~nm}, 568 \mathrm{~nm}$, and $647 \mathrm{~nm}$ to probe resonances with the nanocube dipole absorption, Raman active label absorption (Rhodamine B, $\lambda \max =543 \mathrm{~nm}$ ), and the dipole-dipole coupling modes.

The obtained SERS spectra are shown in Figure 2.6, The two peaks used to compare SERS results are those at $1650 \mathrm{~cm}-1$, a $\mathrm{C}=\mathrm{C}$ stretch of Rhodamine $\mathrm{B}$, and at $1765 \mathrm{~cm}-1$ a $\mathrm{C}=\mathrm{O}$ stretch of PVP which typically does not appear in solution spectra. ${ }^{132}$ These analytes differ largely in their position in the substrate. DPPE-RhB was incorporated into the monolayer, and as such would lie at the surface of the substrate, between the nanocubes, while PVP, as the capping agent of the nanocubes, is bound to their surface. As such the goal was to compare trends in the surface enhanced spectra and elucidate location specific near-field properties.

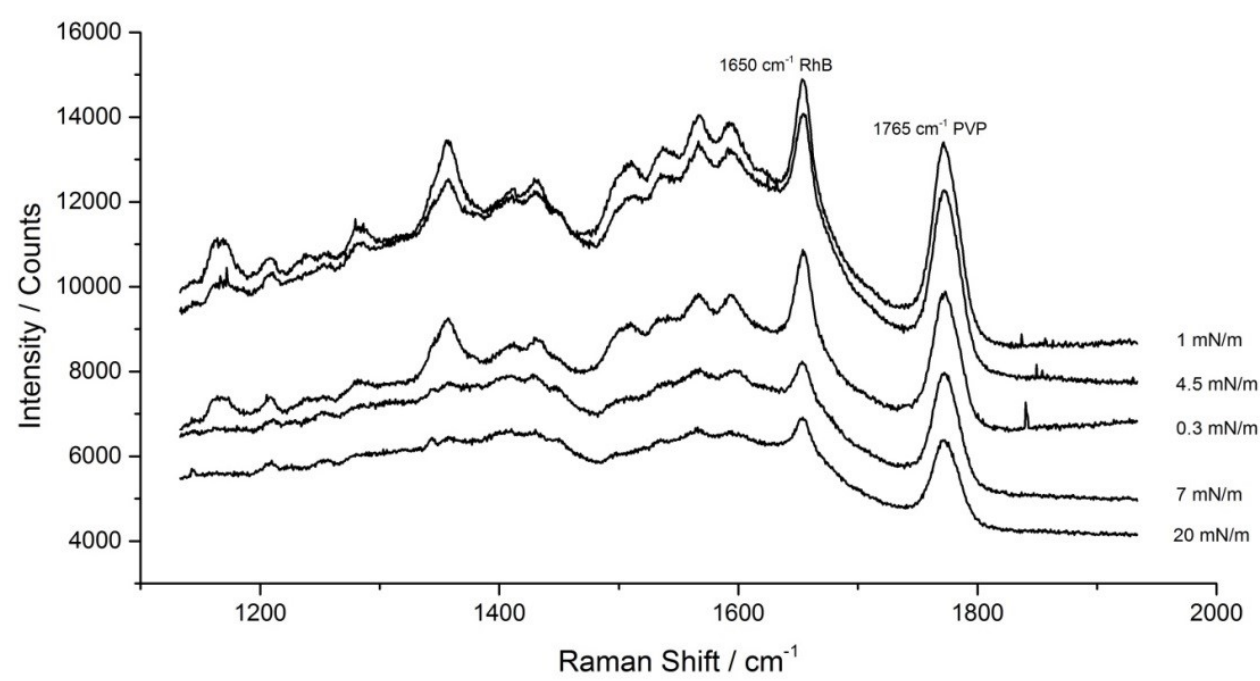

Figure 2.6 - SERS Spectra of Rhodamine B and PVP obtained in this experiment with 458nm excitation. The main probe of the experiment was the ring-breathing mode of $\mathrm{RhB}$ at $\sim 1650 \mathrm{~cm}^{-1}$, though the $\mathrm{C}=\mathrm{O}$ stretch of $\mathrm{PVP}$ at $\sim 1765 \mathrm{~cm}^{-1}$ was also examined. DPPE-RhB was incorporated into the monolayer while PVP was the native capping agent of the nanocubes. 
The initial expectation was that greatest SERS enhancement would occur when the monolayer was as compressed as possible as this would minimize interparticle distance and optical extinction. ${ }^{133}$ The results displayed in Figure 2.7 indicate that this is not the case. At shorter excitation wavelengths $(458 \mathrm{~nm}, 488 \mathrm{~nm}, 514 \mathrm{~nm})$, liquid phase substrates $(1 \mathrm{mN} / \mathrm{m})$ demonstrated maximal SERS enhancement on glass. At $458 \mathrm{~nm}$, this can explained by the excitation wavelengths matching with the individual dipolar mode plasmons and not exciting the coupling modes responsible for the enhancement at higher particle densities. At slightly longer wavelengths this may be due to populations of weakly interacting clusters and aggregates, which would provide the necessary sources of enhancement in this spectral region. The higher pressure monolayers are dominated by strongly interacting nanocubes, which resonate at longer wavelengths. As such the SERS intensity at with excitation wavelengths at $568 \mathrm{~nm}$ and $647 \mathrm{~nm}$ is maximised on the $4.5 \mathrm{mN} / \mathrm{m}$ sample. Resonance of the excitation and Raman emission with the bonding dipolar mode leads to increased enhancement, however despite similar resonances existing for the highest pressure monolayers, they do not exhibit the same degree of enhancement. The reduction in enhancement at $20 \mathrm{mN} / \mathrm{m}$ can be explained by the onset of charge transfer processes mediated by the substrate. ${ }^{134}$ This explanation is supported by the far-field optical properties of the sample, displaying a blue-shift and narrowing of the plasmon. These charge transfer effects are not observed optically with the $7 \mathrm{mN} / \mathrm{m}$ monolayer however, which displays significantly reduced enhancement in comparison to the $4.5 \mathrm{mN} / \mathrm{m}$ slide. The reason for the reduced enhancement in this case is likely due to the dispersion of the electric field enhancement experienced in large particle clusters or aggregates. ${ }^{135}$ This sample contains a nearcontinuous layer of nanocubes, which would reduce the total electric field enhancement at hotspots due to the increased average cluster size in comparison to lower pressure monolayers. The 
$4.5 \mathrm{mN} / \mathrm{m}$ shows an ideal balance between minimal interparticle distance and average cluster size in this set of substrates.
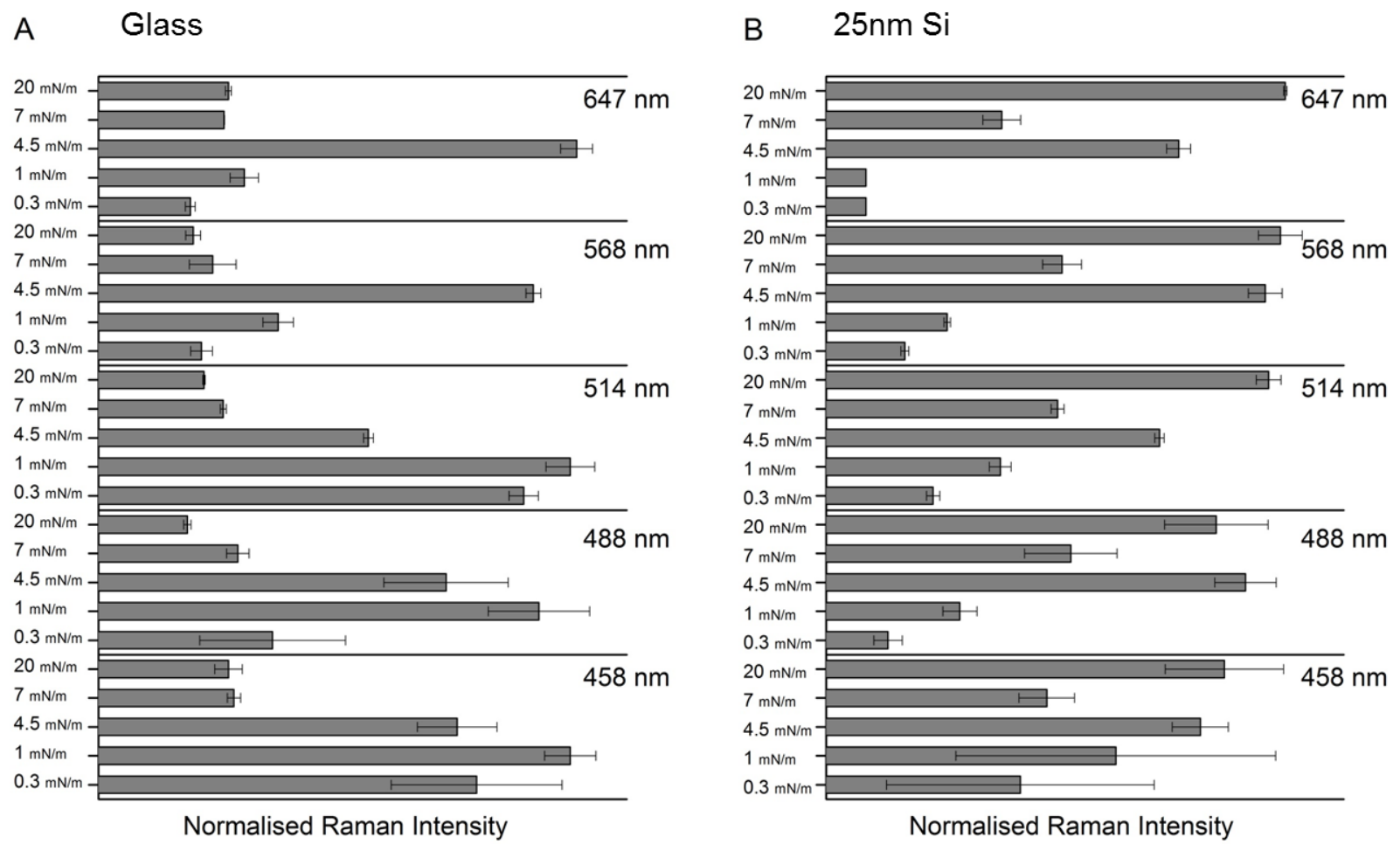

Figure 2.7 - Histograms of normalised average intensity of Raman scattering observed at each excitation wavelength. Depending on excitation, monolayers on glass show the best enhancement at lower particle densities while those on silicon favour the highest particle density.

The pressure-dependent enhancement trends are considerably different on silicon than on glass. At all excitation wavelengths, the $4.5 \mathrm{mN} / \mathrm{m}$ and $20 \mathrm{mN} / \mathrm{m}$ substrates display maximum enhancement. As on glass, the $4.5 \mathrm{mN} / \mathrm{m}$ monolayer display that intermediate particle density is ideal for successful SERS enhancement. With increasing pressure $(7 \mathrm{mN} / \mathrm{m})$ the SERS intensity once again drops. Unlike glass however, the $20 \mathrm{mN} / \mathrm{m}$ display great enhancement, on par or greater than that displayed by the $4.5 \mathrm{mN} / \mathrm{m}$ substrate. As mentioned before, the $20 \mathrm{mN} / \mathrm{m}$ substrate was deposited at the collapse point, and contains some three dimensional order of the nanocubes. Thus the results indicate the underlying silicon substrate inhibiting electric field enhancement at the surface since 1) The multi-layered substrate exhibits greater enhancement 
since the interaction with the surface is minimized and 2) the low pressure $(1 \mathrm{mN} / \mathrm{m})$ monolayer does not display the same relatively high enhancement as displayed on glass.

SERS enhancement was found to be significantly weaker on silicon when compared to identical monolayers on glass substrates. The Raman intensity was found to be between $2-23$ times lower on silicon than glass, largely dependent on the monolayer's particle density. The trend demonstrated in Figure 2.8 displays how as the particle density of the monolayer increases, the disparity between the SERS signals on glass versus silicon decreases. At $20 \mathrm{mN} / \mathrm{m}$ the intensities are almost equal, with the glass signal ranging between 1 to 2 times that recorded on silicon, increasing respectively with the wavelength of the excitation source. This clear trend indicates that as the particle density increases the importance of the substrate mediated interactions also decrease. As the interaction between nanoparticles becomes stronger, the mixed modes formed by the inherently weaker plasmon-substrate interactions become supressed.
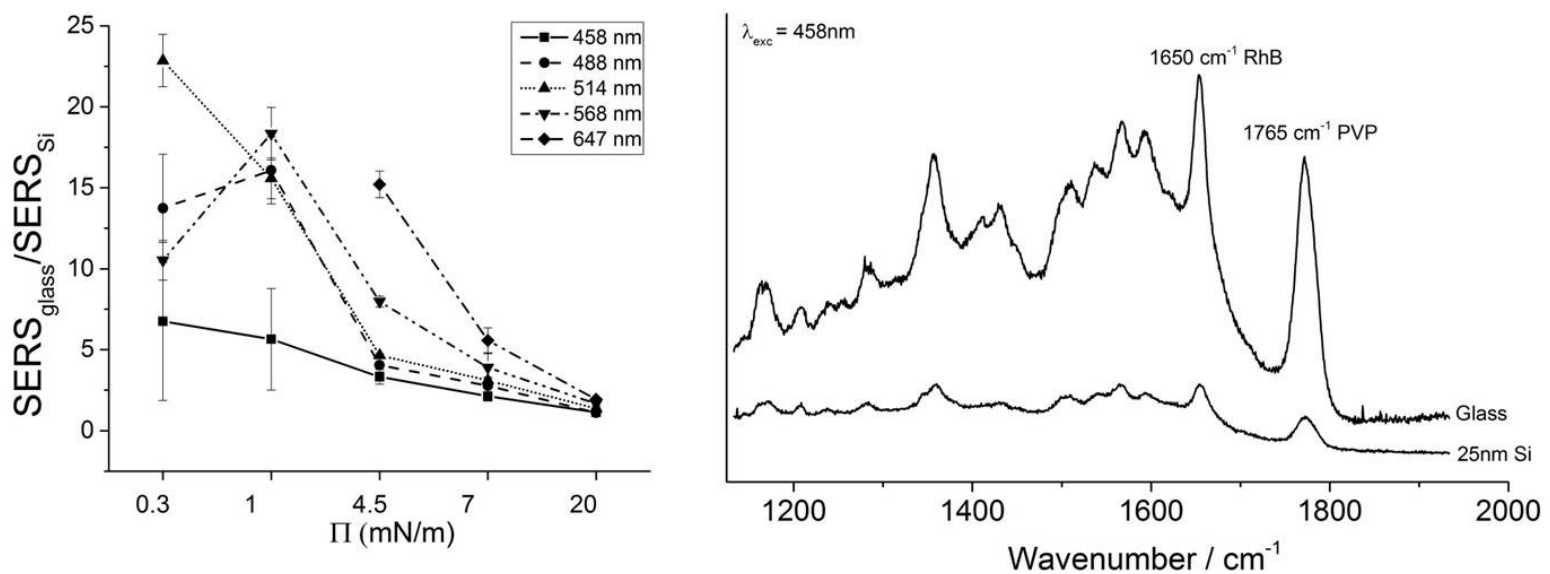

Figure 2.8 - A) Comparison of the intensity of SERS on glass against on $25 \mathrm{~nm}$ silicon thin film. SERS intensity on the Si film was far lower than on the glass. As the particle density increases the SERS-inhibiting substrate effects of the silicon become less predominant B) SERS Spectra recorded at $458 \mathrm{~nm}$ of a AgNC monolayer deposited at $4.5 \mathrm{mN} / \mathrm{m}$. The intensity on glass is about $5 \mathrm{x}$ greater than that on $25 \mathrm{~nm} \mathrm{Si}$ 
To further probe the substrate effect, both the SERS activity of Rhodamine B and PVP were compared. While not being directly comparable due to much different surface concentrations and Raman cross sections, evaluation of the trends that have been discussed to this point but with respect to PVP intensities may have yielded some further insight into the nearfield properties of the particles. The pressure and substrate-dependent trends, however, were found to be nearly identical to those observed with DPPE-RhB. The PVP signal also became significantly weaker than $\mathrm{RhB}$ as the excitation was moved into the red as it does not benefit from the resonant-Raman effects.

\subsubsection{Aging of the Silicon Substrates and Re-evaluation of Some Observations}

The blue-shift in the dipolar coupling mode on silicon observed previously in this chapter, visible clearly in Figure 2.5 was initially believed to be the natural result of supporting a coupling mode on a high refractive index substrate. Indeed results similar to these were reproduced consistently for some time. However after some time it was noted that the LSPR peak shift were behaving less predictably in deposited monolayers on silicon thin films. Figure 2.9 displays an example of this. The dipolar mode does not redshift monotonically with increasing silicon thickness; in fact it seems to show the reverse trend. Meanwhile the bonding dipolar mode shifts erratically, and important to note is that in this case it is red-shifted relative to glass, not blue-shifted. 


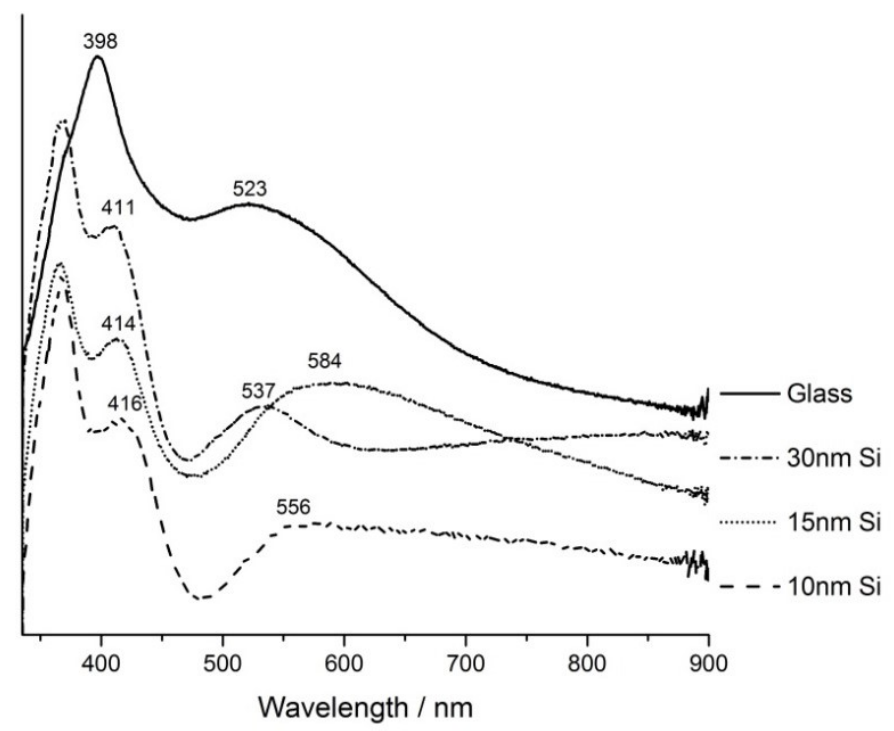

Figure 2.9 - Silver nanocube monolayers deposited at $3 \mathrm{mN} / \mathrm{m}$ on varying thicknesses of $\mathrm{Si}$ after the slides had been aged in air for several months. The plasmon shifts behaved erratically and did not behave as established in previous experiments

It became clear that the silicon thin films used in previous experiments did not age well. While it had initially been assumed a consistent, passivating oxide was formed on the silicon surface, it became apparent the oxide content in the films was not consistent. It is believed that as the source of the silicon thin film is physical vapour deposition (PVD), the amorphous silicon film may have contained pits and pores which would have enabled native oxide growth to dig into the films. This would have resulted in inconsistent oxide content in the films, and gives an explanation for the erratic results such as those observed in Figure 2.9. Due to the inconsistency in results that were yielded with the aging silicon thin films, and the indication of results that high refractive index oxides my yield different optical properties than those presented in this chapter, future work used a different source for substrate materials. In Chapter 4 the optical properties of strongly coupled silver nanocubes on titanium oxide thin films will be examined in detail. 


\subsection{Conclusions}

This work displayed the ability to tune plasmon resonances using silicon thin films. Monolayers deposited on these films however displayed weaker E-field enhancement than those on a standard, low refractive index dielectric, such as glass. Furthermore, the red-shift as opposed to a blue-shift in the D-D modes observed in later experiments challenges posed the question of the original source of this observation. Conventional knowledge predicts a red-shift with increasing refractive index of the surrounding environment in most plasmonic modes, and the narrowing and blue-shifting of the plasmon is indicative of charge-transfer effects. While the semi-metal nature of silicon could surely facilitate such a charge transfer, how was this being performed in the presence of the native oxide at its surface? This perhaps could be achieved by plasmon-exciton coupling, ${ }^{136}$ or some other exotic effect. However the origin of these observations remains unexplained, and merit further investigation. 


\section{Lessons in the Assembly of Silver Nanocubes}

This overall aim of this work is to study the near-field and far-field optical properties of ensembles of nanocubes, and to do so requires some degree of control over the assembly of the nanocubes. The target features aimed for in the design of these ensembles were to control the interparticle distance in a monolayer assembly with excellent long range order. The optical properties also must be distinct and discernable, so to this end nanocubes were fabricated to have as sharp a resonance as possible, which typically entails minimization in size. The target interparticle distance was intended to be as small as possible, which for Langmuir-Blodgett assemblies entailed compression to high lateral pressures. Unfortunately a unique problem arose with the attempt to fabricate monolayers in such a manner, heavy aggregation and coalescence of the nanoparticles. Indeed some monolayers that were deposited on a TEM grid almost appeared to have melted at the air water interface. The unique surface interactions in such a system are explored in this section.

It had also become apparent after some time that while the Langmuir-Blodgett technique offers excellent control over particle density, it cannot reliable tune interparticle distances. Other assembly strategies were thus explored revolving around the self-assembly of the nanocubes. While in the end these strategies were not employed, valuable lessons were learnt in the attempt which may be of use in the future of directed assembly of nanocube junctions. 


\subsection{Coalescence of Silver Nanocubes in Langmuir-Blodgett Monolayers}

It was noted on several occasions that on compression of the Langmuir-Blodgett monolayer, an irreversible colour change to a grey colour from the typical yellow-green of an expanded monolayer. This was an indication of some sort of irreversible aggregation, however a closer look made by depositing the monolayer on a TEM grid, as displayed in Figure 3.1 revealed it was in fact coagulation and sintering of the silver nanocubes into rounded, conjoined particles. This was a problem as the goal of the project was not only to study strongly coupled silver nanocubes, but to potentially study them at the quantum-tunneling limit. Populations of conjoined particles complicated this as observations of nanoparticles in conductive contact differ little from those in the quantum regime. ${ }^{38}$ Thus it was necessary to devise a way to prevent the coalescence of nanocube.
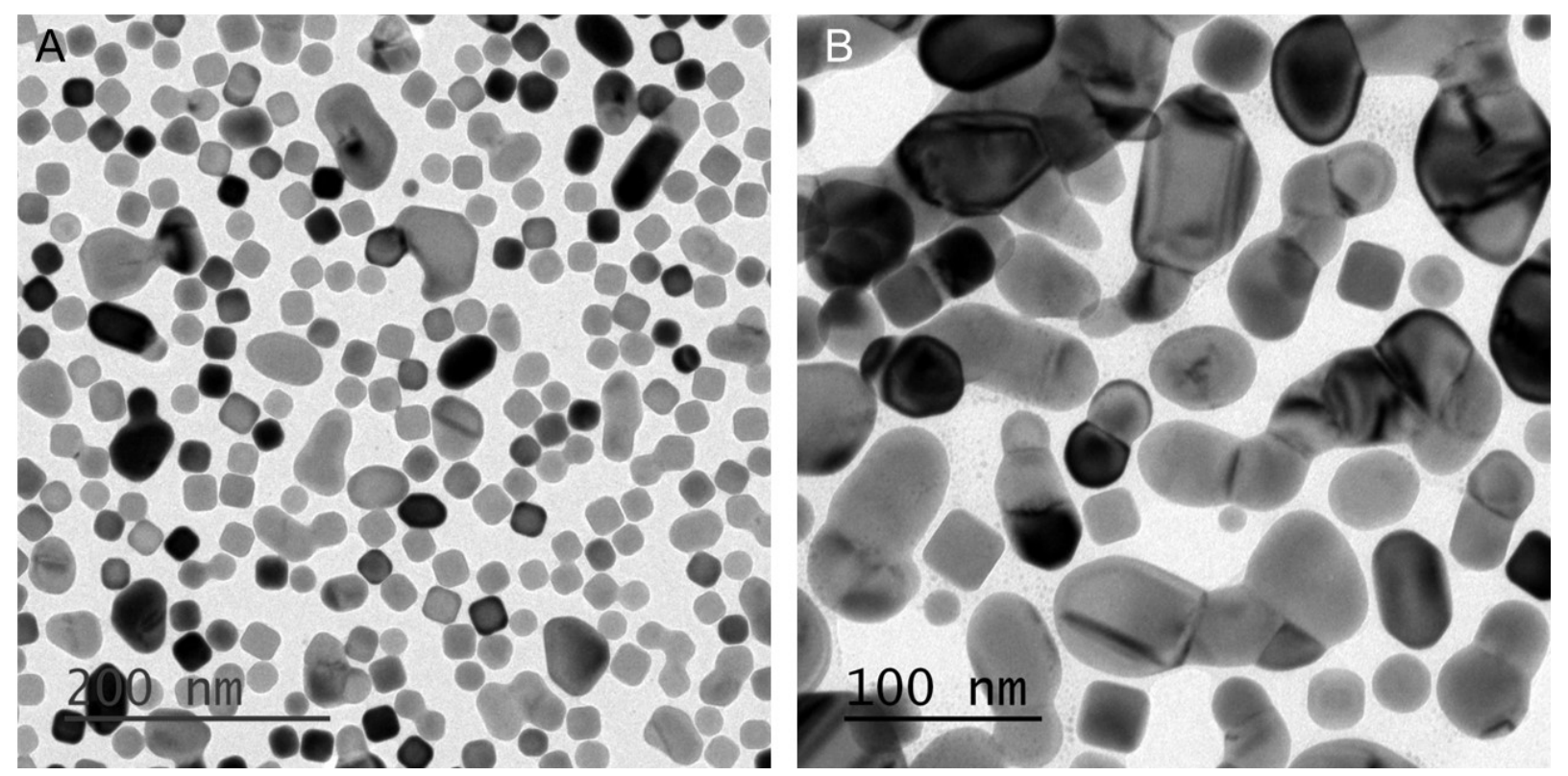

Figure 3.1 - Spontaneous coalescence of nanocube assemblies in Langmuir films, A) shows a moderate amount of coalescence and particle rounding, while B) gives a closer view of a heavily coalesced monolayer, though some cubes still remain intact.

To our knowledge there have been no previous reports of such coalescence of nanoparticles in Langmuir-Blodgett monolayers, so to understand why this is occurring one must 
consider what is unique about this system. First to consider, is that the LB monolayers where instances of this nanocube coalescence was noted involved the use of relatively small, as little as $25 \mathrm{~nm}$ in edge length. The corners and edges of these nanocubes, or other shaped nanoparticles, sustain very high surface energies for atoms located in these areas. ${ }^{137}$ When supplied with sufficient external energy, the atoms will alleviate said surface energy by diffusion to the face of the nanocube. ${ }^{138}$ This is why in actuality metal nanocubes are never a perfect cube, but are always contain some degree of truncation. Since these nanocubes are particularly small in comparison to those used in similar studies, ${ }^{93}$ the surface energies at the edges and corners are particularly high due to the low radius of curvature in these areas. As such, when at the air-water interface, in the presence of other nanocubes, the Ag surface atoms develop sufficient mobility for diffusion not only to their own faces, but to neighbouring nanocubes.

This sort of diffusion however is typically forbidden by steric repulsion caused by the surfactant or capping layer that colloidal nanoparticles posess. ${ }^{139}$ This sort of coalescence is typically only reported for the case of uncapped nanoparticles which have been immobilised onto a substrate. ${ }^{38,140}$ This brings us to the second condition which allows for the formation of these coagulated nanoparticle layers: the high lateral surface pressure exerted by the LangmuirBlodgett trough. These coalesced particles are typically only observed once the particles have been compressed to a high, solid phase surface pressures (in these cases typically greater than 6$7 \mathrm{mN} / \mathrm{m})$. We therefore hypothesise that these high surface pressures are sufficient in overcoming the steric hindrance of the capping agent and allow attractive van der Waals forces to bring the particles together and allow for diffusion of $\mathrm{Ag}$ atoms between nanoparticles. Note that in this case our capping agent is PVP, which is only loosely physisorbed to the surface, and with sufficient external force is easily displaceable. ${ }^{64,132}$ 
With this in mind, it is possible to prevent such catastrophic coagulation of nanocubes in the LB films, limiting the population of coalesced nanoparticles at high surface pressures. The two approaches used to limit the occurrence of this nanoparticle sintering were to: increase the average size of silver nanocubes used in future experiments, and to better control the amount of our passive phospholipid spacer, DOPC, in the LB monolayers. The presence of DOPC was found to have a modest effect at reducing aggregation at lower pressures, though high pressure monolayers could still become heavily coagulated as the lipid may be phase-separated from the nanoparticles at high pressure. The most effective means of preventing this aggregation was accomplished by using nanocube samples of larger average size. Figure 3.2 displays a monolayers of small (size) and slightly larger (size) nanocubes. While the smaller size nanocubes showed favourable extinction spectra and remained cubic when drop-casted, they became heavily truncated and rounded when dispersed at the air/water interface. While some instances of coalescence still occur in the larger nanocube sample, they are clearly more stable than the rounded sample.
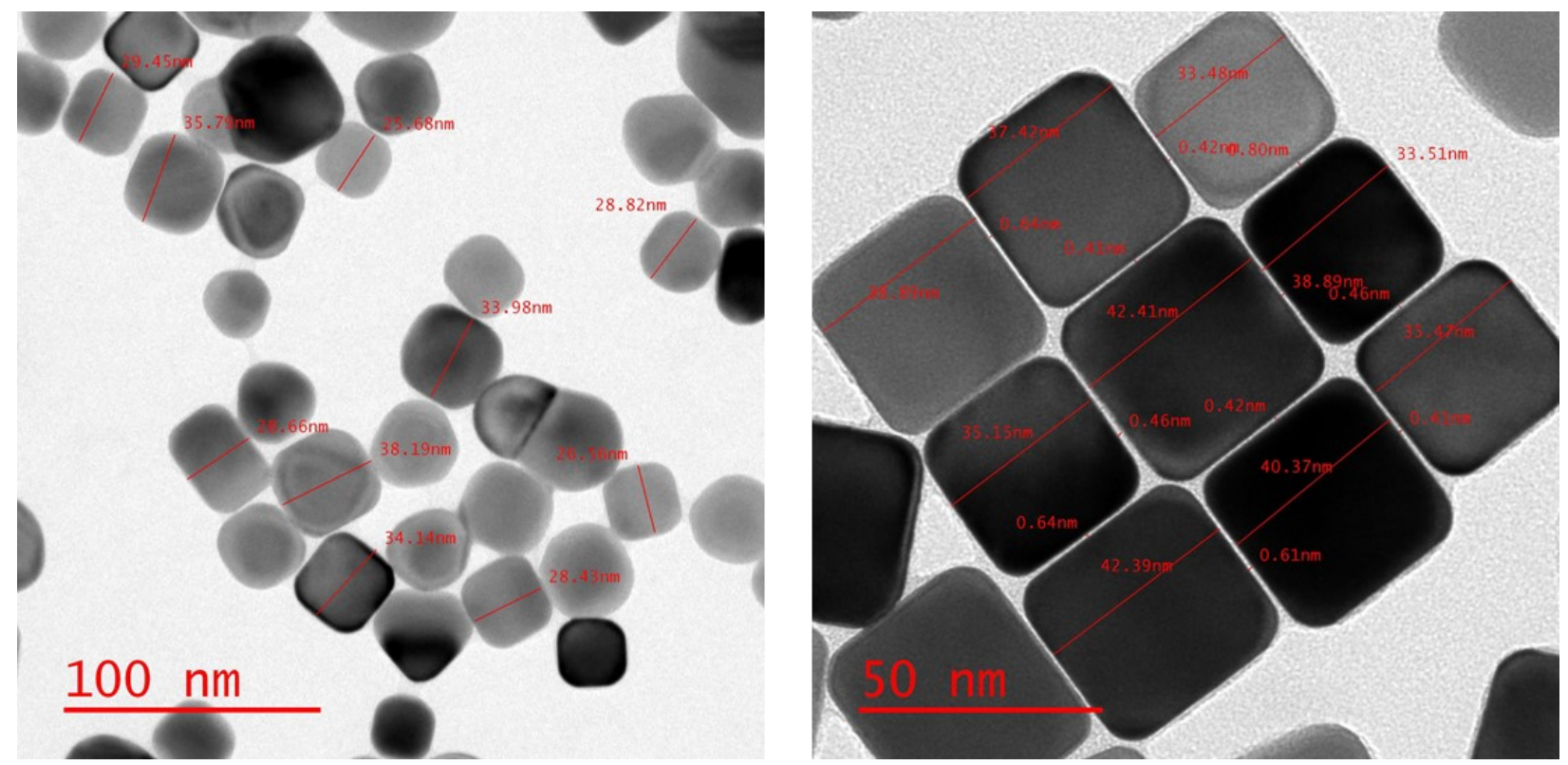

Figure 3.2 - The sample on the left with average cube size of $(29 \pm 4) \mathrm{nm}$ displays some coalescence of nanocubes while the sample on the right with cube size $(38 \pm 4) \mathrm{nm}$ is more stable to diffusion-driven agglomeration. 
Thus in future assemblies and syntheses, the aim was to produce slightly larger nanocubes. Sizes of about $40 \mathrm{~nm}$ were targeted, as this size was shown to increase stability of the nanocubes while maintaining the desired sharp and simple spectral features. Larger nanocubes will contain broad resonances and higher order multipolar modes by nature,${ }^{51,75}$ which may complicate the already broad features of the coupling modes in the nanoparticle assemblies.

\subsection{Surface Modification and Self-Assembly of Nanocubes by Alkanethiols}

While the Langmuir-Blodgett technique offers excellent control over nanoparticle density and yields assemblies with well-controlled optical properties, it does not offer precise control over the average interparticle distance. Due to this the coupling resonances that this study aims to explore are broad, and their precise position is not well controllable. To properly study coupled LSPRs in an ensemble, the generation of monolayers with monodisperse interparticle gaps would be ideal. An attractive route for this, which has in the past been frequently used for small Ag and Au nanoparticles would be self-assembly directed by alkanethiols. This method is capable of producing arrays of close-packed nanoparticles with controlled gap size, ${ }^{87}$ and is even compatible with the Langmuir-Blodgett technique. ${ }^{80}$ Given the merits of this sort of assembly, attempts were made to apply it to monolayers of silver nanocubes.

\subsubsection{Experimental}

\section{Surface modification of silver nanocubes by alkanethiols}

Materials: 1-Hexadecanethiol (C16, 95\%), 1-Dodecanethiol (C12, 98\%), and 1-Propanethiol (C3, 99\%) and Trimethoxy(propyl)silane were purchased from Sigma-Aldrich and used as is.

Silver nanocubes were synthesized with the same methods as described in Chapter 2. The nanocubes were re-dispersed in the solvent in which the ligand-exchange was to take place. 
Different solvent choices were experimented with, but the most successful exchanges were found to be in $\mathrm{ChCl}_{3}$ for hexadecanethiol and dodecanethiol, while ethanol or 1-propanol was used for the exchange of propanethiol. The nanocubes were dispersed in $10 \mathrm{~mL}$ of the selected solvent, and a volume of alkanethiol was added such that the final concentration in the vessel was $1 \mathrm{mM}$. The reaction vessel was sealed and stirred for $3 \mathrm{~h}-12 \mathrm{~h}$. The samples were precipitated and rinsed by centrifugation 3 - 5 times, redispersed in their original solvents and stored for later use.

Langmuir-Blodgett monolayers of the modified nanocubes were performed by redispersing them in $200 \mu \mathrm{L}$ of chloroform and then deposited onto the water surface drop-wise with a microsyringe. The monolayers were deposited at $15 \mathrm{mN} / \mathrm{m}$. The modified nanocubes were found not to adhere well to a typical glass slide, so slides were incubated for $1 \mathrm{~h}$ in $1 \%$ by volume trimethoxy(propyl)silane in methanol so as to make them hydrophobic. The monolayers were deposited by vertical lifting, monolayers were also deposited onto TEM grids.

\subsubsection{Results and Discussion}

An initial challenge was to prevent precipitation and aggregation of the nanocubes during the ligand-exchange reaction. This took some tuning as the overall polarity of the nanoparticles changes as the alkane length is changed. Hexadecanethiol (C16) modified cubes were non-polar, and only found to stable in chloroform, while on the other extreme propanethiol (C3) were relatively polar and easily dispersed in ethanol, but aggregated in chloroform. Even after identifying good solvent choices based on alkane chain length, another problem with this method was made apparent immediately upon attempting to purify the sample of excess alkane-thiols through centrifugation; irreversible aggregation of the nanocubes. After all attempts to resuspend the silver nanocubes after centrifugation and allowing for the heavy aggregates to decant, the natant nanocubes still showed some degree of aggregation, confirmed by the presence 
of an LSPR coupling mode in their extinction spectra. Attempts to make a LB monolayer of these particles are displayed in Figure 3.3. Despite the well-controlled interparticle distances, and well defined extinction spectra, this attempt failed to make a monolayer as multi-layer stacking is clearly evident in Figure 3.3 A and C.
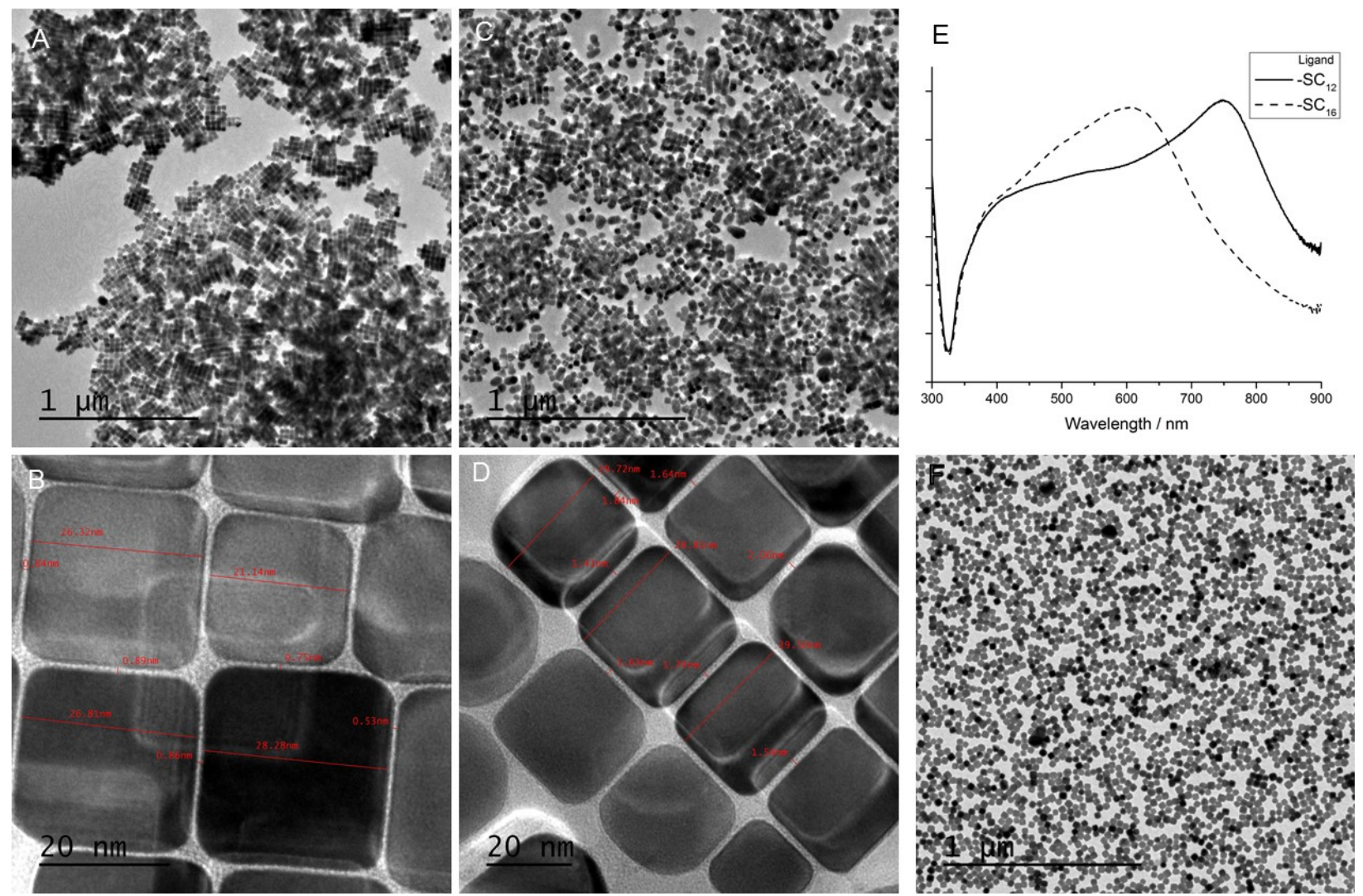

Figure 3.3 - Alkanethiol assembly of silver nanocubes, A) and B) Dodecanethiol (DDT, C12), gap size (0.8 \pm 0.1$) \mathrm{nm}$, C) and D) Hexadecanethiol (HDT, C16) assembly, gap size (1.7 \pm 0.2$) \mathrm{nm}$, E) Extinction spectra of DDT and HDT assemblies F) Propanethiol (PT, C3) assembly, does not aggregate, well dispersed at the air-water interface

Difficulties in alkanethiol substitution are not unheard of with large $\mathrm{Ag}$ or $\mathrm{Au}$ nanoparticles. Wei et al. noted that for particles larger than $10 \mathrm{~nm}$ tend to have planar facets which encourage close packed domains of alkanethiols with low chain mobility. ${ }^{139}$ Interdigitation of these chains leads to an overwhelmingly attractive van der Waals forces, resulting in a stable, minimal entropy system whereby little or no loss of entropy can be attained 
by the approach of another surface or solvent molecule. Thus large particles become locked in their position, with little that can be done to separate them.

An exception to this situation is observed in Figure $3.3 \mathrm{~F}$. This TEM image is of the monolayer produced after modification by propanethiol. While some aggregation was observed in the colloidal suspension, this sample was generally well-suspended in ethanol. It also appears to have been well-dispersed at the air-water interface. The polarizability of the nanoparticle itself allows particles modified with short-chain thiols $(<\mathrm{C} 4)$ to be suspended in polar solvents. ${ }^{80}$ In this instance they were sufficiently polar so as not to induce aggregation by solvophobic behaviour. This is unfortunate as the intended goal was potentially to produce assemblies of nanoparticles with angstrom-scale gaps in the attempt to observe quantumtunneling plasmonics in an ensemble of nanocubes.

While the above experiment did not succeed in its goal of producing a single-layer lattice of nanocubes with controlled interparticle distance, the lessons used here could be applied to this end. Such lattices of larger, faceted nanoparticles have been produced using similar ligands with significantly bulkier, multidentate head groups to give the alkane-chains greater mobility. ${ }^{139}$ Alternatively, selective face functionalization of nanocubes has been demonstrated, ${ }^{89}$ and may yield a route to producing dimers or small clusters of interacting nanocubes which can be studied for their distance dependence and observation of quantum-tunneling phenomena. While the use of solvophobic forces may not be a possible route to observing nanoparticles with gaps in the quantum regime, short dithiols provide a possible route into this sort of assembly. ${ }^{38}$ 


\section{$4 \quad$ Interacting Silver Nanocubes on High-Refractive Index Oxide Substrates}

The previous sections provided valuable insight into the optical properties of supported, strongly interacting silver nanocubes, and how to control their assembly. Much of the fundamental issues in the original study warranted further investigation. In this section we explore the near-field and far-field optical properties of strongly interacting silver nanocubes on high refractive index dielectrics. In particular, detailed quantification of the near-field effects as examined by SERS experiments are performed. Furthermore an attempt to spatially distinguish these near field effects is explored. This is done by using two Raman labels, DPPE-RhB as used previously is originally investigated in SERS experiments, but then is replaced by a selfassembled monolayer of benzenethiol. Benzenethiol is a popular Raman reporter in SERS experiments due to its strong ring breathing modes and it can be covalently attached to noble metal surfaces with ease. ${ }^{141}$

This work will explore the correlation between the far-field and near-field optical properties of the silver nanocube monolayers. Shifting the plasmon resonances of the silver nanocubes by changing the dielectric properties of the underlying substrate may be beneficial in improving the SERS enhancement of the nanocube assembly. Observed are cases when these interactions may be of benefit, but also where they may be a hindrance to SERS. 


\subsection{Experimental}

\subsubsection{Synthesis of Silver Nanocubes}

Chapter 3 demonstrated that large silver nanocubes were more stable than the smaller variety produced earlier. Growth of larger cubes using the method in Chapter 2 was difficult however, as an increasing population of right bipyramids, arising from singly twinned seeds, would be present in the sample with increasing reaction time. These were characterised by the appearance of a new LSPR resonance red of the dipolar mode of AgNC. Slightly larger cubes were also desired in order to shift the resonance into a desirable position, so as to line up the dipolar mode with the Raman excitation.

To facilitate the growth of slightly larger nanocubes, a chloride mediated process was used. This synthesis is otherwise identical to the previously stated method, with the following adjustment: the solution containing $0.8 \mathrm{mg} / \mathrm{mL}$ polyvinylpyrrolidone(PVP) also contained 7 $\mathrm{mg} / \mathrm{mL} \mathrm{NaCl}(120 \mathrm{mM})$. The addition of $\mathrm{NaCl}$ significantly slows the reaction rate due to competition between growth and the oxidative etching. Upon injection of silver the reaction turns yellow, quickly transitioning to an orange colour, before eventually turning brown, indicating the presence of small silver particles with a plasmon $\sim 400 \mathrm{~nm}$. No significant spectral changes occurred until about an hour into the reaction, after which the dipolar mode began red shifting steadily. The reaction was finally quenched after about 1 hour and 15 minutes when a distinct mode appeared at 350nm and the dipolar mode had reached $440-445 \mathrm{~nm}$.

Upon cooling, the reaction flask was diluted with ethanol, and the sample was centrifuged, redispersed in ethanol, and further centrifuged several times at $13000 \mathrm{~g}$ 's. After 5 rinses the sample is stored in ethanol where it remained stable. 


\subsubsection{Langmuir - Blodgett Monolayers}

Langmuir - Blodgett monolayers were fabricated as described in Chapter 2. However there were some slight modifications:

DOPC was still used in the cube/lipid ratio as discussed earlier, but the amount of Rhodamine B was adjusted such that the molar ratio of DPPE-RhB/DOPC added being varied between $1 \%-10 \%$.

For SERS experiments, monolayers of pure DPPE-RhB were produced. This was done by depositing $40 \mathrm{uL}$ of $0.56 \mathrm{mg} / \mathrm{mL}$ DPPE-RhB in chloroform onto the trough dropwise with a microsyringe. After allowing for chloroform evaporation, these monolayers were deposited onto all of the substrates that silver nanocube monolayers were deposited on for that set of experiments. Thus each silver nanocube monolayer had a DPPE-RhB reference of the same substrate to be used for SERS such that effects such as optical losses could be accounted for.

Substrates: Thin films of titanium oxide were fabricated by e-beam PVD from a $\mathrm{Ti}_{3} \mathrm{O}_{5}$ source. The film however is non-stoichiometric and will contain various oxidation states of $\mathrm{Ti}$, and thus will be referred to as $\mathrm{TiO}_{\mathrm{x}}$ or titanium oxide in general. In this set of experiments, slides of varying thickness of $\mathrm{TiO}_{\mathrm{x}}$ were cut into thin pieces $(\sim 5 \mathrm{~mm} \times 20 \mathrm{~mm})$ so several of them could be simultaneously deposited. This approach was chosen so as to keep the AgNC monolayers between different thicknesses of $\mathrm{TiO}_{\mathrm{x}}$ as identical as possible, which is facilitated by the excellent long range order in Langmuir-Blodgett monolayers. Simultaneous deposition is favourable to repeated depositions due to the disturbance provided by the vertical dipping of substrates, which make each layer less consistent. Prior to deposition the substrates were incubated in methanol for 20 minutes. 


\subsubsection{Post-modification of AgNC monolayers with benzenethiol (BT)}

Materials: Thiophenol (97\%) was acquired from Sigma-Aldrich and used as is. All solvents were de-oxygenated by purging with $\mathrm{N}_{2}$ due to the oxygen sensitivity of thiols.

Self-assembled monolayers (SAMs) of benzenethiol were deposited onto the silver nanocubes as an alternative label for SERS experiments. This was done after deposition onto the substrate as modification beforehand would alter the nanocube's behaviour at the air/water interface, making the properties of the monolayer more difficult to control. This was also done after SERS experiments involving DPPE-RhB as it is rinsed off in the process.

Before modification the substrates were rinsed with ethanol. Modification was done by immersing the substrates in $5 \mathrm{mM}$ benzenethiol in de-oxygenated ethanol in a sealed container where the atmosphere was purged by $\mathrm{N}_{2}$. The substrates were incubated for at least $2 \mathrm{~h}$. They were then removed from the BT solution and rinsed several times with ethanol to remove unbound BT. Samples were stored in an evacuated chamber due to the limited air stability of the SAMs after modification with thiols. ${ }^{142}$

\subsubsection{Characterization}

UV-Vis exctinction spectra of the colloidal nanocubes in ethanol and of deposited silver nanocube monolayers were recorded using a Shimadzu UV-2450 UV-Vis spectrophotometer. $\mathrm{UV}-\mathrm{V}$ is reflection and transmission spectra were collected on Ocean Optics fiber optic platform with a PX-2 pulsed xenon light source and USB-2000 UV-Vis detector. Transmission Electron Micrographs were collected on a FEI Tecnai G2 F20 TEM at the Carleton University Nanoimaging Facility. 


\subsubsection{Surface-Enhanced Raman Spectroscopy}

The instrument was modified from the one presented in chapter 2. The monochromator uses a different diffraction greating, and the setup is now coupled to an inverted microscope and micro-positioning stage.

Excitation was done using an $\mathrm{Ar} / \mathrm{Kr}$ ion laser (Coherent) at $458 \mathrm{~nm}, 488 \mathrm{~nm}, 514 \mathrm{~nm}$, $568 \mathrm{~nm}$, and $647 \mathrm{~nm}$, and was passed through an inverted microscope (Olympus IX-71) with a 20x objective and the SERS signal was collected through the same objective in a backscattering geometry. Raman spectra were collected using a single grating monochromator (Jobin Yvon, $640 \mathrm{~mm}$ focal length, 1200 lines $/ \mathrm{mm}$ ) with a liquid N2 cooled CCD camera (Princeton Instruments). The power on sample was measured to be between $2-5 \mathrm{~mW}$. SERS spectra were collected by WinSpec/32 software, processed in GRAMS/AI spectral data processing software, and cyclohexane was used for Raman shift calibration.

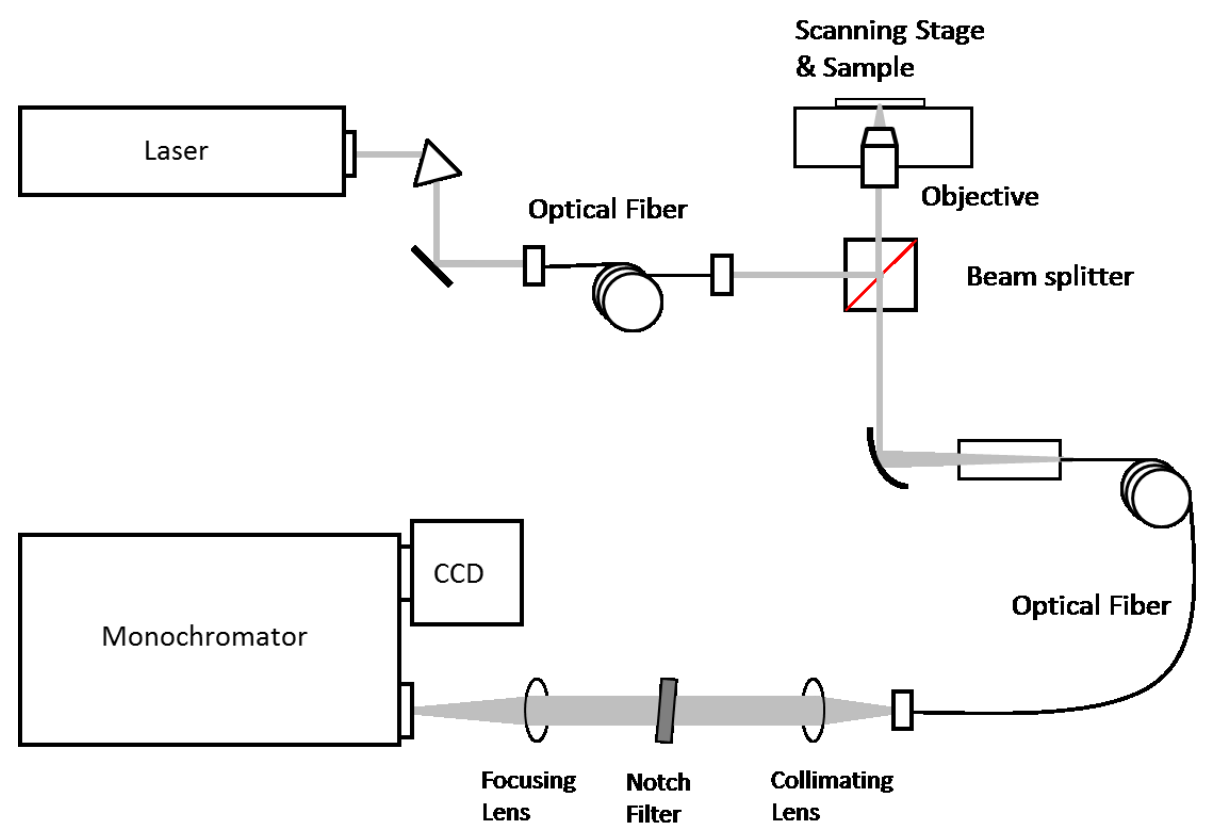

Figure 4.1 - Schematic diagram of the Raman Microscopy setup used in this set of experiments. The sample is palced on a translatable stage and excited in a backscattering geometry. This differs from the schematic in Figure 2.1 in that it is coupled into a microscope, yielding better spatial resolution, and signal/noise. The grating was exchanged from 1600 lines $/ \mathrm{mm}$ to 1200 lines $/ \mathrm{mm}$ to give a greater spectral range. 


\subsubsection{Calculation of SERS Enhancement Factor}

\section{Rhodamine-B}

The basic definition of the SERS enhancement factor is given by equation (1)

$$
\operatorname{SERSEF}(G)=\frac{I_{S E R S} / N_{\text {SERS }}}{I_{\text {Raman }} / N_{\text {Raman }}}
$$

In the case of our Rhodamine B measurements, both SERS and the reference monolayer are measured under identical conditions. As such the number of molecules $(N)$ can be replaced with the surface concentration of DPPE-RhB $(\Gamma)$ which can be elucidated from the LangmuirBlodgett isotherm.

$$
\operatorname{SERSEF}(G)=\frac{I_{S E R S} / \Gamma_{\text {SERS }}}{I_{\text {Raman }} / \Gamma_{\text {Raman }}}
$$

\section{Benzenethiol}

When using benzenethiol (BT) as a Raman probe, producing a reference monolayer is neither straightforward nor practical. As such the SERS enhancement factor must be determined using a liquid reference. It is therefore prudent to perform the necessary expansion of $N$ in equation (1) to consider the number of molecules being excited in both the case of the surface and liquid Raman samples:

$$
\operatorname{SERSEF}(G)=\frac{I_{S E R S} / \Gamma_{N C} \Gamma_{B T} A_{s, N C} A_{D}}{I_{\text {Raman }} / C_{B T} V_{e f f}}
$$

Where $\Gamma_{N C}$ and $\Gamma_{B T}$ are the surface concentrations of nanocubes and benzenethiol respectively, $A_{s, N C}$ is the average surface area of a silver nanocube, and $A_{D}$ is the area probed by the Raman instrument. $C_{B T}$ is the molecular concentration of the reference benzenethiol and $V_{\text {eff }}$ is the 
effective volume of the sample probed by the instrument. In this instance we use $V_{\text {eff }}$ can be determined in a number of ways, but is extremely subjective to experimental conditions and the instrument in use. ${ }^{110}$ To avoid errors associated with this value, the SERS EF is instead deduced using Raman cross sections, using DPPE-RhB, both in solution and as a monolayer, as an intermediate.

The SERS enhancement factor can also be determined by the ratio between Raman crosssections of a compound between a typical Raman measurement and SERS.

$$
\text { SERS EF }=\frac{\beta_{\text {SERS }}}{\beta_{\text {Raman }}}
$$

Therefore an alternative method would involve finding the Raman cross section of Benzene-thiol both in solution and on the surface. This can be done using equation (5) using a standard of known Raman cross section, in this case cyclohexane (CHex), under identical experimental conditions. Determination of the Raman cross section of BT in solution is straightforward under identical experimental conditions, assuming the difference in refractive index is negligible.

$$
\frac{I_{B T}}{I_{C H e x}}=\frac{\beta_{B T} C_{B T}}{\beta_{C H e x} C_{C H e x}}
$$

Rearrangement of equation (5) allows for the determination of the cross section of the benzenethiol mode under investigation, using the known cross section of cyclohexane again as the reference. ${ }^{143}$ To determine the SERS cross section of BT, Rhodamine B was used as an intermediate.

First the Raman cross section of Rhodamine B was determined in solution just as above in equation (5). Next we make the assumption that the Langmuir-Blodgett monolayers of 
Rhodamine B sit in a random orientation and the Raman cross section of Rhodamine B on this surface does not differ greatly from that in solution. By contrasting the SERS measurement with the RhB monolayer we can determine the SERS cross section of BT using equation (6).

$$
\frac{I_{S E R S, B T}}{I_{S, R h B}}=\frac{\beta_{S E R S, B T} \Gamma_{N C} \Gamma_{B T} A_{S, N C}}{\beta_{R h B} \Gamma_{R h B}}
$$

With the establishment of $\beta_{B T}$, and $\beta_{S E R S, B T}$, we can use equation (4) to estimate the SERS enhancement factor. For these calculations Raman measurements were made of neat cyclohexane, benzenethiol, and $10^{-4} \mathrm{M}$ DPPE-RhB in methanol. The surface concentration of benzenethiol, $\Gamma_{B T}$, was assumed to be 6.8 molecules $/ \mathrm{nm}^{2}$. This value, determined from closest packing based on the van der Waals radius of the molecule, ${ }^{144}$ is a standard value reported in most SERS literature. ${ }^{145}$ While in actuality the surface concentration is likely not this high (experimental evidence places the value in the range between 3.2 and 4.2 molecules $/ \mathrm{nm}^{2}$ ), ${ }^{146}$ this value will yield a conservative estimate of the enhancement factor. Due to the significant fluorescence background of $\mathrm{RhB}$ at resonant frequencies, but the significant weakness of Raman for the RhB at off-resonant excitation, the determination of the SERS EF in this manner was only possible at $488 \mathrm{~nm}$ excitation.

To apply this determination of the EF for all samples, a "relative enhancement" term $(G$ ) was devised:

$$
\text { Relative Enhancement }\left(G^{\prime}\right)=\frac{I_{S E R S} / \Gamma_{N C}}{I_{\text {Raman }}}
$$


The remainder of the terms in equation (3) that are set aside in equation (8) are constant throughout all samples and measurements (assuming the wavelength dependence of $V_{\text {eff }}$ is negligible) and can be considered constant, making this term proportional to the actual SERS enhancement factor:

$$
\begin{gathered}
\operatorname{SERSEF}(G)=\frac{I_{S E R S} / \Gamma_{N C}}{I_{\text {Raman }}} \times \frac{C_{B T} V_{e f f}}{\Gamma_{B T} A_{S, N C} A_{D}} \\
\operatorname{SERSEF}(G)=G^{\prime} \times K_{E F}
\end{gathered}
$$

To determine the SERS EF of BT at other excitation wavelengths, $K_{E F}$ from equation (9) was determined by applying the calculations in reverse for results at $488 \mathrm{~nm}$, and then the resultant constant was applied to the results at other excitation wavelengths. 


\subsection{Results and Discussion}

\subsubsection{Optical properties of interacting silver nanocubes on Titanium Oxide}

Silver nanocube monolayers were deposited on glass substrates with $\mathrm{TiO}_{\mathrm{x}}$ thin films with thicknesses of $0,5,10$, and $20 \mathrm{~nm}$. Figure 4.2 displays silver nanocube monolayers deposited at deposited at the liquid-condensed phase. At this pressure populations of both individual nanocubes and small groups and clusters exist, allowing for simultaneous observation of the

optical properties in both systems. For isolated nanoparticles, deposition on substrates of increasing refractive index produces behaviour as expected, ${ }^{18}$ with dipolar mode (D) red-shifting with increasing refractive index while the quadrupolar mode (Q) is relatively insensitive. Note that at $20 \mathrm{~nm} \mathrm{TiO}_{\mathrm{x}}$ the $\mathrm{D}$ mode becomes less sensitive to the refractive index of the substrate, no longer shifting linearly with increasing thin-film thickness. This behaviour has been observed previously, and is dependent on the size of the nanocube and the penetration depth of the electric field. ${ }^{51,147}$ The D-D mode displays a consistent red-shift with increasing refractive index of the substrate. Also noteworthy is the lack of saturation at $20 \mathrm{~nm} \mathrm{TiO}_{\mathrm{x}}$, indicating further penetration depth of the D-D plasmon into the substrate.

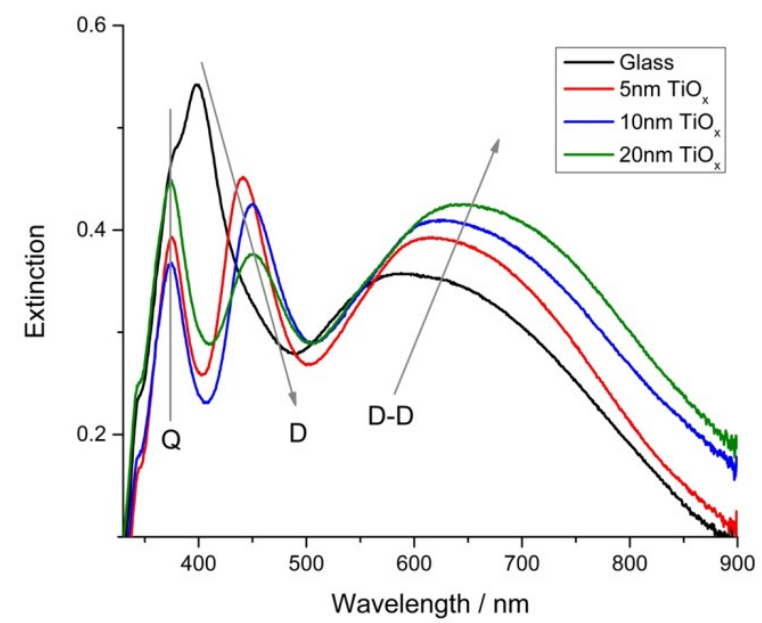

Figure 4.2 - UV/Visible extinction spectra for a monolayer deposited at $6 \mathrm{mN} / \mathrm{m}$ on varying thicknesses of $\mathrm{TiO}_{\mathrm{x}}$. Both the D and D-D modes red-shift with increasing thickness of $\mathrm{TiO}_{\mathrm{x}}$. While the D-mode shift becomes saturated at $20 \mathrm{~nm} \mathrm{TiO}_{\mathrm{x}}$ the D-D still continues to shift. 
As the deposition pressure of the monolayers increase we see a marked increase in extinction however the position of the D-D band remains relatively invariant. Thus by increasing the pressure we control the particle density of the substrate but exhibit little control over interparticle distances, but rather on the average size and population of clusters. Figure 4.3 displays the UV/Vis extinction spectra for monolayers deposited on both glass, and titania.
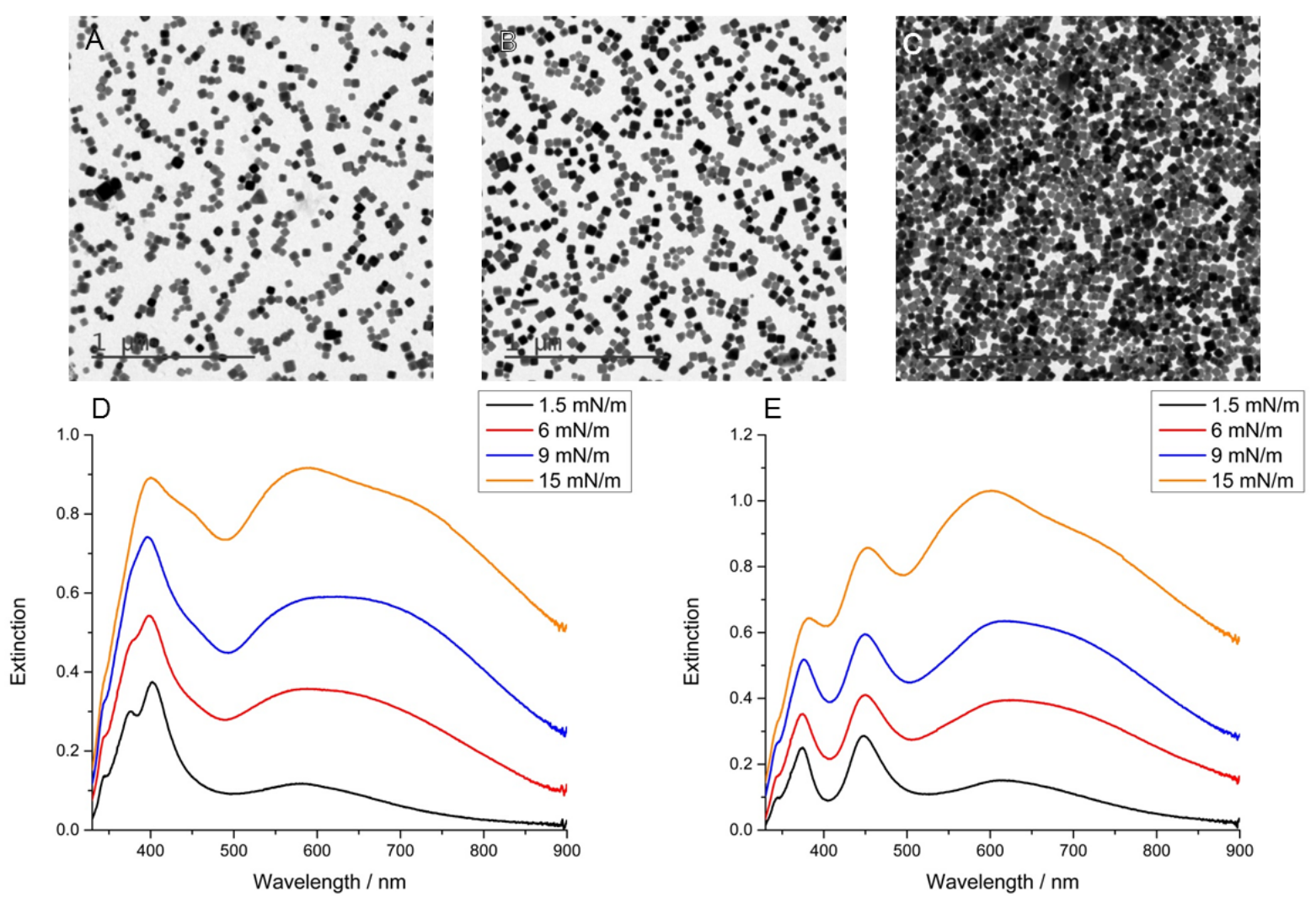

Figure 4.3 - A - C) TEM images of monolayers produced at $1.5,6$, and $15 \mathrm{mN} / \mathrm{m}$. D) UV-Vis spectra of aforementioned monolayers on Glass and $\mathrm{E}$ ) on a $10 \mathrm{~nm} \mathrm{TiO}_{\mathrm{x}}$ film. As the surface pressure increases the overall extinction of the monolayer increases, but there is little shift in the coupling modes. The $\mathrm{D}$ and $\mathrm{D}$-D modes on $\mathrm{TiO}_{\mathrm{x}}$ are more clearly distinguished, and red-shifted relative to their counterparts on glass.

\subsubsection{Surface-Enhanced Raman Spectroscopy}

Surface-Enhanced Raman Spectroscopy was once again used as a tool to probe the nearfield enhancements of the resultant silver nanocube monolayers. The deposited monolayers contained an amount of DPPE-RhB to be used as a label for SERS experiments. Since these molecules are incorporated into the Langmuir-Blodgett monolayer they lie at the surface of the 
substrate. In the second phase of the experiment this label is replaced by a SAM of benzenethiol on the surface of the nanocubes. The majority of the population of BT molecules will be located away from the substrate, thus by comparing the trends in these two labels we are able to spatially probe the location of field enhancements, examining the near-field properties of the monolayers at the bottom, and at the top of the cubes.
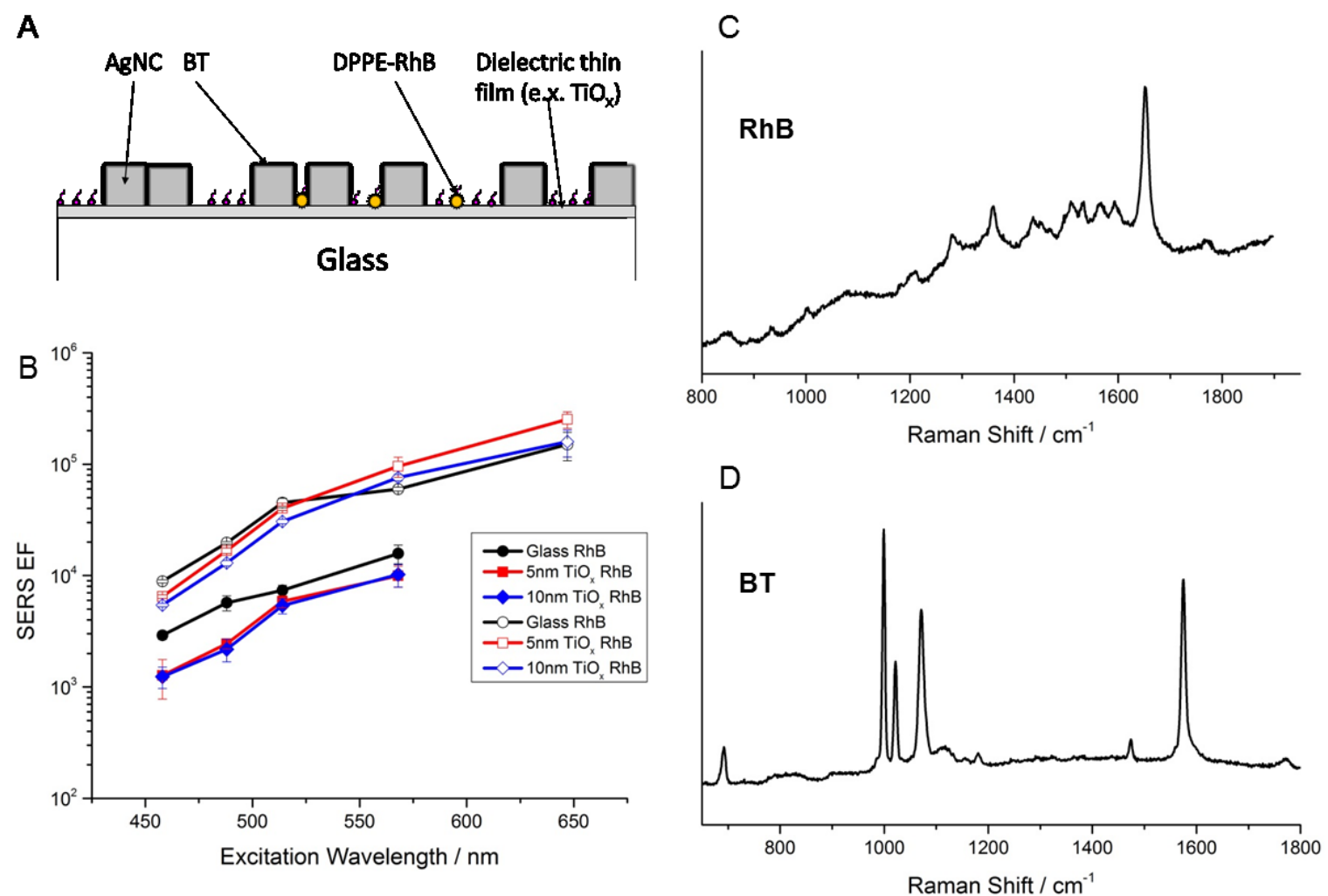

Figure $4.4-$ A) Schematic showing the design of the monolayer. DPPE-RhB is at the surface of the monolayer while modification by BT is selective to the AgNC surface. B) SERS Enhancement Factor as a function of wavelength for both Raman reporters; DPPE-RhB and Benzenethiol (BT). Higher refractive index substrates show less field enhancement at the surface, while this trend is not maintained with $\mathrm{BT}$ as the label. At longer wavelengths $\mathrm{TiO}_{\mathrm{x}}$ is shown to improve SERS against glass with BT as the label located away from the substrate. C) and D) SERS spectra collected at $488 \mathrm{~nm}$ are displayed for both RhB and BT.

The SERS enhancements factors recorded on each substrate at different excitation wavelengths are displayed in Figure 4.5, overlaid with the extinction spectra of monolayers used in this experiment. Refer also to Figure 4.4 in which this trend is more clearly displayed. In all cases the highest enhancement is recorded on glass, and subsequently decreases with increasing 
thickness of the titania thin films. The most pronounced difference is when the excitation is at $458 \mathrm{~nm}$, which is in resonance with the dipolar mode for nanocubes on titanium oxide. It was originally hypothesized that by shifting the resonance to coincide with the energy of the excitation beam the enhancement could be increased. Instead the opposite effect is observed, but this could be explained by the spatial separation between the D and Q mode induced by the interaction with a dielectric substrate. ${ }^{18}$ As the refractive index of the substrate increases the electric field component of the D mode is expected to be located deeper within the substrate. As a result molecules at the surface will feel less electric field enhancement.

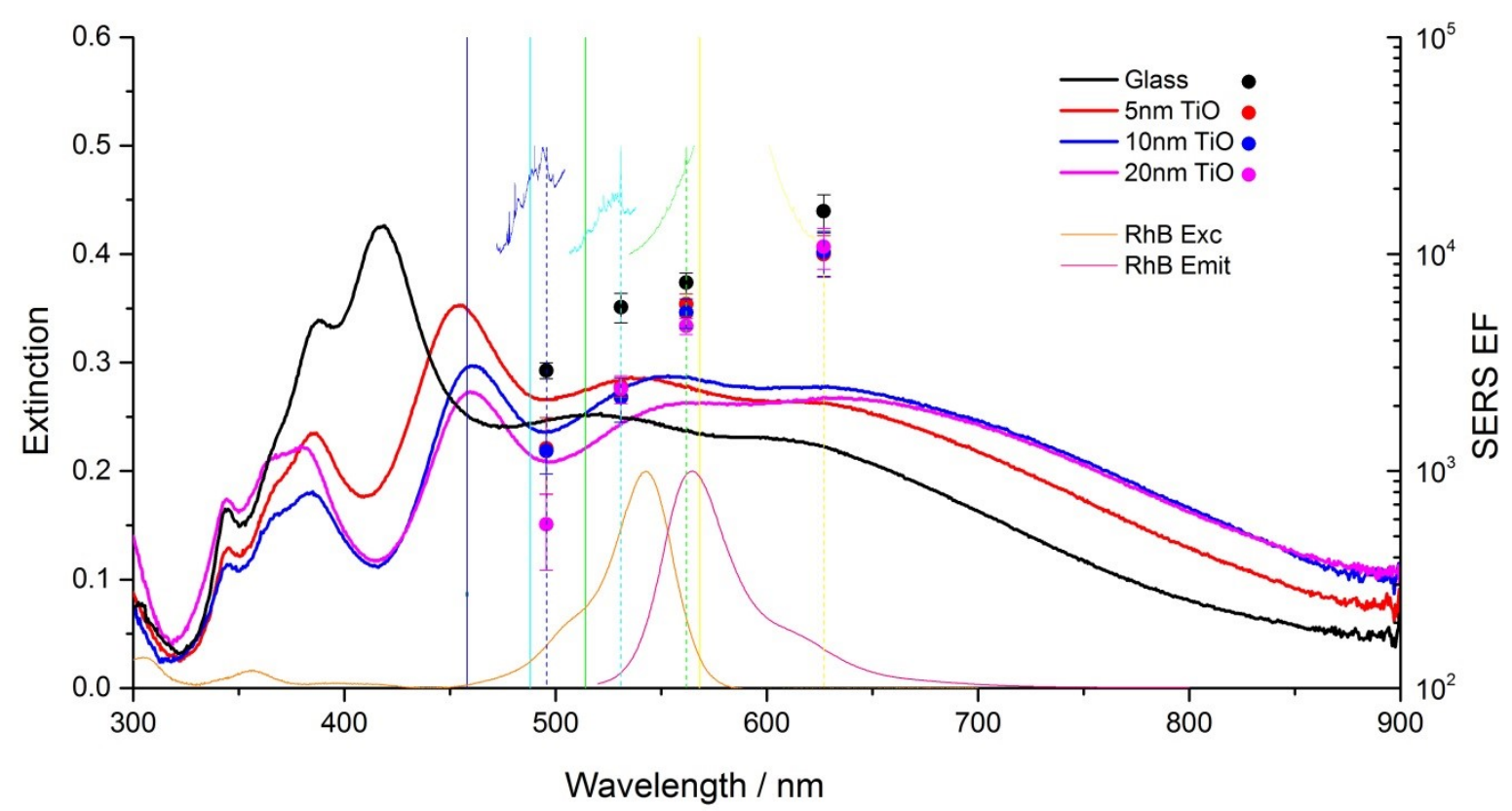

Figure $4.5-\mathrm{UV} /$ Vis extinction spectra of the monolayers deposited at $1.5 \mathrm{mN} / \mathrm{m}$, overlayed with DPPE-RhB SERS Enhancement Factors (points), excitation lines $(458 \mathrm{~nm}, 488 \mathrm{~nm}, 514 \mathrm{~nm}, 568 \mathrm{~nm})$, and a sample Raman spectrum collected at each excitation wavelength. In general, the enhancement factors increase as the wavelength becomes longer. SERS EF on glass (black points) are always the highest, despite the improved resonance conditions with the excitation source at $458 \mathrm{~nm}$. Excitation and emission spectra of $\mathrm{RhB}$ are also overlayed.

As the excitation wavelength is moved into the red part of the spectrum, the enhancement factor increases significantly. This is the logical result of excitation of the coupled plasmonic modes, which will produce exceptionally large enhancement in the junction between 
nanoparticles. The disparity between enhancement factors also becomes narrower as the coupling modes are probed. This indicates the hybridized coupling modes are less spatially separated than their non-interacting counterparts.

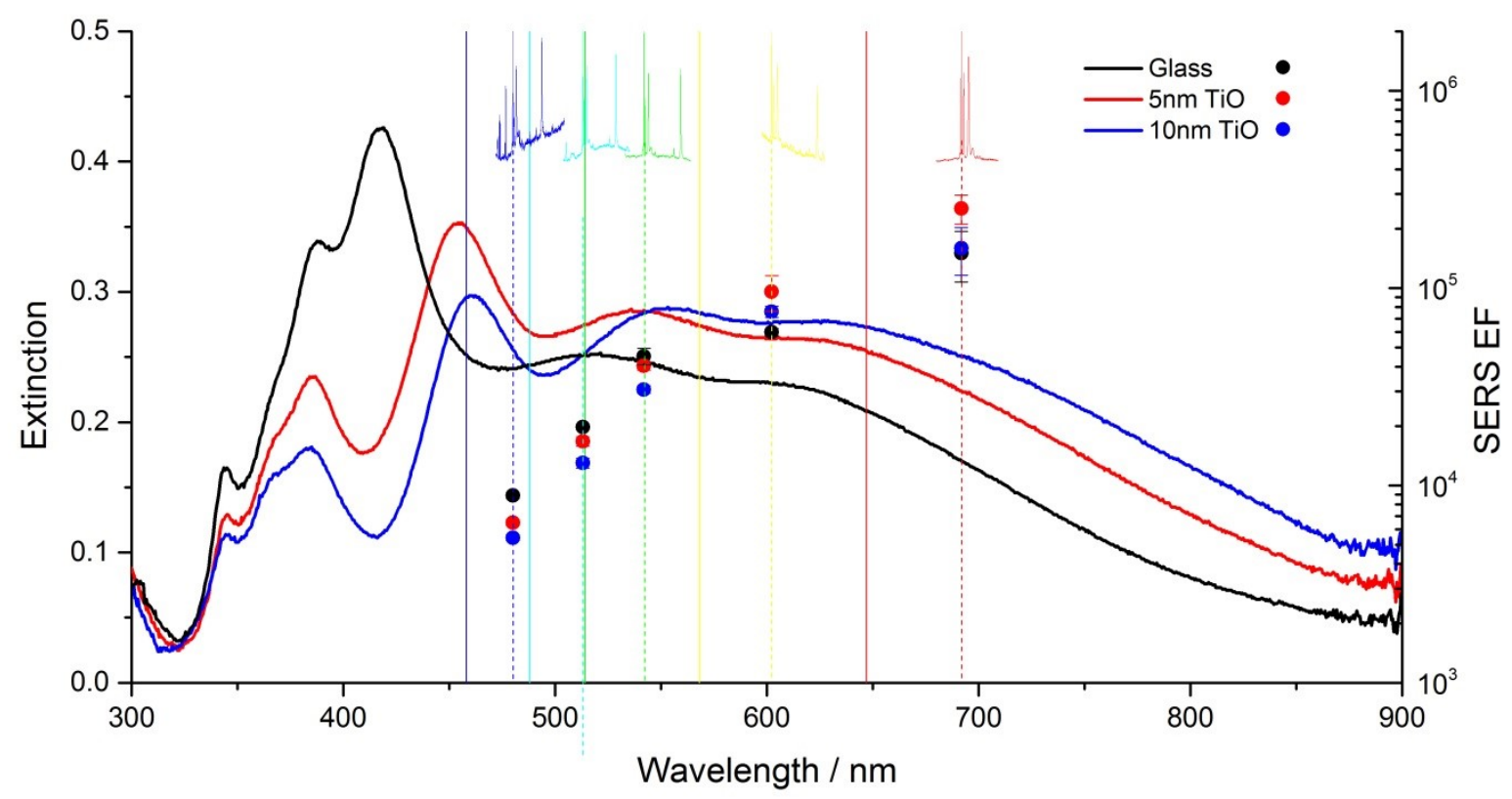

Figure 4.6 - UV/Vis extinction spectra of the monolayers deposited at $1.5 \mathrm{mN} / \mathrm{m}$, overlayed with BT SERS Enhancement Factors (points), excitation lines (458nm, 488nm, 514nm, 568nm, 647nm) and a sample Raman spectrum collected at each excitation wavelength. Again the enhancement factors increase as the excitation is longer. At longer wavelengths glass (black dot) does not display the highest enhancement factor, but rather improved SERS is observed on $\mathrm{TiO}_{\mathrm{x}}$.

Benzenethiol was used as an alternate label for SERS experiments, the wavelengthdependent results are summarized in Figure 4.4 and Figure 4.6. BT provides the additional advantages of not being reliant on resonant effects, and being selective to the surface of the nanocubes. SERS for this probe yielded a strong Raman scattering signal across the entire spectrum of excitation wavelengths. As observed with SERS of DPPE-RhB, the enhancement factor increases as the excitation wavelength is moved to lower energies. However the disparity between the observed enhancements across different substrates is significantly lower than in the case of DPPE-RhB. In fact the SERS EF on titania thin films begin to surpass those recorded on 
glass when $\lambda_{\text {exc }} \geq 568 \mathrm{~nm}$. What is displayed is decreased sensitivity to the substrate on the nearfield enhancement properties away from the substrate.
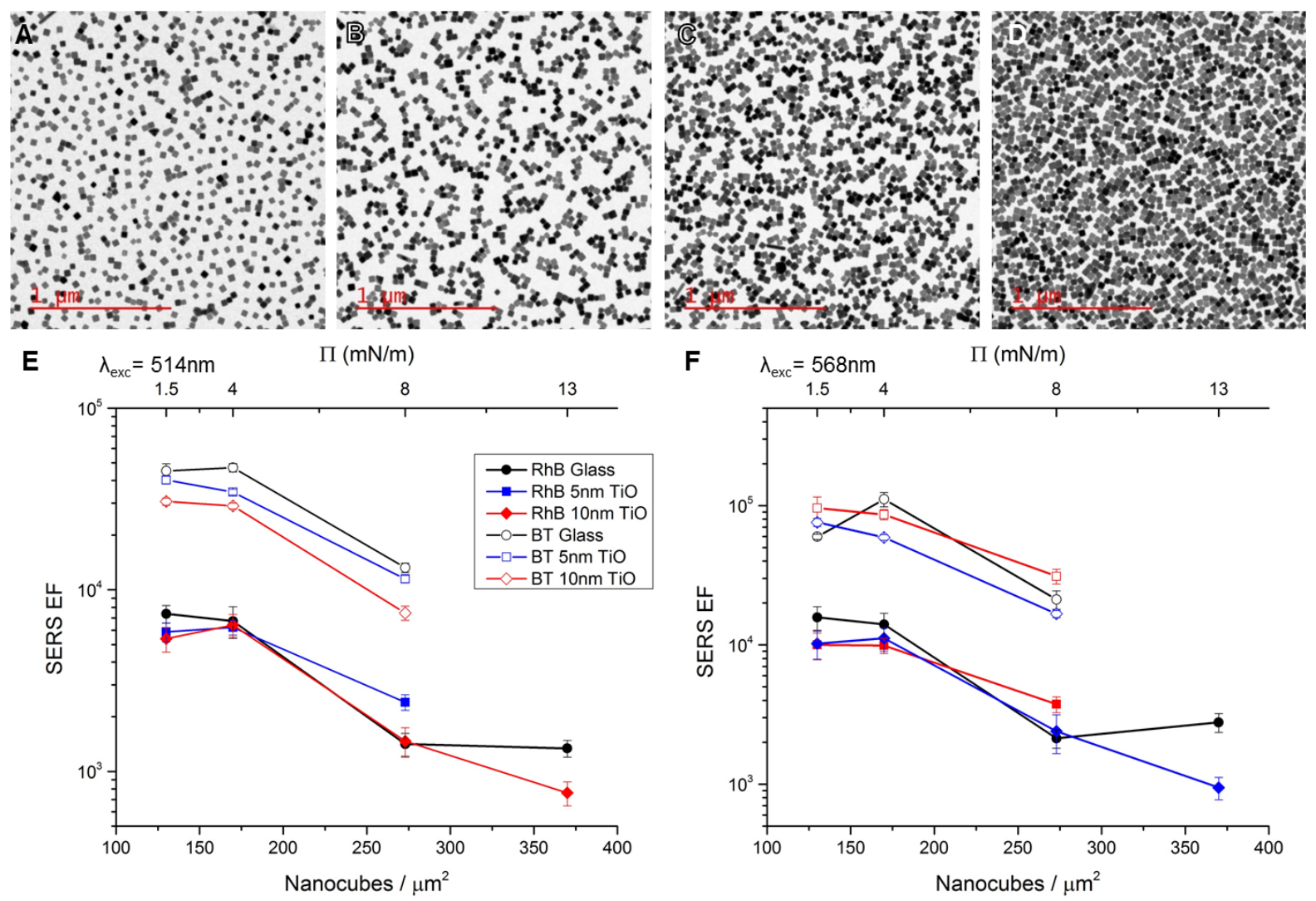

Figure 4.7 - A - D) TEM images of monolayers produced at $1.5 \mathrm{mN} / \mathrm{m}, 4 \mathrm{mN} / \mathrm{m}, 8 \mathrm{mN} / \mathrm{m}$, and $13 \mathrm{mN} / \mathrm{m}$. E) and F) SERS EF as a function of particle density for $514 \mathrm{~nm}$ and $568 \mathrm{~nm}$ excitation wavelengths. The lowest pressure monolayers display the highest enhancement factor

The enhancement factors plotted against particle density are displayed in Figure 4.7. The SERS EF decreases with increasing particle density. It has been shown that as plasmonic modes mix with a greater population of neighbours the maximum E-field enhancement in the junction decreases, ${ }^{148}$ and the presence of this effect is invoked here. A sudden decrease, at least half an order in magnitude, in enhancement factor between nanocubes deposited at the liquid-expanded and liquid-condensed phases. It should also be noted that there is a distinction between the absolute intensities of the Raman signals and the enhancement factor. The enhancement factor scales negatively much more rapidly in the dense monolayers in comparison to the intensity due 
to the fact that the number of reporter molecules being observed increases with the monolayer's pressure. This effect is particularly amplified with the use of DPPE-RhB as it 1) may be contained anywhere in the monolayer, thus a large portion of the molecules may not experience enhancement at all, and 2) at high pressures phase separation between the lipid and nanoparticle components can occur. The latter will result in a large fraction of the molecules experiencing very little of the enhanced electric field. In fact part of the reason DOPC is used in the monolayers is to reduce this phase separation, as it acts as a pseudo-solvent for DPPE at the air/water interface. It should also be noted that, the interparticle distance between nearly touching cubes, particularly in the high density monolayers, was in the sub-nanometer range, as observed by TEM. Since DPPE-RhB is a considerably bulky molecule, it is unlikely to exist in these junctions where the hotspots are likely to reside.

Likewise, with junctions this small, it cannot be certain the surfaces in the junction could have allowed the formation of a SAM of BT. In fact in some areas the average interparticle distance was observed to average $\sim 0.5 \mathrm{~nm}$, which in all likelihood is too small of an area to be filled by BT. Furthermore, DDA calculations by El-Sayed et al. have demonstrated that when the interparticle distance is $<2 \mathrm{~nm}$ the most intense hot spot is formed between the faces of the nanocubes, as opposed to the edges and corners when the spacing is $>4 \mathrm{~nm}{ }^{149}$ The improbability than many BT molecules, if any, reside in the hotpsot would explain the relatively weak SERSenhancement factors observed in comparison to that expected of strongly interacting cubes. ${ }^{150}$ The onset of quantum-tunneling and charge transfer effects in such small gap sizes may also be contributing factor to the weak enhancement. ${ }^{151}$ 


\subsubsection{Correlation between Near-field Enhancements with the Far-field Optics}

One of the goals of this experiment was to determine if the control over plasmon resonance position we attain by tuning the dielectric properties of the substrate can be used to maximize SERS enhancement by moving the resonances to coincide with the excitation beam. The clearest use of this is at $458 \mathrm{~nm}$, where the D mode can be shifted to nearly perfectly coincide with this laser source. However as previously discussed, excitation of this mode does not generate the most significant hotspots that occur between coupled nanocrystals. Also the spatial separation induced by plasmon hybridization in fact hinders the enhancement of this mode.

As the excitation laser is moved to longer wavelengths we begin to excite the coupling resonances, increasing the observed enhancement. While at the surface glass consistently remains the best substrate choice according to the DPPE-RhB results, we can see with the BT probe that at excitation wavelengths $\geq 568 \mathrm{~nm}$, the excitation better coincides with the red-shifted resonances on the titania substrate. Note, however that the enhancement factors do not follow the extinction spectra explicitly. The highest enhancement factors are recorded at $647 \mathrm{~nm}$, where there the extinction of the coupled plasmons begin to decrease. Closer investigations into the far-field optical properties were also made by transmission and reflection spectroscopy. Figure 4.8 displays these spectra on titania, in which the loss of transmission that did not correspond to reflection was assumed to be due to absorption. Again no simple, clear trend between the farfield optics and near-field enhancement can be made. Indeed it has been noted in other studies that in plasmonic nanostructures with a significant amount of small gaps, there is a breakdown in

any simple relationship between the optical properties and the observed SERS EF. ${ }^{152}$ This is because the SERS EF is inherently dependent only on resonances which produce a hotspot, while 
the far-field contributions will be the result of any number of interacting and individual modes, which is further complicated in nanoparticle ensembles. ${ }^{122}$ Additionally, contribution from dark modes, not normally observable in the far-field optical spectra, have been shown to be capable of providing a significant contribution to SERS enhancement. ${ }^{153}$
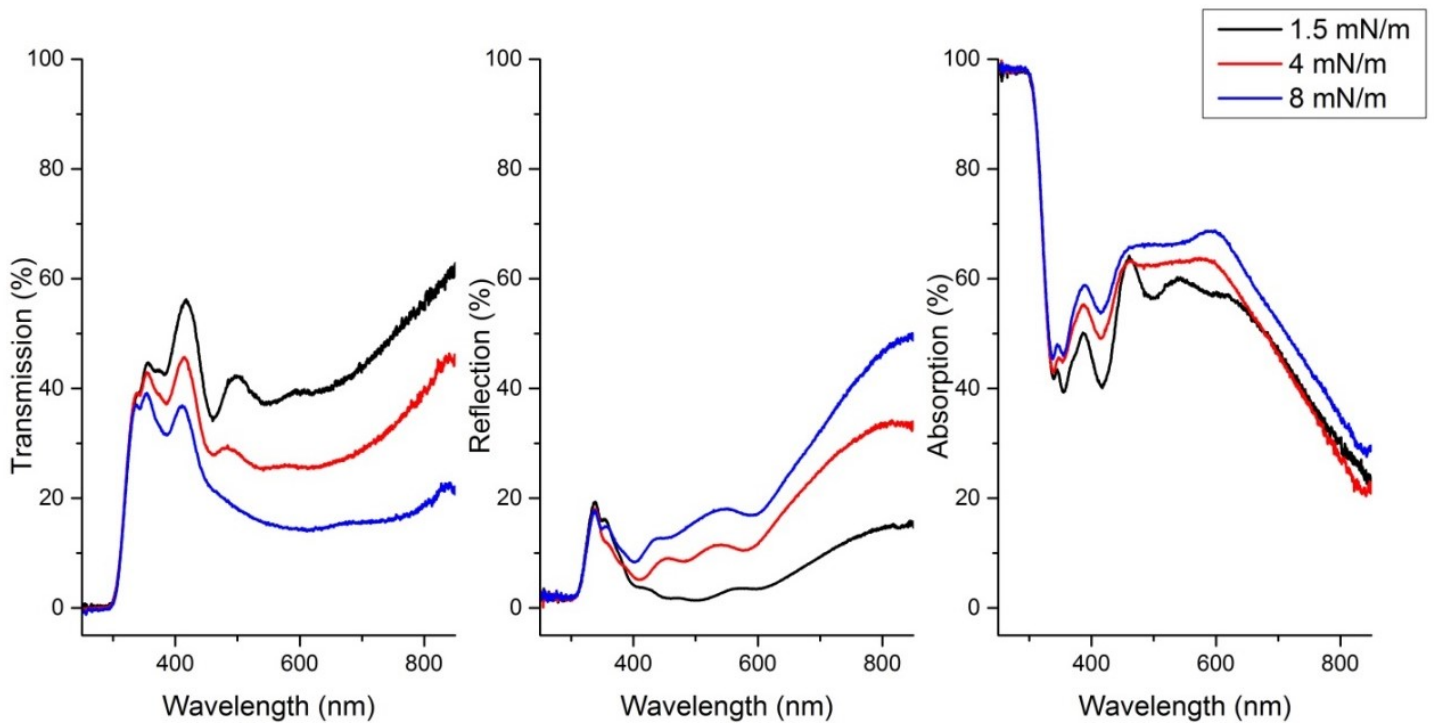

Figure 4.8 - Transmission, reflection, and absorption spectra of silver nanocube monolayers on $10 \mathrm{~nm} \mathrm{TiO}_{\mathrm{x}}$. Light that is neither transmitted nor reflected is assumed to be absorbed. With increasing particle density the films become more reflective, however the absorption only moderately increases. None of these optical properties clearly coincide with SERS EF trends observed in Figure 4.4.

\subsection{Conclusions}

The ability to red-shift coupling modes by increasing the refractive index of the substrate is demonstrated. As the refractive index of the substrate increases, the E-field near the substrate is diminished as it is drawn deeper into the substrate. This results in reduced SERS intensity of the silver nanocube monolayers near the surface, even when the plasmon modes are in better resonance with the excitation light. The ability to improve E-field enhancement away from the substrate by increasing the refractive index is demonstrated. This improvement does not clearly coincide with the far-field optical properties of the monolayer. The spatial separation of nearfield enhancement is experimentally demonstrated and may be applied towards the optimization of SERS substrates. 


\section{Concluding Remarks}

Presented in this work is the ability to tune the plasmon resonances in ensembles of interacting nanocubes by interactions with eachother and with the underlying substrate. The near-field spatial separation of certain plasmonic modes was experimentally demonstrated through SERS. Electric-field enhancements in the dipolar mode are on average reduced with increasing refractive index of the substrate. Electric fields near the surface of assemblies are reduced across the board with increasing refractive index of the substrate. It was demonstrated however, that high refractive index substrates can improve SERS signals for probes located away from the surface of the underlying substrate. Some correlation between the near-field and farfield optical properties of the nanocube assemblies is demonstrated, however correlation between the near-field and far-field properties is also proven to be complicated, and no simple relationship can be drawn across the entire spectrum. This work has presented an in-depth analysis of nanoparticle interactions, and how nanoparticle-substrate choices can be applied towards the optimization of surface enhanced Raman spectroscopy.

In Chapter 2 the presence of charge-transfer phenomena are believed to be responsible for the unique optical properties and reduced field enhancement observed in these silver nanocube assemblies. Such charge transfer events, such as in quantum tunneling of neartouching nanocrystals, can also be responsible for the reduced field enhancement observed in tightly packed nanocube assemblies. Even without the presence of these effects the average field enhancement in large-range aggregates is believed to be reduced in comparison to small clusters and dimers. As such the ideal geometry for SERS substrates utilizing silver nanocubes is a 
consistent assembly with long-range order that includes a high population of such small clusters, as opposed to a continuous array of strongly interacting particles.

Future work in this area would thus focus on the controlled assembly of silver nanocubes in such an array to maximize the average SERS enhancement. Additionally such assembly strategies could also be used for detailed study of quantum tunneling, substrate mediated charge transfer, and other unique plasmonic effects and interactions. Indeed the origins of the optical properties demonstrated in Chapter 2 are not fully understood. Future work could entail the indepth study of silver nanocrystals ensembles on a silicon surface with control over oxide thickness, dopants, and free-carrier density in order to study the unique plasmonic charge transfer effects that were observed. Future work could also focus on producing unique nanoparticle assemblies, such as those in the quantum tunneling regime, or unique hybrid particles. The ability to control the shape and assembly of noble metal nanocrystals has advanced considerably in recent years to the point where almost any conceivable nanoscale assembly should not be considered out of the reach of possibility. 


\subsection{Statement of Contributions}

Items included in this work have been featured in:

Ahamad, N.; Prezgot, D.; Ianoul, A. Patterning Silver Nanocubes in Monolayers Using Phase Separated Lipids as Templates. J. Nanoparticle Res. 2012, 14, 724.

Bottomley, Adam, Daniel Prezgot, and Anatoli Ianoul. Plasmonic properties of silver nanocube monolayers on high refractive index substrates. Applied Physics A 2012, 109.4, 869-872.

Bottomley, A.; Prezgot, D.; Staff, A.; Ianoul, A. Fine Tuning of Plasmonic Properties of Monolayers of Weakly Interacting Silver Nanocubes on Thin Silicon Films. Nanoscale 2012, 4, 6374-6382.

Prezgot, D.; Ianoul, A. Optical Properties of Strongly Interacting Supported Silver Nanocube Monolayers. Proc. SPIE 2013, 8597, 85970A.

Prezgot, D.; Ianoul, A. Substrate-Induced Effects on the Plasmonic Properties of Strongly Coupled Silver Nanocubes. Proc. SPIE 2014, 8996, 899612. 


\section{Bibliography}

(1) Barnes, W. L.; Dereux, A.; Ebbesen, T. W. Subwavelength Optics. Nature 2003, 424, 824-830.

(2) Mayer, K. M.; Hafner, J. H. Localized Surface Plasmon Resonance Sensors. Chem. Rev. 2011, 111, 3828-3857.

(3) Willets, K. a; Van Duyne, R. P. Localized Surface Plasmon Resonance Spectroscopy and Sensing. Annu. Rev. Phys. Chem. 2007, 58, 267-297.

(4) Zeman, E. J.; Schatz, G. C. An Accurate Electromagnetic Theory Study of Surface Enhancement Factors for Ag, Au, Cu, Li, Na, AI, Ga, In, Zn, and Cd. J. Phys. Chem. C 1987, 91, 634-643.

(5) Blaber, M. G.; Arnold, M. D.; Ford, M. J. A Review of the Optical Properties of Alloys and Intermetallics for Plasmonics. J. Phys. Condens. Matter 2010, 22, 143201.

(6) Khlebtsov, N. G.; Dykman, L. a. Optical Properties and Biomedical Applications of Plasmonic Nanoparticles. J. Quant. Spectrosc. Radiat. Transf. 2010, 111, 1-35.

(7) Jensen, T. R.; Duval, M. L.; Kelly, K. L.; Lazarides, A. a.; Schatz, G. C.; Van Duyne, R. P. Nanosphere Lithography: Effect of the External Dielectric Medium on the Surface Plasmon Resonance Spectrum of a Periodic Array of Silver Nanoparticles. J. Phys. Chem. B 1999, 103, 9846-9853.

(8) Sherry, L. J.; Chang, S.-H.; Schatz, G. C.; Van Duyne, R. P.; Wiley, B. J.; Xia, Y. Localized Surface Plasmon Resonance Spectroscopy of Single Silver Nanocubes. Nano Lett. 2005, 5, 2034-2038.

(9) Kosuda, K. M.; Bingham, J. M.; Wustholz, K. L.; Van Duyne, R. P. Nanostructures and Surface-Enhanced Raman Spectroscopy. In Comprehensive Nanoscience and Technology; Oxford: Academic Press, 2011; Vol. 3, pp. 263-301.

(10) Rycenga, M.; Cobley, C. M.; Zeng, J.; Li, W.; Moran, C. H.; Zhang, Q.; Qin, D.; Xia, Y. Controlling the Synthesis and Assembly of Silver Nanostructures for Plasmonic Applications. Chem. Rev. 2011, 111, 3669-3712.

(11) Xia, Y.; Xiong, Y.; Lim, B.; Skrabalak, S. E. Shape-Controlled Synthesis of Metal Nanocrystals: Simple Chemistry Meets Complex Physics? Angew. Chemie Int. Ed. 2009, $48,60-103$.

(12) Noguez, C. Surface Plasmons on Metal Nanoparticles: The Influence of Shape and Physical Environment. J. Phys. Chem. C 2007, 111, 3806-3819. 
(13) Meier, M.; Wokaun, A. Enhanced Fields on Large Metal Particles: Dynamic Depolarization. Opt. Lett. 1983, 8, 581-583.

(14) Kelly, K.; Coronado, E. The Optical Properties of Metal Nanoparticles: The Influence of Size, Shape, and Dielectric Environment. J. Phys. Chem. B 2003, 107, 668-677.

(15) Evanoff, D.; Chumanov, G. Size-Controlled Synthesis of Nanoparticles. 1. "Silver-Only" Aqueous Suspensions via Hydrogen Reduction. J. Phys. Chem. B 2004, 108, 1394813956.

(16) Gómez, D. E.; Teo, Z. Q.; Altissimo, M.; Davis, T. J.; Earl, S.; Roberts, a. The Dark Side of Plasmonics. Nano Lett. 2013, 13, 3722-3728.

(17) Schertz, F.; Schmelzeisen, M.; Mohammadi, R.; Kreiter, M.; Elmers, H.-J.; Schönhense, G. Near Field of Strongly Coupled Plasmons: Uncovering Dark Modes. Nano Lett. 2012, $12,1885-1890$.

(18) Zhang, S.; Bao, K.; Halas, N. J.; Xu, H.; Nordlander, P. Substrate-Induced Fano Resonances of a Plasmonic Nanocube: A Route to Increased-Sensitivity Localized Surface Plasmon Resonance Sensors Revealed. Nano Lett. 2011, 11, 1657-1663.

(19) Lee, Y. H.; Chen, H.; Xu, Q.-H.; Wang, J. Refractive Index Sensitivities of Noble Metal Nanocrystals: The Effects of Multipolar Plasmon Resonances and the Metal Type. $J$. Phys. Chem. C 2011, 115, 7997-8004.

(20) Wei, H.; Xu, H. Hot Spots in Different Metal Nanostructures for Plasmon-Enhanced Raman Spectroscopy. Nanoscale 2013, 5, 10794-10805.

(21) Deeb, C.; Zhou, X.; Miller, R.; Gray, S. K.; Marguet, S.; Wiederrecht, G. P.; Bachelot, R. Mapping the Electromagnetic Near-Field Enhancements of Gold Nanocubes. J. Phys. Chem. C 2012, 116, 24734-24740.

(22) Kim, D.-S.; Heo, J.; Ahn, S.-H.; Han, S. W.; Yun, W. S.; Kim, Z. H. Real-Space Mapping of the Strongly Coupled Plasmons of Nanoparticle Dimers. Nano Lett. 2009, 9, 36193625 .

(23) Jin, R.; Cao, Y.; Mirkin, C. A.; Kelly, K. L.; Schatz, G. C.; Zheng, J. G. Photoinduced Conversion of Silver Nanospheres to Nanoprisms. Science. 2001, 294, 1901-1903.

(24) Haynes, C.; Duyne, R. Van. Nanosphere Lithography: A Versatile Nanofabrication Tool for Studies of Size-Dependent Nanoparticle Optics. J. Phys. Chem. B 2001, 105, 55995611.

(25) Quinten, M.; Kreibig, U.; Schönauer, D.; Genzel, L. Optical Absorption Spectra of Pairs of Small Metal Particles. Surf. Sci. 1985, 156, 741-750. 
(26) Aravind, P.; Nitzan, A.; Metiu, H. The Interaction between Electromagnetic Resonances and Its Role in Spectroscopic Studies of Molecules Adsorbed on Colloidal Particles or Metal Spheres. Surf. Sci. 1981, 110, 189-204.

(27) Mirkin, C. A.; Letsinger, R. L.; Mucic, R. C.; Storhoff, J. J. A DNA-Based Method for Rationally Assembling Nanoparticles into Macroscopic Materials. Nature 1996, 382, 607609.

(28) Wang, Z.; Ma, L. Gold Nanoparticle Probes. Coord. Chem. Rev. 2009, 253, 1607-1618.

(29) Sönnichsen, C.; Reinhard, B. M.; Liphardt, J.; Alivisatos, a P. A Molecular Ruler Based on Plasmon Coupling of Single Gold and Silver Nanoparticles. Nat. Biotechnol. 2005, 23, $741-745$.

(30) Sweatlock, L.; Maier, S.; Atwater, H.; Penninkhof, J.; Polman, a. Highly Confined Electromagnetic Fields in Arrays of Strongly Coupled Ag Nanoparticles. Phys. Rev. B 2005, 71, 235408.

(31) Halas, N. J.; Lal, S.; Chang, W.-S.; Link, S.; Nordlander, P. Plasmons in Strongly Coupled Metallic Nanostructures. Chem. Rev. 2011, 111, 3913-3961.

(32) Nordlander, P.; Oubre, C.; Prodan, E.; Li, K.; Stockman, M. I. Plasmon Hybridization in Nanoparticle Dimers. Nano Lett. 2004, 4, 899-903.

(33) Prodan, E.; Radloff, C.; Halas, N. J.; Nordlander, P. A Hybridization Model for the Plasmon Response of Complex Nanostructures. Science. 2003, 302, 419-422.

(34) Moskovits, M. Surface-Enhanced Raman Spectroscopy: A Brief Retrospective. J. Raman Spectrosc. 2005, 36, 485-496.

(35) Marhaba, S.; Bachelier, G.; Bonnet, C.; Broyer, M.; Cottancin, E.; Grillet, N.; Lermé, J.; Vialle, J.-L.; Pellarin, M. Surface Plasmon Resonance of Single Gold Nanodimers near the Conductive Contact Limit. J. Phys. Chem. C 2009, 113, 4349-4356.

(36) Romero, I.; Aizpurua, J.; Bryant, G. W.; García De Abajo, F. J. Plasmons in Nearly Touching Metallic Nanoparticles: Singular Response in the Limit of Touching Dimers. Opt. Express 2006, 14, 9988-9999.

(37) Lassiter, J. B.; Aizpurua, J.; Hernandez, L. I.; Brandl, D. W.; Romero, I.; Lal, S.; Hafner, J. H.; Nordlander, P.; Halas, N. J. Close Encounters between Two Nanoshells 2008. Nano Lett. 2008, 8, 1212-1218.

(38) Scholl, J. a; García-etxarri, A.; Koh, A. L.; Dionne, J. A. Observation of Quantum Tunneling between Two Plasmonic Nanoparticles. Nano Lett. 2013, 13, 564-569. 
(39) Esteban, R.; Borisov, A. G.; Nordlander, P.; Aizpurua, J. Bridging Quantum and Classical Plasmonics with a Quantum-Corrected Model. Nat. Commun. 2012, 3, 825.

(40) Zuloaga, J.; Prodan, E.; Nordlander, P. Quantum Description of the Plasmon Resonances of a Nanoparticle Dimer. Nano Lett. 2009, 9, 887-891.

(41) Savage, K. J.; Hawkeye, M. M.; Esteban, R.; Borisov, A. G.; Aizpurua, J.; Baumberg, J. J. Revealing the Quantum Regime in Tunnelling Plasmonics. Nature 2012, 491, 574-577.

(42) Esteban, R.; Borisov, A. G.; Nordlander, P.; Aizpurua, J. Bridging Quantum and Classical Plasmonics with a Quantum-Corrected Model. Nat. Commun. 2012, 3, 825-829.

(43) Su, K.; Wei, Q.; Zhang, X.; Mock, J. J.; Smith, D. R.; Schultz, S. Interparticle Coupling Effects on Plasmon Resonances of Nanogold Particles. Nano Lett. 2003, 3, 1086-1090.

(44) Rockstuhl, C.; Salt, M. G.; Herzig, H. P. Analyzing the Scattering Properties of Coupled Metallic Nanoparticles. J. Opt. Soc. Am. A 2004, 21, 1761-1768.

(45) Mao, L.; Li, Z.; Wu, B.; Xu, H. Effects of Quantum Tunneling in Metal Nano-Gap on Surface-Enhanced Raman Scattering. Appl. Phys. Lett. 2009, 94, 243102.

(46) Wu, Y.; Nordlander, P. Finite-Difference Time-Domain Modeling of the Optical Properties of Nanoparticles near Dielectric Substrates. J. Phys. Chem. C 2010, 114, 73027307.

(47) Nordlander, P.; Prodan, E. Plasmon Hybridization in Nanoparticles near Metallic Surfaces. Nano Lett. 2004, 4, 2209-2213.

(48) Halas, N. J.; Lal, S.; Chang, W.-S.; Link, S.; Nordlander, P. Plasmons in Strongly Coupled Metallic Nanostructures. Chem. Rev. 2011, 111, 3913-3961.

(49) Ringe, E.; McMahon, J. M.; Sohn, K.; Cobley, C.; Xia, Y.; Huang, J.; Schatz, G. C.; Marks, L. D.; Van Duyne, R. P. Unraveling the Effects of Size, Composition, and Substrate on the Localized Surface Plasmon Resonance Frequencies of Gold and Silver Nanocubes: A Systematic Single-Particle Approach. J. Phys. Chem. C 2010, 114, 1251112516.

(50) Ahamad, N.; Bottomley, A.; Ianoul, A. Optimizing Refractive Index Sensitivity of Supported Silver Nanocube Monolayers. J. Phys. Chem. C 2012, 116, 185-192.

(51) Bottomley, A.; Prezgot, D.; Staff, A.; Ianoul, A. Fine Tuning of Plasmonic Properties of Monolayers of Weakly Interacting Silver Nanocubes on Thin Silicon Films. Nanoscale 2012, 4, 6374-6382. 
(52) König, T.; Kodiyath, R.; Combs, Z. a.; Mahmoud, M. a.; El-Sayed, M. a.; Tsukruk, V. V. Silver Nanocube Aggregates in Cylindrical Pores for Higher Refractive Index Plasmonic Sensing. Part. Part. Syst. Charact. 2014, 31, 274-283.

(53) Zhang, L.; Niu, W.; Xu, G. Synthesis and Applications of Noble Metal Nanocrystals with High-Energy Facets. Nano Today 2012, 7, 586-605.

(54) Lee, C.; Meisel, D. Adsorption and Surface-Enhanced Raman of Dyes on Silver and Gold Sols'. J. Phys. Chem. 1982, 86, 3391-3395.

(55) Pillai, Z. S.; Kamat, P. V. What Factors Control the Size and Shape of Silver Nanoparticles in the Citrate Ion Reduction Method? J. Phys. Chem. B 2004, 108, 945951.

(56) Peng, S.; McMahon, J. M.; Schatz, G. C.; Gray, S. K.; Sun, Y. Reversing the SizeDependence of Surface Plasmon Resonances. Proc. Natl. Acad. Sci. U. S. A. 2010, 107, 14530-14534.

(57) Chen, M.; Feng, Y.-G.; Wang, X.; Li, T.-C.; Zhang, J.-Y.; Qian, D.-J. Silver Nanoparticles Capped by Oleylamine: Formation, Growth, and Self-Organization. Langmuir 2007, 23, 5296-5304.

(58) Skrabalak, S. E.; Wiley, B. J.; Kim, M.; Formo, E. V; Xia, Y. On the Polyol Synthesis of Silver Nanostructures: Glycolaldehyde as a Reducing Agent. Nano Lett. 2008, 8, $2077-$ 2081.

(59) LaMer, V. K.; Dinegar, R. H. Theory, Production and Mechanism of Formation of Monodispersed Hydrosols. J. Am. Chem. Soc. 1950, 72, 4847-4854.

(60) Sun, Y. Controlled Synthesis of Colloidal Silver Nanoparticles in Organic Solutions: Empirical Rules for Nucleation Engineering. Chem. Soc. Rev. 2013, 42, 2497-2511.

(61) Iijima, S.; Ichihashi, T. Structural Instability of Ultrafine Particles of Metals. Phys. Rev. Lett. 1986, 56, 616-619.

(62) Wiley, B.; Sun, Y.; Xia, Y. Synthesis of Silver Nanostructures with Controlled Shapes and Properties. Acc. Chem. Res. 2007, 40, 1067-1076.

(63) Zeng, J.; Zheng, Y.; Rycenga, M.; Tao, J. Controlling the Shapes of Silver Nanocrystals with Different Capping Agents. J. Am. Chem. Soc. 2010, 132, 8552-8553.

(64) Al-Saidi, W. a; Feng, H.; Fichthorn, K. a. Adsorption of Polyvinylpyrrolidone on Ag Surfaces: Insight into a Structure-Directing Agent. Nano Lett. 2012, 12, 997-1001. 
(65) Kilin, D. S.; Prezhdo, O. V.; Xia, Y. Shape-Controlled Synthesis of Silver Nanoparticles: Ab Initio Study of Preferential Surface Coordination with Citric Acid. Chem. Phys. Lett. 2008, 458, 113-116.

(66) Sun, Y.; Xia, Y. Shape-Controlled Synthesis of Gold and Silver Nanoparticles. Science (80-. ). 2002, 298, 2176-2179.

(67) Zhang, Q.; Moran, C. H.; Xia, X.; Rycenga, M.; Li, N.; Xia, Y. Synthesis of Ag Nanobars in the Presence of Single-Crystal Seeds and a Bromide Compound, and Their SurfaceEnhanced Raman Scattering (SERS) Properties. Langmuir 2012, 28, 9047-9054.

(68) Korte, K. E.; Skrabalak, S. E.; Xia, Y. Rapid Synthesis of Silver Nanowires through a $\mathrm{CuCl}-$ or CuCl2-Mediated Polyol Process. J. Mater. Chem. 2008, 18, 437.

(69) Sun, Y.; Mayers, B.; Herricks, T.; Xia, Y. Polyol Synthesis of Uniform Silver Nanowires: A Plausible Growth Mechanism and the Supporting Evidence. Nano Lett. 2003, 3, 955-960.

(70) Wiley, B.; Herricks, T.; Sun, Y.; Xia, Y. Polyol Synthesis of Silver Nanoparticles: Use of Chloride and Oxygen to Promote the Formation of Single-Crystal, Truncated Cubes and Tetrahedrons. Nano Lett. 2004, 4, 1733-1739.

(71) Wiley, B. J.; Xiong, Y.; Li, Z.-Y.; Yin, Y.; Xia, Y. Right Bipyramids of Silver: A New Shape Derived from Single Twinned Seeds. Nano Lett. 2006, 6, 765-768.

(72) Siekkinen, A. R.; McLellan, J. M.; Chen, J.; Xia, Y. Rapid Synthesis of Small Silver Nanocubes by Mediating Polyol Reduction with a Trace Amount of Sodium Sulfide or Sodium Hydrosulfide. Chem. Phys. Lett. 2006, 432, 491-496.

(73) Kryukov, a. I.; Stroyuk, a. L.; Zin'chuk, N. N.; Korzhak, a. V.; Kuchmii, S. Y. Optical and Catalytic Properties of Ag2S Nanoparticles. J. Mol. Catal. A Chem. 2004, 221, 209-221.

(74) Xiong, Y.; Washio, I.; Chen, J.; Cai, H.; Li, Z.-Y.; Xia, Y. Poly(vinyl Pyrrolidone): A Dual Functional Reductant and Stabilizer for the Facile Synthesis of Noble Metal Nanoplates in Aqueous Solutions. Langmuir 2006, 22, 8563-8570.

(75) Zhang, Q.; Li, W.; Moran, C.; Zeng, J.; Chen, J.; Wen, L.-P.; Xia, Y. Seed-Mediated Synthesis of Ag Nanocubes with Controllable Edge Lengths in the Range of 30-200 Nm and Comparison of Their Optical Properties. J. Am. Chem. Soc. 2010, 132, 11372-11378.

(76) Xia, X.; Zeng, J.; Oetjen, L. K.; Li, Q.; Xia, Y. Quantitative Analysis of the Role Played by Poly(vinylpyrrolidone) in Seed-Mediated Growth of Ag Nanocrystals. J. Am. Chem. Soc. 2012, 134, 1793-1801.

(77) Kuo, C. L.; Hwang, K. C. Does Morphology of a Metal Nanoparticle Play a Role in Ostwald. Chem. Mater. 2013, 25, 365-371. 
(78) Cheng, L.; Song, J.; Yin, J.; Duan, H. Self-Assembled Plasmonic Dimers of Amphiphilic Gold Nanocrystals. J. Phys. Chem. Lett. 2011, 2, 2258-2262.

(79) Xia, H.; Su, G.; Wang, D. Size-Dependent Electrostatic Chain Growth of pH-Sensitive Hairy Nanoparticles. Angew. Chem. Int. Ed. Engl. 2013, 52, 3726-3730.

(80) Toma, M.; Toma, K.; Michioka, K.; Ikezoe, Y.; Obara, D.; Okamoto, K.; Tamada, K. Collective Plasmon Modes Excited on a Silver Nanoparticle 2D Crystalline Sheet. Phys. Chem. Chem. Phys. 2011, 13, 7459-7466.

(81) Henzie, J.; Grünwald, M.; Widmer-Cooper, A.; Geissler, P. L.; Yang, P. Self-Assembly of Uniform Polyhedral Silver Nanocrystals into Densest Packings and Exotic Superlattices. Nat. Mater. 2012, 11, 131-137.

(82) Gong, J.; Li, G.; Tang, Z. Self-Assembly of Noble Metal Nanocrystals : Fabrication, Optical Property, and Application. Nano Today 2012, 7, 564-585.

(83) El Badawy, A. M.; Luxton, T. P.; Silva, R. G.; Scheckel, K. G.; Suidan, M. T.; Tolaymat, T. M. Impact of Environmental Conditions (pH, Ionic Strength, and Electrolyte Type) on the Surface Charge and Aggregation of Silver Nanoparticles Suspensions. Environ. Sci. Technol. 2010, 44, 1260-1266.

(84) Walker, D. a; Kowalczyk, B.; de la Cruz, M. O.; Grzybowski, B. a. Electrostatics at the Nanoscale. Nanoscale 2011, 3, 1316-1344.

(85) Klinkova, A.; Choueiri, R. M.; Kumacheva, E. Self-Assembled Plasmonic Nanostructures. Chem. Soc. Rev. 2014, 43, 3976-3991.

(86) Love, J. C.; Estroff, L. a; Kriebel, J. K.; Nuzzo, R. G.; Whitesides, G. M. Self-Assembled Monolayers of Thiolates on Metals as a Form of Nanotechnology. Chem. Rev. 2005, 105, $1103-1169$.

(87) Chen, C.-F.; Tzeng, S.-D.; Chen, H.-Y.; Lin, K.-J.; Gwo, S. Tunable Plasmonic Response from Alkanethiolate-Stabilized Gold Nanoparticle Superlattices: Evidence of near-Field Coupling. J. Am. Chem. Soc. 2008, 130, 824-826.

(88) Wei, A. Calixarene-Encapsulated Nanoparticles: Self-Assembly into Functional Nanomaterials. Chem. Commun. 2006, 1581-1591.

(89) Rycenga, M.; McLellan, J. M.; Xia, Y. Controlling the Assembly of Silver Nanocubes through Selective Functionalization of Their Faces. Adv. Mater. 2008, 20, 2416-2420.

(90) Martin-Gassin, G.; El Harfouch, Y.; Benichou, E.; Bachelier, G.; Russier-Antoine, I.; Jonin, C.; Roux, S.; Tillement, O.; Brevet, P.-F. Correlation Reflectance Spectroscopy of Heterogeneous Silver Nanoparticle Films upon Compression at the Air/water Interface. $J$. Phys. Condens. Matter 2008, 20, 055228. 
(91) Tanoue, Y.; Sugawa, K.; Yamamuro, T.; Akiyama, T. Densely Arranged TwoDimensional Silver Nanoparticle Assemblies with Optical Uniformity over Vast Areas as Excellent Surface-Enhanced Raman Scattering Substrates. Phys. Chem. Chem. Phys. 2013, 15, 15802-15805.

(92) Acharya, S.; Hill, J. P.; Ariga, K. Soft Langmuir-Blodgett Technique for Hard Nanomaterials. Adv. Mater. 2009, 21, 2959-2981.

(93) Mahmoud, M. a.; Tabor, C. E.; El-Sayed, M. a. Surface-Enhanced Raman Scattering Enhancement by Aggregated Silver Nanocube Monolayers Assembled by the Langmuir-Blodgett Technique at Different Surface Pressures. J. Phys. Chem. C 2009, $113,5493-5501$.

(94) Mahmoud, M. a.; El-Sayed, M. a. Comparative Study of the Assemblies and the Resulting Plasmon Fields of Langmuir-Blodgett Assembled Monolayers of Silver Nanocubes and Gold Nanocages. J. Phys. Chem. C 2008, 112, 14618-14625.

(95) Shin, H.; Kim, H.; Ha, J.; Lim, K.-S.; Lee, M. Langmuir-Blodgett Ag Nanoparticle Monolayer Patterned by Pulsed Laser-Induced Selective Desorption. Superlattices Microstruct. 2008, 44, 657-663.

(96) Ahamad, N.; Prezgot, D.; Ianoul, A. Patterning Silver Nanocubes in Monolayers Using Phase Separated Lipids as Templates. J. Nanoparticle Res. 2012, 14, 724.

(97) Moraille, P.; Badia, A. Highly Parallel, Nanoscale Stripe Morphology in Mixed Phospholipid Monolayers Formed by Langmuir-Blodgett Transfer. Langmuir 2002, 18 , 4414-4419.

(98) Chen, C. K.; De Castro, A. R. B.; Shen, Y. R. Surface-Enhanced Second-Harmonic Generation. Phys. Rev. Lett. 1981, 46, 145-148.

(99) Hartstein, A.; Kirtley, J.; Tsang, J. Enhancement of the Infrared Absorption from Molecular Monolayers with Thin Metal Overlayers. Phys. Rev. Lett. 1980, 45, 201-204.

(100) Guerrero, A. R.; Aroca, R. F. Surface-Enhanced Fluorescence with Shell-Isolated Nanoparticles (SHINEF). Angew. Chemie Int. Ed. 2011, 50, 665-668.

(101) Fleischmann, M.; Hendra, P.; McQuillan, A. Raman Spectra of Pyridine Adsorbed at a Silver Electrode. Chem. Phys. Lett. 1974, 26, 2-5.

(102) Tian, Z.; Ren, B.; Wu, D. Surface-Enhanced Raman Scattering : From Noble to Transition Metals and from Rough. J. Phys. Chem. B 2002, 106, 9463-9483.

(103) Jeanmaire, D.; Duyne, R. Van. Surface Raman Spectroelectrochemistry: Part I. Heterocyclic, Aromatic, and Aliphatic Amines Adsorbed on the Anodized Silver Electrode. J. Electroanal. Chem. 1977, 84, 1-20. 
(104) Nie, S. Probing Single Molecules and Single Nanoparticles by Surface-Enhanced Raman Scattering. Science. 1997, 275, 1102-1106.

(105) Kneipp, K.; Wang, Y.; Kneipp, H.; Perelman, L.; Itzkan, I.; Dasari, R.; Feld, M. Single Molecule Detection Using Surface-Enhanced Raman Scattering (SERS). Phys. Rev. Lett. 1997, 78, 1667-1670.

(106) Moskovits, M. Surface-Enhanced Spectroscopy. Rev. Mod. Phys. 1985, 57, 783-826.

(107) Blackie, E. J.; Le Ru, E. C.; Etchegoin, P. G. Single-Molecule Surface-Enhanced Raman Spectroscopy of Nonresonant Molecules. J. Am. Chem. Soc. 2009, 131, 14466-14472.

(108) McLean, T. M.; Cleland, D.; Gordon, K. C.; Telfer, S. G.; Waterland, M. R. Raman Spectroscopy of Dipyrrins: Nonresonant, Resonant and Surface-Enhanced Cross-Sections and Enhancement Factors. J. Raman Spectrosc. 2011, 42, 2154-2164.

(109) Maher, R. C.; Cohen, L. F.; Le Ru, E. C.; Etchegoin, P. G. On the Experimental Estimation of Surface Enhanced Raman Scattering (SERS) Cross Sections by Vibrational Pumping. J. Phys. Chem. B 2006, 110, 19469-19478.

(110) Le Ru, E. C.; Blackie, E.; Meyer, M.; Etchegoin, P. G.; Ru, E. C. Le. Surface Enhanced Raman Scattering Enhancement Factors: A Comprehensive Study. J. Phys. Chem. C 2007, 111, 13794-13803.

(111) Moskovits, M. Surface-Enhanced Raman Spectroscopy: A Brief Retrospective. J. Raman Spectrosc. 2005, 36, 485-496.

(112) McCreery, R. L. Raman Spectroscopy for Chemical Analysis; John Wiley \& Sons, Inc.: Hoboken, NJ, USA, 2000.

(113) Watanabe, T.; Yanagihara, N.; Honda, K.; Pettinger, B.; Moerl, L. Effects of Underpotentially Deposited $\mathrm{Tl}$ and $\mathrm{Pb}$ Submonolayers on the Surface-Enhanced Raman Scattering (SERS) from Pyridine at Ag Electrodes. Chem. Phys. Lett. 1983, 96, 649-655.

(114) Kneipp, K.; Wang, Y.; Kneipp, H.; Itzkan, I.; Dasari, R.; Feld, M. Population Pumping of Excited Vibrational States by Spontaneous Surface-Enhanced Raman Scattering. Phys. Rev. Lett. 1996, 76, 2444-2447.

(115) Tsai, D. P.; Kovacs, J.; Wang, Z.; Moskovits, M.; Shalaev, V. M.; Suh, J. S.; Botet, R. Photon Scanning Tunneling Microscopy Images of Optical Excitations of Fractal Metal Colloid Clusters. Phys. Rev. Lett. 1994, 72, 4149-4153.

(116) Zhang, P.; Haslett, T.; Douketis, C.; Moskovits, M. Mode Localization in Self-Affine Fractal Interfaces Observed by near-Field Microscopy. Phys. Rev. B 1998, 57, 1551315518. 
(117) Markel, V.; Shalaev, V.; Zhang, P.; Huynh, W.; Tay, L.; Haslett, T.; Moskovits, M. NearField Optical Spectroscopy of Individual Surface-Plasmon Modes in Colloid Clusters. Phys. Rev. B 1999, 59, 10903-10909.

(118) Xu, H.; Aizpurua, J.; Kall, M.; Apell, P. Electromagnetic Contributions to SingleMolecule Sensitivity in Surface-Enhanced Raman Scattering. Phys. Rev. E 2000, 62, $4318-4324$.

(119) Fang, Y.; Seong, N.-H.; Dlott, D. D. Measurements of the Distribution of Site Enhancements in Surface-Enhanced Raman Scattering. Science. 2008, 321, 388-393.

(120) Nie, S. Probing Single Molecules and Single Nanoparticles by Surface-Enhanced Raman Scattering. Science. 1997, 275, 1102-1106.

(121) Le Ru, E. C.; Etchegoin, P. G.; Meyer, M. Enhancement Factor Distribution around a Single Surface-Enhanced Raman Scattering Hot Spot and Its Relation to Single Molecule Detection. J. Chem. Phys. 2006, 125, 204701.

(122) Moskovits, M. Persistent Misconceptions Regarding SERS. Phys. Chem. Chem. Phys. 2013, 15, 5301-5311.

(123) Lombardi, J. R.; Birke, R. L. Time-Dependent Picture of the Charge-Transfer Contributions to Surface Enhanced Raman Spectroscopy. J. Chem. Phys. 2007, 126, 244709 .

(124) Krasser, W.; Renouprez, A. Enhanced Raman Scattering of Benzene Chemisorbed on Small Platinum Clusters. Solid State Commun. 1982, 41, 231-235.

(125) Demuth, J.; Sanda, P. Observation of Charge-Transfer States for Pyridine Chemisorbed on Ag (111). Phys. Rev. Lett. 1981, 47, 57.

(126) Adrian, F. J. Charge Transfer Effects in Surface-Enhanced Raman Scatteringa). J. Chem. Phys. 1982, 77, 5302.

(127) Le Ru, E. C.; Schroeter, L. C.; Etchegoin, P. G. Direct Measurement of Resonance Raman Spectra and Cross Sections by a Polarization Difference Technique. Anal. Chem. 2012, $84,5074-5079$.

(128) Chance, R. R. Lifetime of an Emitting Molecule near a Partially Reflecting Surface. $J$. Chem. Phys. 1974, 60, 2744.

(129) Prezgot, D.; Ianoul, A. Optical Properties of Strongly Interacting Supported Silver Nanocube Monolayers. Proc. SPIE 2013, 8597, 85970A.

(130) Skrabalak, S. E.; Au, L.; Li, X.; Xia, Y. Facile Synthesis of Ag Nanocubes and Au Nanocages. Nat. Protoc. 2007, 2, 2182-2190. 
(131) Wang, Y.; Li, Z.; Zhao, K.; Sobhani, A.; Zhu, X.; Fang, Z.; Halas, N. J. SubstrateMediated Charge Transfer Plasmons in Simple and Complex Nanoparticle Clusters. Nanoscale 2013, 5, 9897-9901.

(132) Moran, C.; Rycenga, M. Replacement of Poly (vinyl Pyrrolidone) by Thiols: A Systematic Study of Ag Nanocube Functionalization by Surface-Enhanced Raman Scattering. J. Phys. ... 2011, 115, 21852-21857.

(133) Mahmoud, M. a.; Tabor, C. E.; El-Sayed, M. a. Surface-Enhanced Raman Scattering Enhancement by Aggregated Silver Nanocube Monolayers Assembled by the Langmuir-Blodgett Technique at Different Surface Pressures. J. Phys. Chem. C 2009, $113,5493-5501$.

(134) Wang, Y.; Li, Z.; Zhao, K.; Sobhani, A.; Zhu, X. Substrate-Mediated Charge Transfer Plasmons in Simple and Complex Nanoparticle Clusters. Nanoscale 2013, 5, 9897-9901.

(135) Tay, L.-L.; Hulse, J. Surface-Enhanced Raman and Optical Scattering in Coupled Plasmonic Nanoclusters. J. Mod. Opt. 2013, 60, 1107-1114.

(136) Yin, J.; Yue, C.; Zang, Y.; Chiu, C.-H.; Li, J.; Kuo, H.-C.; Wu, Z.; Li, J.; Fang, Y.; Chen, C. Effect of the Surface-Plasmon-Exciton Coupling and Charge Transfer Process on the Photoluminescence of Metal-Semiconductor Nanostructures. Nanoscale 2013, 5, 44364442 .

(137) Ben, X.; Cao, P.; Park, H. The Effect of Planar Defects on the Optical Properties of Silver Nanostructures. J. Phys. Chem. C 2013, 117, 13738-13746.

(138) Xia, X.; Xie, S.; Liu, M.; Peng, H.-C.; Lu, N.; Wang, J.; Kim, M. J.; Xia, Y. On the Role of Surface Diffusion in Determining the Shape or Morphology of Noble-Metal Nanocrystals. Proc. Natl. Acad. Sci. U. S. A. 2013, 110, 6669-6673.

(139) Wei, A. Calixarene-Encapsulated Nanoparticles: Self-Assembly into Functional Nanomaterials. Chem. Commun. (Camb). 2006, 1581-1591.

(140) Pedersen, D. B.; Wang, S. Real-Time Experiments on the Spontaneous Restructuring of Self-Assemblies of Naked Ag Nanoparticle Anions: How Close Can Two Nanoparticles Get? J. Phys. Chem. C 2012, 116, 3258-3265.

(141) Moran, C. H.; Rycenga, M.; Zhang, Q.; Xia, Y. Replacement of Poly ( Vinyl Pyrrolidone ) by Thiols: A Systematic Study of Ag Nanocube Functionalization by Surface-Enhanced Raman Scattering. J. Phys. Chem. C 2011, 115, 21852-21857.

(142) Schoenfisch, M. H.; Pemberton, J. E. Air Stability of Alkanethiol Self-Assembled Monolayers on Silver and Gold Surfaces. J. Am. Chem. Soc. 1998, 120, 4502-4513. 
(143) McCreery, R. L. Photometric Standards for Raman Spectroscopy Photometric Standards for Raman Spectroscopy. In Handbook of Vibrational Spectroscopy; John Wiley \& Sons, Ltd, 2002.

(144) Whelan, C. M.; Smyth, M. R.; Barnes, C. J. HREELS, XPS, and Electrochemical Study of Benzenethiol Adsorption on Au(111). Langmuir 1999, 15, 116-126.

(145) McFarland, A. D.; Young, M. a; Dieringer, J. a; Van Duyne, R. P. Wavelength-Scanned Surface-Enhanced Raman Excitation Spectroscopy. J. Phys. Chem. B 2005, 109, 1127911285 .

(146) Wan, L.-J.; Terashima, M.; Noda, H.; Osawa, M. Molecular Orientation and Ordered Structure of Benzenethiol Adsorbed on Gold(111). J. Phys. Chem. B 2000, 104, 35633569.

(147) Whitney, A. V; Elam, J. W.; Zou, S.; Zinovev, A. V; Stair, P. C.; Schatz, G. C.; Van Duyne, R. P. Localized Surface Plasmon Resonance Nanosensor: A High-Resolution Distance-Dependence Study Using Atomic Layer Deposition. J. Phys. Chem. B 2005, 109, 20522-20528.

(148) Tay, L.-L.; Hulse, J. Surface-Enhanced Raman and Optical Scattering in Coupled Plasmonic Nanoclusters. J. Mod. Opt. 2013, 60, 1107-1114.

(149) Hooshmand, N.; Bordley, J.; El-Sayed, M. Are Hot Spots between Two Plasmonic Nanocubes of Silver or Gold Formed between Adjacent Corners or Facets? A DDA Examination. J. Phys. Chem. Lett. 2014, 5, 2229-2234.

(150) Camargo, P. H. C.; Rycenga, M.; Au, L.; Xia, Y. Isolating and Probing the Hot Spot Formed between Two Silver Nanocubes. Angew. Chemie 2009, 121, 2214-2218.

(151) Tan, S.; Wu, L.; Yang, J.; Bai, P.; Bosman, M.; Nijhuis, C. Quantum Plasmon Resonances Controlled by Molecular Tunnel Junctions. Science. 2014, 343, 1496.

(152) Doherty, M. D.; Murphy, A.; McPhillips, J.; Pollard, R. J.; Dawson, P. Wavelength Dependence of Raman Enhancement from Gold Nanorod Arrays: Quantitative Experiment and Modeling of a Hot Spot Dominated System. J. Phys. Chem. C 2010, 114, 19913-19919.

(153) Herzog, J. B.; Knight, M. W.; Li, Y.; Evans, K. M.; Halas, N. J.; Natelson, D. Dark Plasmons in Hot Spot Generation and Polarization in Interelectrode Nanoscale Junctions. Nano Lett. 2013, 13, 1359-1364. 